\title{
IMERSÕES SUB-RIEMANNIANAS EM \\ FORMAS ESPACIAIS COMPLEXAS
}

\author{
Albetã Costa Mafra
}

\author{
DISSERTAÇÃO APRESENTADA \\ $\mathrm{AO}$ \\ INSTITUTO DE MATEMÁTICA E ESTATÍSTICA \\ DA \\ UNIVERSIDADE DE SÃO PAULO \\ PARA \\ OBTENÇÃO DO GRAU \\ $\mathrm{DE}$ \\ MESTRE EM MATEMÁTICA \\ Área de concentração: Geometria Diferencial \\ Orientador: Prof. Dr. José Antonio Verderesi
}

São Paulo, Fevereiro de 2001 


\section{IMERSÕES SUB-RIEMANNIANAS \\ EM \\ FORMAS ESPACIAIS COMPLEXAS}

Este trabalho corresponde à redação final da dissertação devidamente corrigida

e apresentada por Albetã Costa Mafra e aprovada pela Comissão Julgadora.

São Paulo, 05 de Maio de 2001

Banca examinadora:

Prof. Dr José Antônio Verderesi (Orientador) - IME-USP

Prof. Dr Renato Pedrosa - IMECC-UNICAMP

Prof. Dr Antônio Carlos Asperti - IME-USP 


\section{Agradecimentos}

- ao Prof. José Antonio Verderesi pela paciência e seriedade durante os quatro anos de orientação acadêmica: 2 de iniciação ciêntifica e 2 de mestrado em matematica.

- ao Verderesi, amigo.

- ao Prof. e amigo Carlos Alberto Knudsen pelos incentivos diretos e indiretos.

- aos amigos do CRUSP, em especial aos que moraram comigo e ao apartamento B511.

- ao amigo Vilhena.

- à Prof $\underline{a}$. Leilá Figueiredo, de quem uma pergunta me fez mudar a forma de pensar.

- em especial àqueles aos quais mais amo neste mundo: meus pais. 


\section{Resumo}

O objetivo deste trabalho é estudar teoremas de rigidez para um tipo especial de variedades sub-Riemannianas isometricamente imersas em um espaço de formas complexas.

Definimos e estudamos algumas características de um tipo especial de geometria subRiemanniana. Provamos a existência e unicidade de uma conexão associada à estrutura sub-Riemanniana e a relacionamos com uma determinada conexão de Levi-Civita.

Após uma breve revisão da teoria de uma hipervariedade isometricamanete imersa em um variedade Riemanniana e do estudo de um caso particular de submersão Riemanniana, provamos alguns teoremas de rigidez para uma hipervariedade sub-Riemanniana isometricamente imersa em uma forma espacial complexa. Finalizamos analisando o caso de imersões de variedades sub-Riemannianas homogêneas tridimensionais e fazemos alguns exemplos de imersões isométricas. 


\section{Abstract}

The purpose of this work is to study rigidity theorems for a special type of subRiemannian hypersurfaces that are isometricaly immersed in complex space forms.

We define and study a special type of sub-Riemannian manifold. We prove the existence and uniqueness of a connection associated with that sub-Riemannian structure and we also relate it to the Levi-Civita connection of a metric which is naturally defined from the sub-Riemannian one.

After doing a brief review of the theory of isometric immersions of hypersurfaces in Riemannian manifolds and examining a special type of Riemannian submersion we prove theorems related to the rigidity of isometric immersions of sub-Riemannian hypersurfaces in complex space forms. We finish this work studying the tridimensional homogeneous sub-Riemannian case and showing some examples of isometric immersions in complex space forms. 


\section{Índice}

$\begin{array}{ll}\text { Agradecimentos } & \text { iii }\end{array}$

Resumo $\quad$ iv

Abstract $\quad$ v

$\begin{array}{ll}\text { Introdução } & 1\end{array}$

1 Espaços de curvatura holomorfa constante 4

1.1 Espaços vetorias quase-complexos e variedades complexas . . . . . . . . . 4

1.2 O espaço projetivo complexo . . . . . . . . . . . . . . . . 11

1.3 O espaço hiperbólico complexo . . . . . . . . . . . . . . . 18

2 Geometria sub-Riemanniana 20

2.1 Definições e a conexão sub-Riemanniana . . . . . . . . . . . . . . . 20

2.2 Propriedades da conexão sub-Riemanniana . . . . . . . . . . . . . . . 23

2.3 Exemplos de variedades sub-Riemannianas homogêneas . . . . . . . . . 25

2.4 Relação entre as conexões Riemanniana e sub-Riemanniana . . . . . . . . . . 34

3 Imersões Isométricas sub-Riemannianas 36

3.1 Hipervariedades . . . . . . . . . . . . . . . . . . . . 36

3.2 Submersões . . . . . . . . . . . . . . . . . . . . . . . . 38

3.3 O caso sub-Riemanniano . . . . . . . . . . . . . . . . 46 
3.4 O caso sub-Riemanniano tridimensional . . . . . . . . . . . . . . . . . 60

3.5 Imersões isométricas de $S_{r}^{3}$ em $N(c) \ldots \ldots \ldots \ldots$

3.6 Imersão isométrica de $Q_{r}^{3}$ em $N(c) \quad \ldots \ldots \ldots$. . . . . . . . . . 75

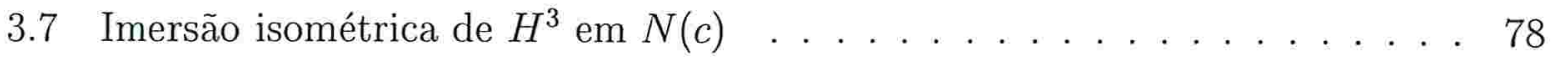




\section{Introdução}

Geometria sub-Riemanniana pode ser entendida como uma generalização do que é classicamente conhecido como geometria Riemanniana. Em resumo, geometria sub-Riemanniana é a geometria de uma distribuição métrica, isto é, a geometria de uma variedade diferenciável $M$ a qual associamos um sub-fibrado $\mathcal{D}$, do fibrado tangente $T M$, o qual esta munido com uma métrica $g$. Este tipo de estrutura $(M, \mathcal{D}, g)$ tem aparecido nos mais diferentes ramos da matemática pura e aplicada. $\mathrm{O}$ caso $\mathcal{D}=T M$ resgata a geometria Riemanniana enquanto que $\mathcal{D} \neq T M$ e integrável nos levará a teoria das folheações. Ambos os casos foram e são bastante estudados. Porém, apesar do caso Riemanniano e de folheações, com folhas munidas com uma métrica, poderem ser considerados sub-Riemannianos é mais comum associar o nome sub-Riemanniano ao caso no qual $\mathcal{D}$ é um sub-fibrado não integrável. Alguns casos desta geometria nos quais $\mathcal{D}$ é não integrável são amplamente estudados, podemos citar, entre outros, o caso de conctato, isto é, o caso em que $\mathcal{D}$ tem co-dimensão 1 e a aplicação bilinear

$$
\begin{aligned}
\mathcal{L}: \mathcal{D} \times \mathcal{D} & \longrightarrow T M / \mathcal{D} \\
(X, Y) & \longrightarrow[X, Y] \bmod \mathcal{D}
\end{aligned}
$$

é não degenerada. Neste caso estão associados à distribuição $\mathcal{D}$ uma 1 -forma diferenciável $\theta$ e um campo diferenciável $\xi$ de tal modo que: $\mathcal{D}=\operatorname{ker} \theta,\left(\left.d \theta\right|_{\mathcal{D}}\right)^{n}=2^{n} n ! d V, \theta(\xi)=1$ e $d \theta(\xi,.) \equiv 0$, sendo $d V$ a forma volume em $\mathcal{D}$ e $2 n$ a dimensão de $\mathcal{D}$.

Em nosso trabalho faremos uso de uma estrutura sub-Riemanniana que engloba as de contacto. Tal estrutura é dada por $(M, \mathcal{D}, g, \xi)$, sendo $\xi$ um campo diferenciável, globalmente definido e transversalmente orientável. Este caso é equivalente a tomar um sub-fibrado do fibrado tangente de uma variedade Riemanniana $M$. Em verdade, definindo uma métrica $\tilde{g}$ em $T M$ da seguinte forma: $\left.\tilde{g}\right|_{\mathcal{D} \times \mathcal{D}}=g, \tilde{g}(\xi, \mathcal{D}) \equiv 0$ e $\tilde{g}(\xi, \xi)=1$ transforma- 
se $M$ em uma variedade Riemanniana. Uma forma de se conseguir tais estruturas subRiemannianas é partir de uma varidade $M$ de dimensão $2 n+1$ e imergi-la em uma forma espacial complexa $N(c)$ de curvatura holomorfa $c$ e definir $\mathcal{D}=T M \cap J(T M)$. desta maneira, $\mathcal{D}$ terá dimensão $2 n$ e ficará definida em $\mathcal{D}$ uma estrutura quase-complexa induzida pela estrutura quase-complexa do ambiente $N(c)$. Em essência, o que faremos neste trabalho pode ser relacionado com estruturas de Cauchy-Riemann e variedades Sasakianas [22].

Uma pergunta natural, para geômetras, é: Dadas dadas duas variedades sub-Riemannianas $(M, \mathcal{D}, g, \xi)$ e $(\hat{M}, \widehat{\mathcal{D}}, \hat{g}, \hat{\xi})$ isometricamente imersas em uma forma espacial complexa $N(c)$, em que condições elas diferem por uma isometria complexa $\Psi$ do ambiente $N(c)$, de forma que $\hat{M}=\Psi(M)$ e $\widehat{\mathcal{D}}=\Psi(\mathcal{D})$ ? Para a resposta desta pergunta utilizamos os trabalhos de R. Takagi e outros em [14], [15], [16], [17] e [18] que detém resultados relativos a rigidez de hipervariedades no espaço projetivo complexo. Também fazemos uso dos trabalhos de J. A. Verderesi em [19] e [20] que tratam de teoremas de rigidez em termos dos invariantes sub-Riemannianos.

No primeiro capítulo fazemos algumas definições e teoremas concernentes aos espaços vetoriais quase complexos e às variedades complexas. Também nesse capítulo fazemos toda construção do espaço projetivo complexo $\mathbb{C P}^{n}\left(\frac{4}{r^{2}}\right)$ a partir da esfera de raio $r$. Demonstramos também que certos planos no espaço tangente de $\mathbb{C P}^{n}\left(\frac{4}{r^{2}}\right)$ invariantes pela estrutura quase-complexa possuem as curvaturas seccionais constantes e iguais a $\frac{1}{r^{2}}$. O mesmo cálculo, fazendo algumas pequenas mundanças, leva-nos a verificar que os planos do espaço tangente do espaço hiperbólico complexo $\mathbb{C H}^{n}\left(\frac{4}{r^{2}}\right)$ possuem curvaturas seccionais constantes e iguais a $\frac{-1}{r^{2}}$.

No segundo capítulo definimos o que é uma geometria sub-Riemanniana, enunciamos e demonstramos a existência e unicidade de uma conexão associado à estrutura $(M, \mathcal{D}, g, \xi)$, estudamos algumas de suas propriedades e a relacionamos com a conexão de Levi-Civita de $\tilde{g}$. Nesta parte tambem discutimos o caso de grupos de Lie tridimensionais com métricas invariantes à esquerda.

O terceiro capítulo, o mais relevente acredito, começa com uma breve recordação da teoria de uma imersão isométrica Riemanniana para uma hipervariedade, lá expressamos as equações de Gauss e Mainardi-Codazzi para uma hipervariedade. Como o $\mathbb{C P}^{n}\left(\frac{4}{r^{2}}\right)$ e o $\mathbb{C H}^{n}\left(\frac{4}{r^{2}}\right)$ estão relacionados com espaços de curvaturas seccionais constantes de forma que uma destas relações é um caso particular de submersão Riemanniana, fazemos um pouco da teoria desta submersão Riemanniana com o objetivo de transportar para $N(c)$ 
resultados dos espaços de curvaturas seccionais constante. Tratamos então o problema sub-Riemanniano e reescrevemos as equações de Gauss e Mainardi-Codazzi para este caso, a seguir enunciamos e demonstramos alguns teoremas de rigidez e analisamos o caso de imersões, em $\mathbb{C}^{2}, \mathbb{C P}^{2}\left(\frac{4}{r^{2}}\right)$ e $\mathbb{C H}^{2}\left(\frac{4}{r^{2}}\right)$, de variedades sub-Riemannianas homogêneas tridimensionais. Finalizamos este capítulo com exemplos de algumas imersões . 
CAPÍTUlo 1

\section{Espaços de curvatura holomorfa constante}

Começaremos o nosso trabalho descrevendo os espaços nos quais iremos trabalhar. Estes terão algumas propriedades essenciais para o que pretendemos fazer e serão construidos a partir de variedades diferenciáveis bem conhecidas como o $\mathbb{R}^{2 n}$, a esfera de raio $r\left(S_{r}^{2 n+1}\right)$ e a quádrica $Q_{r}^{2 n+1}$.

\subsection{Espaços vetorias quase-complexos e variedades complexas}

O espaço $\mathbb{C}^{n}$ : Consideremos o espaço Euclidiano $\mathbb{R}^{2 n}$ e $J$ a transformação linear $J$ : $\mathbb{R}^{2 n} \rightarrow \mathbb{R}^{2 n}$ tal que se $\left\{e_{1} ; \ldots ; e_{2 n}\right\}$ é a base canônica usual de $R^{2 n}$ teremos

$$
\begin{array}{rll}
J\left(e_{j}\right)=e_{n+j} & \text { se } & j=1,2, \ldots, n \\
J\left(e_{j}\right)=-e_{j-n} & \text { se } & j=n+1, \ldots, 2 n .
\end{array}
$$

Podemos transformar $\left(\mathbb{R}^{2 n}, J\right)$ em um espaço vetorial sobre $\mathbb{C}$. De fato, se $(a+i b) \in \mathbb{C}$ e $v \in \mathbb{C}^{n}$, definimos $(a+i b) v=a v+b J(v)$. Com esta multiplicação de escalares em $\mathbb{C}$ por vetores em $\mathbb{C}^{n}$ verifica-se que teremos satisfeitos todos os axiomas de espaço vetorial, sendo a soma igual a mesma do $\mathbb{R}^{2 n}$. Ao espaço vetorial sobre $\mathbb{C}$ obtido denotamos por $\mathbb{C}^{n}$. $\mathbb{C}^{n}$ possui uma métrica natural que é a do $\mathbb{R}^{2 N}$, que também pode ser obtida tomando-se a parte real da forma sesquilinear canônica do $\mathbb{C}^{n}$ expressa por $\langle z, w\rangle=z_{1} \bar{w}_{1}+\cdots+z_{n} \bar{w}_{n}$.

Em geral, para um espaço vetorial real $V$ com uma estrutura quase-complexa $J$, isto é, $J: V \rightarrow V$ é linear e $J^{-1}=-J$, podemos fazer o produto tensorial de $V$ por $\mathbb{C}\left(V \otimes_{\mathbb{R}} \mathbb{C}\right.$, 
$\mathbb{C}$ como um $\mathbb{R}$ espaço vetorial) que claramente é um espaço vetorial sobre $\mathbb{C}$. Além disso, $J$ se estende de maneira natural a uma $\tilde{J}: V \otimes_{\mathbb{R}} \mathbb{C} \rightarrow V \otimes_{\mathbb{R}} \mathbb{C}$ tal que

$$
\tilde{J}(u \otimes a)=(J u) \otimes a .
$$

A partir de agora $\otimes=\otimes_{\mathbb{R}}$. Como $\tilde{J}^{2}=-I$, segue que os auto-valores de $\tilde{J}$ são $i$ e $-i$. Se denotarmos

$$
\begin{aligned}
V[i] & =\{u \in V \otimes \mathbb{C} \mid \tilde{J}(u)=i u\}, \\
V[-i] & =\{u \in V \otimes \mathbb{C} \mid \tilde{J}(u)=-i u\}
\end{aligned}
$$

teremos que $V \otimes \mathbb{C}=V[i] \oplus V[-i]$. Um cálculo simples nos mostra que

$$
\begin{aligned}
V[i] & =\{X-i J(X): X \in V\}, \\
V[-i] & =\{X+i J(X): X \in V\} .
\end{aligned}
$$

A aplicação $J: V \rightarrow V$ induz uma $J^{*}: V^{*} \rightarrow V^{*}$ por

$$
\left(J^{*} w\right)(v)=w(J v) .
$$

Claramente $\left.J^{*}=\left(J^{*}\right)^{(}-1\right)=-I$. De forma totalmente análoga a $V \otimes \mathbb{C}$, temos que

$$
V^{*} \otimes \mathbb{C}=V^{*}[i] \oplus V^{*}[-i]
$$

Usando algumas propriedades do produto tensorial (ver [1]) temos que

$$
\begin{aligned}
(V \otimes \mathbb{C}) \otimes_{\mathbb{C}}(V \otimes \mathbb{C}) \cong & \left(V[i] \otimes_{\mathbb{C}} V[i]\right) \oplus\left(V[i] \otimes_{\mathbb{C}} V[-i]\right) \oplus\left(V[-i] \otimes_{\mathbb{C}} V[i]\right) \\
& \oplus\left(V[-i] \otimes_{\mathbb{C}} V[-i]\right) \\
\left(V^{*} \otimes \mathbb{C}\right) \otimes_{\mathbb{C}}\left(V^{*} \otimes \mathbb{C}\right) \cong & \left(V^{*}[i] \otimes_{\mathbb{C}} V^{*}[i]\right) \oplus\left(V^{*}[i] \otimes_{\mathbb{C}} V^{*}[-i]\right) \oplus\left(V^{*}[-i]\right. \\
& \left.\otimes_{\mathbb{C}} V^{*}[i]\right) \oplus\left(V^{*}[-i] \otimes_{\mathbb{C}} V^{*}[-i]\right) \\
\left(V^{*} \otimes \mathbb{C}\right) \otimes_{\mathbb{C}}(V \otimes \mathbb{C}) \cong & \left(V^{*}[i] \otimes_{\mathbb{C}} V[i]\right) \oplus\left(V^{*}[-i] \otimes_{\mathbb{C}} V[-i]\right) \oplus\left(V^{*}[-i]\right. \\
& \left.\otimes_{\mathbb{C}} V[i]\right) \oplus\left(V^{*}[-i] \otimes V[-i]\right) .
\end{aligned}
$$

Usaremos bastante em nosso trabalho formas lineares a valores complexos. Definamo-las precisamente tendo em mente que $\Lambda^{k} V$ é o espaço das k-formas reais para o espaço vetorial real $V$.

Definição 1.1.1 Uma forma a valores complexos sobre um espaço vetorial real $V$ é um elemento de $\left(\Lambda^{k} V\right) \otimes \mathbb{C}$. O produto exterior em $\left(\Lambda^{k} V\right) \otimes \mathbb{C}$ é dado pela extensão linear do produto exterior de $\Lambda^{k} V$. 
Proposição 1.1.2 $\Lambda^{k} V \otimes \mathbb{C} \cong \Lambda^{k}(V \otimes \mathbb{C})$.

Demonstração: é imediata a partir das definições de $\Lambda^{k} V \otimes \mathbb{C}$ e de $\Lambda^{k}(V \otimes \mathbb{C})$.

Como conseqüência teremos que: tomando $\Lambda^{k}(V \otimes \mathbb{C})$ o espaço das $k$-formas alternadas sobre $V \otimes \mathbb{C}$ e denotando por $\Lambda(V \otimes \mathbb{C})=\sum_{r=0}^{n} \Lambda^{r}(V \otimes \mathbb{C})$, então

$$
\Lambda(V \otimes \mathbb{C})=\sum_{r=0}^{n} \sum_{p+q=r} \Lambda^{p}(V[i]) \wedge \Lambda^{q}(V[-i]) .
$$

Em um espaço vetorial $V$ de dimensão $2 n$ com uma estrutura quase-complexa $J$ podemos definir um produto interno $h$ da seguinte forma: tomemos $e_{1} \in V \backslash\{0\}$ e $e_{n+1}=J e_{1}$. É fácil verificar que $\left\{e_{1}, J e_{1}\right\}$ é L.I. Tomemos $e_{2} \in V \backslash\left[e_{1}, J e_{1}\right]$ e $e_{n+2}=J e_{2}$, analogamente ao primeiro caso $\left\{e_{1}, J e_{1}, e_{2}, J e_{2}\right\}$ é L.I., pois $\left\{e_{1}, J e_{1}, e_{2}\right\}$ é L.I. por construção. É suficiente continuar nesta direção. Construída a base $\left\{e_{1}, e_{2}, \ldots, e_{n}, J e_{1}, J e_{2}, \ldots, J e_{n}\right\}$, é suficiente impor que ela seja ortonormal ao produto interno, isto é,

$$
\begin{aligned}
h\left(e_{j}, e_{k}\right) & =\delta_{j}^{k}, \\
h\left(e_{j}, J e_{k}\right) & =0, \\
h\left(J e_{j}, J e_{k}\right) & =\delta_{k}^{j} .
\end{aligned}
$$

Se $X=\sum_{j=1}^{n} X^{j} e_{j}+X^{j+n} J e_{j}$ e $Y=\sum Y^{j} e_{j}+Y^{j+n} J e_{j}$,

$$
\begin{aligned}
h(J X, J Y) & =h\left(\sum_{j}\left(-X^{j+n} e_{j}+X^{j} J e_{j}\right), \sum_{j}\left(-Y^{j+n} e_{j}+Y^{j} J e_{j}\right)\right) \\
& =\sum_{j} X^{j+n} Y^{j+n}+X^{j} Y^{j}=h(X, Y) .
\end{aligned}
$$

Definição 1.1.3 A um produto interno $h$ sobre um espaço vetorial $V$ com uma extrutura complexa $J$ que possui $J$ como isometria denominamos Hermitiano.

Associado a $h$ temos uma forma bilinear $\omega$ definida por

$$
\omega(X, Y)=h(X, J Y) .
$$

Proposição 1.1.4 A 2-forma $\omega$ definida acima é alternada.

\section{Demonstração:}

$$
\omega(Y, X)=h(Y, J X)=-h\left(J^{2} Y, J X\right)=-h(J Y, X)=-h(X, J Y)=-\omega(X, Y) .
$$


Em coordenadas $\omega$ é dada por

$$
\begin{aligned}
\omega(X, Y) & =h\left(\sum X^{j} e_{j}+X^{j+n} J e_{j}, \sum-Y^{j+n} e_{j}+Y^{j} J e_{j}\right) \\
& =\sum_{j=1}^{n}\left(X^{j+n} Y^{j}-X^{j} Y^{j+n}\right) .
\end{aligned}
$$

Já sabemos que $J$ possui uma extensão natural para $V \otimes \mathbb{C}$. Por linearidade podemos estender $h$ a uma $\tilde{h}$ sobre $V \otimes \mathbb{C}$ e portanto faz sentido

$$
\tilde{\omega}(X, Y)=\tilde{h}(X, \tilde{J} Y) \text { para } X, Y \in V \otimes \mathbb{C} .
$$

De $V \otimes \mathbb{C}=V[i] \oplus V[-i]$ e do fato facilmente verificado que $V[i]=\{X-i J X \mid X \in V]$ e $V[-i]=\{X+i J X \mid X \in V\}$ teremos que

$$
\left\{\frac{e_{j}-i J e_{1}}{2}, \ldots, \frac{e_{h}-i J e_{n}}{2}, \frac{e_{j}+i J e_{1}}{2}, \ldots, \frac{e_{n}+i J e_{n}}{2}\right\}(*)
$$

é base de $V \otimes \mathbb{C}$. Seja $\left\{\theta^{1}, \ldots, \theta^{2 n}\right\}$ a base dual de $\left\{e_{1}, \ldots, e_{n}, J e_{1}, \ldots, J e_{n}\right\},-J^{*} \theta^{j}\left(J e_{j}\right)=$ $-\theta^{j}\left(-e_{j}\right)=1$ e $J^{*} \theta^{j}\left(J e_{k}\right)=0$ se $k \neq j$ e $J^{*} \theta^{j}\left(e_{k}\right)=0$. Portanto, $\theta^{n+j}=-J^{*} \theta^{j}$. Logo, $\left\{\theta^{1}-i J^{*} \theta^{1}, \ldots, \theta^{n}-i J^{*} \theta^{n}, \theta^{1}+i J^{*} \theta^{1}, \ldots, \theta^{n}+i J^{*} \theta^{n}\right\}$ é a base dual a $(*)$. Voltando a $\tilde{\omega}$ teremos, fazendo $f_{j}=e_{j}-i J e_{j}, \bar{f}_{j}=e_{j}+i J e_{j}, \omega^{j}=\theta^{j}+i J^{*} \theta^{j}$ e $\bar{\omega}^{j}=\theta^{j}+i J^{*} \theta^{j}$

$$
\begin{aligned}
\tilde{\omega}(X, Y) & =\tilde{h}(X, \tilde{J} Y)=\tilde{h}\left(\sum_{j} \omega^{j}(X) f_{j}+\bar{\omega}^{j}(X) \bar{f}_{j}, i\left(\sum_{j} \omega^{j}(Y) f_{j}-\bar{\omega}^{j}(Y) \bar{f}_{j}\right)\right) \\
& =2 \sum_{j} \sum_{k} i\left[\omega^{j}(X) \bar{\omega}^{k}(Y)-\omega^{j}(Y) \bar{\omega}^{k}(X)\right] \tilde{h}\left(f_{j}, \bar{f}_{k}\right) .
\end{aligned}
$$

Portanto, denotando $h_{j \bar{k}}=h\left(f_{j}, \bar{f}_{k}\right)$ teremos $\tilde{\omega}=2 i \sum_{j \leq k} h_{j \bar{k}} \omega^{j} \wedge \bar{\omega}^{k}$.

Proposição 1.1.5 A forma $\omega$ definida acima é tal que $\omega^{n}=\omega \wedge \ldots \wedge \omega$ é um múltiplo da forma volume do $\mathbb{R}^{2 n}$.

Demonstração: a proposição é imediata a partir do fato que: em relação à base $\left\{\theta^{1}, \ldots, \theta^{2 n}\right\}$, $\omega$ se escreve da seguinte forma

$$
\omega=\theta^{1} \wedge \theta^{n+1}+\theta^{2} \wedge \theta^{n+2}+\cdots+\theta^{n} \wedge \theta^{2 n}
$$


Da forma como foi definido o $\mathbb{C}^{n}$ possui uma topologia natural: a topologia do $\mathbb{R}^{2 n}$.

Definição 1.1.6 Uma função $f: U \rightarrow \mathbb{C}$ definida sobre o aberto $U \subset \mathbb{C}^{n}$ é denominada holomorfa se como uma aplicação de $U \subset \mathbb{R}^{2 n}$ em $\mathbb{R}^{2}$ ela é $\mathcal{C}^{\infty}$ e satifaz as equações de Cauchy-Riemann

$$
\begin{aligned}
& \frac{\partial(\operatorname{Re}(f))}{\partial x_{j}}=\frac{\partial(\operatorname{Im}(f))}{\partial y_{j}} \\
& \frac{\partial(\operatorname{Re}(f))}{\partial y_{j}}=-\frac{\partial(\operatorname{Im}(f))}{\partial x_{j}}
\end{aligned}
$$

para $j=1, \ldots, n$. Uma aplicação de $f: U \rightarrow \mathbb{C}^{n}$ será denominada holomorfa se cada uma de suas componentes é holomorfa.

Definição 1.1.7 Dada uma aplicação de $f$ de um aberto de $\mathbb{C}^{n}$ em $\mathbb{C}^{m}$ dizemos que ela é compatível com as estruturas complexas de $\mathbb{C}^{n}$ e $\mathbb{C}^{m}$ se

$$
d f \circ J=J \circ d f
$$

sendo df a diferencial da aplicação $f$.

Lema 1.1.8 Uma aplicação $f: U \subset \mathbb{C}^{n} \rightarrow \mathbb{C}^{m}, \mathcal{C}^{\infty}$ como uma aplicação do $\mathbb{R}^{2 n}$ em $\mathbb{R}^{2 m}$, será holomorfa se e somente se ela for compatível com as estruturas complexas de $\mathbb{C}^{n} e \mathbb{C}^{m}$.

Demonstração: Imediata

Definição 1.1.9 Uma estrutura complexa sobre uma variedade $M$ de classe $\mathcal{C}^{\infty}$ e dimensão par é uma família maximal de cartas $\left\{\left(\varphi_{\lambda}, U_{\lambda}\right): \varphi_{\lambda}: U_{\lambda} \rightarrow \mathbb{R}^{2 n}\right\}$ tal que $\bigcup U_{\lambda}=M$ e satisfazem a condição de transição holomorfa, isto é, para todo par de indices $\lambda$ e $\mu$ teremos

$$
\varphi_{\lambda} \circ \varphi_{\mu}^{-1}: \varphi_{\mu}\left(U_{\lambda} \cap U_{\mu}\right) \rightarrow \varphi_{\lambda}\left(U_{\lambda} \cap U_{\mu}\right)
$$

é uma aplicação holomorfa quando considerada como uma aplicação de $\mathbb{C}^{n}$ em $\mathbb{C}^{n}$.

Definição 1.1.10 Uma variedade complexa $M$ é uma variedade diferenciável $M$ sobre a qual é dada uma estrutura complexa. As cartas que compõem a estrutura complexa denominamos de cartas holomorfas. 
Definição 1.1.11 Uma estrutura quase-complexa em uma variedade diferenciável $M$ consiste em um campo tensorial $J, \mathcal{C}^{\infty}$ de tipo $(1,1)$, tal que $J^{2}=-I$.

Lema 1.1.12 Qualquer conjunto de cartas cujo domínio cobre a variedade $M$ e satisfaz a propriedade de transição holomorfa determina uma única estrutura quase-complexa sobre $M$.

Demonstração: para uma variedade complexa $M$ seja $T_{p} M$ o seu espaço tangente (do ponto de vista real) no ponto $p$ e $\varphi: U \rightarrow \mathbb{R}^{2 n}$ uma carta holomorfa tal que $p \in U$. Podemos então definir um operador linear $J_{p}$ de $T_{p} M$ em $T_{p} M$ da seguinte maneira

$$
J_{p}=d \varphi_{\varphi(p)}^{-1} \circ J_{\mathbb{R}} \circ d \varphi_{p}
$$

É imediato que $J_{p}$ independe da carta pela qual ele foi definido. Portanto, temos um campo tensorial $J_{M}$ de tipo $(1,1)$ definido sobre $M$ e ele é tal que $J_{M}^{2}=-I$.

Definição 1.1.13 Sejam $M$ e $N$ duas variedades complexas e $f: M \rightarrow N$ uma aplicação de classe $\mathcal{C}^{\infty}$. Dizemos que $f$ é holomorfa se $d f \circ J_{M}=J_{N} \circ d f$.

Não demonstraremos neste trabalho, mas é verdadeiro que se uma função $f: M \rightarrow N$, entre duas variedades complexas $M$ e $N$, é holomorfa no sentido da definição acima, então para todos os pares de cartas holomorfas $\varphi$ de $M$ e $\psi$ de $N$ para os quais faz sentido

$$
\psi \circ f \circ \varphi^{-1}
$$

esta será holomorfa no sentido da definição 1.1.6.

Sendo $\Lambda^{k} M$ o conjunto das k-formas diferenciais da variedade $M$ temos:

Definição 1.1.14 Uma $k$-forma a valores complexos sobre uma variedade diferenciável $M$ é um elemento de $\left(\Lambda^{k} M\right) \otimes \mathbb{C}$. O produto exterior em $\left(\Lambda^{k} M\right) \otimes \mathbb{C}$ é dado pela extensão linear do produto exterior de $\Lambda^{k} M$. 


\section{Exemplos:}

$\mathbb{C}^{n}$ : Identificando $p \in \mathbb{R}^{2 n}$ com suas coordenadas canônicas, isto é, $p=\left(x_{1}, \ldots, x_{n}, y_{1}, \ldots, y_{n}\right)$ podemos definir $\varphi: \mathbb{C}^{n} \rightarrow \mathbb{R}^{2 n} \operatorname{por} \varphi(v)=v$. Também podemos representar $p \in \mathbb{C}^{n}$ da seguinte forma:

$$
p=\sum_{j=1}^{n} x_{j} e_{j}+\sum_{j=1}^{n} y_{j} e_{n+j}=\sum_{j=1}^{n}\left(x_{j} e_{j}+y_{j} J e_{j}\right)=\sum_{j=1}^{n}\left(x_{j}+i y_{j}\right) e_{j}=\left(z_{1}, \ldots, z_{n}\right),
$$

sendo $z_{j}=x_{j}+i y_{j}$ e logo teremos, se olharmos $\varphi$ como uma aplicação de $\mathbb{C}^{n}$ em $\mathbb{C}^{n}$, $\varphi(p)=\left(z_{1}, \ldots, z_{n}\right)$ se $p=\left(z_{1}, \ldots, z_{n}\right)$ como $\varphi \varphi^{-1}$ é a identidade, segue que $\mathbb{C}^{n}$ é uma variedade complexa.

Superfícies orientáveis: Toda superfície orientável pode ser dotada de uma estrutura complexa unidimensional. De fato, podemos assumir a existência de uma métrica Riemanniana $g$ e de coordenadas tais que, localmente

$$
g=\lambda(d x \otimes d x+d y \otimes d y)
$$

para uma certa função $\lambda$ positiva. É suficiente definir $\phi(p)=x+i y$ para $p$ no domínio das coordenas $(x, y)$. Se $\psi$ é outra carta e $z=x+i y$, teremos

$$
\lambda d z \wedge d \bar{z}=\mu d w \wedge d \bar{w}
$$

supondo que $\phi$ e $\psi$ determinam a mesma orientação obtém-se $d w=f(z) d z$, para uma certa função holomorfa $f$.

Toros complexos: Sejam $v_{1}, \ldots, v_{2 n}$ vetores de $\mathbb{C}^{n}$ linearmente independentes sobre $\mathbb{R}$ e $\Gamma$ um grupo disctreto de transformações da forma

$$
z \longrightarrow z+\sum_{\alpha=1}^{2 n} N_{\alpha} v_{\alpha}
$$

sendo, $N_{\alpha}$ tomado em $\mathbb{Z}$. Definamos em $\mathbb{C}^{n}$ a seguinte relação de equivalência em $\mathbb{C}^{n}$, $z \sim z^{\prime}$ se existe uma transformação $f$ em $\Gamma$ tal que $z^{\prime}=f(z)$. O conjunto $\mathbb{C}^{n} / \Gamma$ das classes de equivalências possui uma estrutura de variedade complexa. Em verdade, verifica-se que existe para todo ponto $z \in \mathbb{C}^{n}$ uma vizinhança $\widetilde{U}$ satisfazendo $h(\widetilde{U}) \cap \widetilde{U}=\varnothing$ para todo $h \in(\Gamma-\{e\})$. Portanto, a projeção canônica $\pi$ restrita a $\widetilde{U}$ é injetora. Supondo $\widetilde{U}$ conexo é suficiente definir as cartas $(U, \psi)$ em $\mathbb{C}^{n} / \Gamma$ por $U=\pi(\widetilde{U})$ e $\psi=(\pi \mid \widetilde{U})^{-1}$. 


\subsection{O espaço projetivo complexo}

Seja $\mathbb{C}^{n+1}$ o espaço complexo $n+1$-dimensional. Definamos $S_{r}^{2 n+1}=\left\{z \in \mathbb{C}^{2 n+1} \mid\langle z, z\rangle=\right.$ $\left.z_{0} \bar{z}_{0}+\cdots+z_{n} \bar{z}_{n}=r^{2}\right\}$. É fato assumido neste trabalho que $S^{2 n+1}$ possui uma estrutura de variedade diferenciável para todo $n \in \mathbb{N}$. Podemos também verificar que a aplicação que associa a cada $(z, \lambda) \in S^{2 n+1} \times S^{1}$ o elemento $R(z, \lambda)=\lambda z \in S^{2 n+1}$ é uma ação à direita do grupo de Lie $S^{1}$ sobre $S^{2 n+1}$. De fato, $R(z, 1)=z, R(R(w, \zeta) \lambda)=R(\zeta w, \lambda)=$ $\lambda \zeta w=\zeta \lambda w=R(w, \zeta \lambda)$. Que a ação $R$ pertence à $\mathcal{C}^{\infty}\left(S_{r}^{2 n+1} \times S^{1}, S_{r}^{2 n+1}\right)$ é óbvio. É imediato também que fixado $\lambda \in S^{1}, R_{\lambda}: S^{2 n+1} \rightarrow S^{2 n+1}$ é um difeomorfismo. Sob esta ação $R, S^{1}$ age livremente sobre $S^{2 n+1}$, isto é, se $R(z, \lambda)=z$ para algum $z \in S_{r}^{2 n+1}$, então $\lambda=1$. Em outras palavras, se definirmos $[z]=\left\{w \in S_{r}^{2 n+1} \mid w=R(z, \lambda)\right.$ para algum $\left.\lambda \in S^{1}\right\}$ teremos para $z$ e $z^{\prime}$ em $S_{r}^{2 n+1}$ diferentes $[z]=\left[z^{\prime}\right]$ ou $[z] \cap\left[z^{\prime}\right]=\emptyset$. De fato, se existe $\lambda$ tal que $z^{\prime}=r(z, \lambda)$ e $w \in\left[z^{\prime}\right]$, então por definição $w=R\left(z^{\prime}, \zeta\right)$ para algum $\zeta$, portanto $w=R\left(z^{\prime}, \zeta\right)=R(R(z, \lambda), \zeta)=R(z, \lambda \zeta) \in[z]$, isto é, $\left[z^{\prime}\right] \subset[z]$. De $z=R\left(z^{\prime}, \frac{1}{\lambda}\right)$ segue que $[z] \subset\left[z^{\prime}\right]$, isto é, $[z]=\left[z^{\prime}\right]$. Se não existe $\lambda \in S^{1}$ tal que $z^{\prime}=R(z, \lambda)$ e $[z] \cap\left[z^{\prime}\right] \neq \emptyset$, teremos, tomando $w$ em $[z] \cap\left[z^{\prime}\right]$, que existem $\left(\lambda_{1}, \lambda_{2}\right) \in S^{1} \times S^{1}$, tal que $R\left(z, \lambda_{1}\right)=w=R\left(z^{\prime}, \lambda_{2}\right)$, portanto $\left.R\left(w, \frac{1}{\lambda_{1}}\right)=R\left(R\left(z, \lambda_{1}\right), \frac{1}{\lambda_{1}}\right)=R(z, 1)=z\right)$. Por outro lado, $R\left(w, \frac{1}{\lambda_{1}}\right)=R\left(R\left(z^{\prime}, \lambda_{2}\right), \frac{1}{\lambda_{1}}\right)=R\left(z^{\prime}, \frac{\lambda_{2}}{\lambda_{1}}\right)$, o que é um absurdo. Podemos então definir o seguinte conjunto $\mathbb{C P}^{n}\left(\frac{4}{r^{2}}\right)=\left\{[z] \mid z \in S^{2 n+1}\right\}$ e $\pi: S_{r}^{2 n+1} \rightarrow \mathbb{C P}^{n}\left(\frac{4}{r^{2}}\right)$ por $\pi(z)=[z]$. Dotamos $\mathbb{C P}^{n}\left(\frac{4}{r^{2}}\right)$ da seguinte topologia: um subconjunto $U \subset \mathbb{C P}^{n}\left(\frac{4}{r^{2}}\right)$ é aberto se $\pi^{-1}(U)$ é aberto em $S_{r}^{2 n+1}$. É fácil verificar que isto realmente dota $\mathbb{C P}^{n}\left(\frac{4}{r^{2}}\right)$ de uma topologia. Podemos ir ainda mais longe e dotar $\mathbb{C P}^{n}\left(\frac{4}{r^{2}}\right)$ de uma estrutura complexa.

Comecemos a construção da estrutura definindo $U_{i}=\left\{[z] \in \mathbb{C P}^{n}\left(\frac{4}{r^{2}}\right) \mid z_{i} \neq 0\right\}$, $i=1,2, \ldots, n$. $U_{i}$ é aberto. Com efeito, $\pi^{-1}\left(U_{j}\right)=\left\{z \in S_{r}^{2 n+1} \mid z_{j} \neq 0\right\}$. Definamos $\varphi_{j}: U_{j} \rightarrow \mathbb{R}^{2 n}$ por

$$
\varphi_{j}([z])=\frac{1}{z_{j}}\left(z_{1}, \ldots, \hat{z}_{j}, \ldots, z_{n+1}\right) .
$$

$\varphi_{j}$ está bem definida, $\varphi$ é claramente injetora, pois e $[w]=[z]$, teremos $w=\lambda z$ e portanto

$$
\begin{aligned}
\varphi_{j}([w]) & =\frac{1}{w_{j}}\left(w_{1}, \ldots, \hat{w}_{j}, \ldots, w_{n+1}\right)=\frac{1}{\lambda z_{j}}\left(\lambda z_{1}, \ldots, \lambda \hat{z}_{j}, \ldots, \lambda z_{n+1}\right) \\
& =\frac{1}{z_{1}}\left(z_{1}, \ldots, \hat{z}_{j}, z_{n+1}\right)=\varphi_{j}([z]) .
\end{aligned}
$$

$\operatorname{Em} U_{k} \cap U_{j}=\left\{[z] \in \mathbb{C P}^{n}\left(\frac{4}{r^{2}}\right)\left(\mid z_{k} \neq 0\right.\right.$ e $\left.z_{j} \neq 0\right\}$ fará sentido $\varphi_{k}\left(U_{k} \cap U_{j}\right)$ e este conjunto é igual a $\mathbb{C}^{n} \backslash\left\{\left(z_{1}, \ldots, \hat{z}_{k}, \ldots, z_{n}\right) \in \mathbb{C}^{N} \mid z_{j}=0\right\}$, que é aberto. Faz sentido então $\varphi_{j} \circ \varphi_{k}^{-1}: \mathbb{C}^{n} \backslash\left\{z \in \mathbb{C}^{n} \mid z_{j}=0\right\} \rightarrow \mathbb{C}^{n}$ e $\varphi_{j} \circ \varphi_{k}^{-1}\left(z_{1}, \ldots, z_{n}\right)=\frac{1}{z_{j}}\left(z_{1}, \ldots, 1, \ldots, z_{n}\right)$ é claramente $\mathcal{C}^{\infty}$. Na verdade, $\varphi_{j} \circ \varphi_{k}^{-1}$ é mais do que diferenciável, ela é holomorfa. Como 
$\bigcup_{j=1}^{n} U_{j}=\mathbb{C P P}^{n}\left(\frac{4}{r^{2}}\right)$ e $\varphi_{j} \circ \varphi_{k}^{-1}$ é diferenciável se $i \neq j$, tomando a família maximal de cartas que contêm $\left\{\varphi_{1}, \ldots, \varphi_{n+1}\right\}$ teremos uma estrutura complexa sobre $\mathbb{C P}^{n}\left(\frac{4}{r^{2}}\right)$.

$\left(S_{r}^{2 n+1}, S^{1}, \pi, \mathbb{C P}^{n}\left(\frac{4}{r^{2}}\right)\right)$ esta quadra possui algumas propriedades que explicitaremos. Já sabemos que a ação à direita $R$ do $S^{1}$ sobre $S_{r}^{2 n+1}$ age livremente e diferenciavelmente. $\pi$ é uma aplicação $\mathcal{C}^{\infty}$ de $S_{r}^{2 n+1}$ sobre $\mathbb{C P}^{n}\left(\frac{4}{r^{2}}\right)$. De fato, esta afirmação decorre do fato que a aplicação de $S^{2 n+1} /\left\{z \mid z_{i}=0\right\}$ em $\mathbb{C}^{n}$, que associa a cada $z, \frac{1}{z_{i}}\left(z_{1}, \ldots, \hat{z}_{i}, \ldots, z_{n+1}\right)$ é diferenciável e igual a $\varphi_{i} \circ \pi$. Também é evidente que $S^{1}$ age transitivamente sobre $\pi^{-1}([z])$ para todo $[z] \in \mathbb{C P}^{n}\left(\frac{4}{r^{2}}\right)$. Menos evidente que as propriedades acima é a trivialidade local para a quadra $\left(S_{r}^{2 n+1}, S^{1}, \pi, \mathbb{C P}^{n}\left(\frac{4}{r^{2}}\right)\right)$, isto é, para todo $[z] \in \mathbb{C I P}^{n}\left(\frac{4}{r^{2}}\right)$ podemos tomar um aberto $U_{i} \subset \mathbb{C P}^{n}\left(\frac{4}{r^{2}}\right)$ tal que $[z] \in U_{i}$ e $\pi^{-1}\left(U_{i}\right)$ e $U_{1} \times S^{1}$ são difeomorfas. Em verdade, podemos definir uma $f: \pi\left(U_{j}\right) \rightarrow S^{1}$ dada por $f(z)=(\pi(z), F(z))$, sendo $F_{j} \pi^{-1}\left(U_{j}\right) \rightarrow S^{1}$ uma aplicação $\mathcal{C}^{\infty}$ tal que $F_{j}(R(w, \lambda))=F_{j}(w) \lambda$, dada por $F_{j}(z)=z_{j} /\left|z_{j}\right|$. A quadra $\left(S_{r}^{2 n+1}, S^{1}, \pi, \mathbb{C P}^{n}\left(\frac{4}{r^{2}}\right)\right)$ com as propriedades anteriores é o que se denomina de um fibrado principal.

Para $z \in S_{r}^{2 n+1}$ determinaremos $T_{z} S^{2 n+1}$. Se $\gamma(-\varepsilon, \varepsilon) \rightarrow S^{2 n+1}$ é uma curva tal que $\gamma(0)=z$ e $\gamma(0)=v$ teremos, diferenciando $\langle\gamma(t), \gamma(t)\rangle=r^{2},\left\langle\gamma(t), \gamma^{\prime}(t)\right\rangle+\left\langle\gamma^{\prime}(t), \gamma(t)\right\rangle=0$, para $t=0$ obteremos $\langle z, v\rangle+\langle v, z\rangle=0(\operatorname{Re}\langle z, v\rangle=0)$. Reciprocamente, se $v \in \mathbb{C}^{n+1}$ e $R e\langle z, v\rangle=0$ para $z \in S_{r}^{2 n+1}$, então $v \in T_{z} S_{r}^{2 n+1}$. Em verdade, façamos $\gamma:(-\varepsilon, \varepsilon) \rightarrow$ $S_{r}^{2 n+1}$ tal que $\gamma(t)=r \frac{z+t v}{\|z+t v\|}$. Um cálculo simples nos mostra que $\gamma^{\prime}(0)=v$ e portanto $T_{z} S_{r}^{2 n+1}=\left\{v \in \mathbb{C}^{n+1} \mid \operatorname{Re}\langle z, v\rangle=0\right\}$. Podemos em $S^{2 n+1}$ definir uma distribuição $\mathcal{D}$ como sendo $\mathcal{D}(z)=\left\{v \in T_{z} S_{r}^{2 n+1} \mid\langle z, v\rangle=0\right\}$, teremos então que $\operatorname{dim} \mathcal{D}(z)=2 n$ sobre $\mathbb{R}$. Um fato que será muito usado é que se $v \in \mathcal{D}(z)$, então $i v \in \mathcal{D}(z)$.

Fazendo $\xi_{z}=\frac{i z}{r}$, teremos $\left\langle z, \xi_{z}\right\rangle=\frac{i}{r}\langle z, z\rangle=i\|z\|^{2}$ e portanto $\xi_{z} \in T_{z} S^{2 n+1}$ e $\xi_{z} \notin \mathcal{D}(z)$. Para a aplicação $\pi$ temos associado $d \pi_{z}: T_{z} S_{r}^{2 n+1} \rightarrow T_{[z]} \mathbb{C P}^{n}\left(\frac{4}{r^{2}}\right) . d \pi_{z}\left(\xi_{z}\right)=0$, pois se $\gamma(t)=e^{i t} z, \gamma^{\prime}(0)=r \xi_{z}$. Por definição teremos

$$
d \varphi_{j} \pi_{z}\left(\xi_{z}\right)=\left.\frac{d}{d t}\left(\varphi_{j} \pi \circ \gamma(t)\right)\right|_{t=0}=\left.\frac{d}{d t} \varphi_{j} \pi\left(e^{i t} z\right)\right|_{t=0}=\left.\frac{d}{d t} \varphi_{j}([z])\right|_{t=0}=0 .
$$

Podemos então concluir que $\left.d \pi_{z}\right|_{\mathcal{D}(z)}: \mathcal{D}(z) \rightarrow T_{[z]} \mathbb{C P}^{n}\left(\frac{4}{r^{2}}\right)$ é um isomorfismos e isto nos possibilitará munir $\mathbb{C P}^{n}\left(\frac{4}{r^{2}}\right)$ com um produto sesquilinear do qual obteremos uma métrica sobre $\mathbb{C P}^{n}\left(\frac{4}{r^{2}}\right)$. Para $[z] \in \mathbb{C P}^{n}\left(\frac{4}{r^{2}}\right)$ a aplicação $[z] \rightarrow z \in \mathbb{C}^{n+1}$ denomina-se coordenada homogênea de $[z]$, enquanto $[z] \in U_{i} \rightarrow \frac{1}{z_{i}}\left(z_{1}, \ldots, \hat{z}_{i}, \ldots, z_{n+1}\right) \in \mathbb{C}^{n}$ denomina-se coordenada não-homogênea de $[z] \in U_{i}$. Tomemos uma curva $\gamma \in \mathcal{C}^{\infty}, \gamma:(-\varepsilon, \varepsilon) \rightarrow S_{r}^{2 n+1}$, $\operatorname{com} \gamma(0)=z$ e $\gamma^{\prime}(0)=v . \quad \tilde{\gamma}(t)=\pi \circ \gamma(t)$ é uma curva $\mathcal{C}^{\infty}$ em $\mathbb{C P}^{n}\left(\frac{4}{r^{2}}\right)$. Para vetores $u, v \in T_{[z]} \mathbb{C P}^{n}\left(\frac{4}{r^{2}}\right)$ sabemos que existem $\tilde{u}, \tilde{v} \in T_{z} S^{2 n+1}$ tais que $u=d \pi_{z}(\tilde{u})$ e $v=d \pi_{z}(\tilde{v})$. Portanto, podemos definir a aplicação bilinear $\langle\langle\rangle$,$\rangle por \langle\langle u, v\rangle\rangle_{[z]}=\langle\tilde{u}, \tilde{v}\rangle_{z}$. Este produto 
está bem definido. Com efeito, se $w \in S_{r}^{2 n+1}$ e $\pi(w)=[z]$, então existem $\tilde{u}_{1}$ e $\tilde{v}_{1} \in T_{w} S_{r}^{2 n+1}$ tais que $u=d \pi_{w}\left(\tilde{u}_{1}\right)$ e $v=d \pi_{w}\left(\tilde{v}_{1}\right)$. Portanto, basta mostrar que $\langle\tilde{u}, \tilde{v}\rangle_{z}=\left\langle\tilde{u}_{1}, \tilde{v}_{1}\right\rangle_{w}$, o que é elementar, pois $w=\lambda z$ para algum $\lambda \in S^{1}$ e como $R_{\lambda}$ é tal que $d R_{\lambda}(v)=\lambda v$, segue o que queremos, isto é, $\langle\tilde{u}, \tilde{v}\rangle_{z}=\left\langle\tilde{u}_{1}, \tilde{v}_{1}\right\rangle$. Escreveremos agora $\left\langle\langle v, v\rangle_{[z]}\right.$ em coordenadas. Para tal, seja $\tilde{v} \in T_{z} S_{r}^{2 n+1}$ tal que $d \pi_{z}(\tilde{v})=v$ e seja $\gamma(-\varepsilon, \varepsilon) \rightarrow S_{r}^{2 n+1}$ tal que $\gamma(0)=z \mathrm{e}$ $\gamma^{\prime}(0)=\tilde{v}$. A componente de $\gamma^{\prime}(0)$ em $\mathcal{D}(z)$ é dada por $\left.\left.\gamma^{\prime}(0)-\right\rangle \gamma^{\prime}(0), \xi_{z}\right) \xi_{z}$ e portanto

$$
\begin{aligned}
\langle\langle v, v\rangle\rangle & =\left\langle\gamma^{\prime}(0)-\left\langle\gamma^{\prime}(0), \xi_{z}\right\rangle \xi_{z}, \gamma^{\prime}(0)-\left\langle\gamma^{\prime}(0), \xi_{z}\right\rangle \xi_{z}\right\rangle \\
& =\left\langle\gamma^{\prime}(0), \gamma^{\prime}(0)\right\rangle-\left|\left\langle\gamma^{\prime}(0), \xi_{z}\right\rangle\right|^{2} \\
& =\left\langle\gamma^{\prime}(0), \gamma^{\prime}(0)\right\rangle-\frac{1}{r^{2}}\left|\left\langle\gamma^{\prime}(0), z\right\rangle\right|^{2} .
\end{aligned}
$$

Conseqüentemente, se tomarmos uma curva $\tilde{\gamma}:(-\varepsilon, \varepsilon) \rightarrow \mathbb{C}^{n+1}$ tal que $\gamma(t)=r \frac{\widetilde{\gamma(t)}}{\|\widetilde{\gamma}(t)\|}$,

$$
\gamma^{\prime}(t)=r \frac{2\|\tilde{\gamma}(t)\|^{2} \tilde{\gamma}^{\prime}(t)-\left(\left\langle\tilde{\gamma}(t), \tilde{\gamma}^{\prime}(t)\right\rangle+\left\langle\tilde{\gamma}^{\prime}(t), \tilde{\gamma}(t)\right\rangle\right) \tilde{\gamma}(t)}{2\|\tilde{\gamma}\|^{3}}
$$

e portanto para $\langle\langle$,$\rangle obteremos$

$$
\left\langle\left\langle d \pi_{\gamma(t)}\left(\gamma^{\prime}(t)\right), d \pi_{\gamma(t)}\left(\gamma^{\prime}(t)\right)\right\rangle\right\rangle=r^{2} \frac{\|\tilde{\gamma}(t)\|^{2}\left\|\tilde{\gamma}^{\prime}(t)\right\|^{2}-\left|\left\langle\tilde{\gamma}(t), \tilde{\gamma}^{\prime}(t)\right\rangle\right|^{2}}{\|\tilde{\gamma}(t)\|^{4}} .
$$

Formalmente, podemos escrever

$$
\pi^{*}\left\langle\langle,\rangle=r^{2} \frac{\langle z \wedge d z, z \wedge d z\rangle}{\|z\|^{4}}\right.
$$

sendo $\langle z \wedge d z, z \wedge d z\rangle=\langle z, z\rangle\langle d z, d z\rangle-\langle z, d z\rangle\langle d z, z\rangle$.

Como já sabemos, $\left.d \pi_{z}\right|_{\mathcal{D}_{z}}: \mathcal{D}_{z} \rightarrow T_{[z]} \mathbb{C P}^{n}\left(\frac{4}{r^{2}}\right)$ é um isomorfismo. Também sabemos que se $v \in \mathcal{D}_{z}$ o mesmo é verdade para $i v$, isso nos leva a definir um campo tensorial $J$ do tipo $(1,1)$ sobre $\mathbb{C P}^{n}\left(\frac{4}{r^{2}}\right)$ da seguinte maneira: $J_{[z]}\left(d \pi_{z}(v)\right)=d \pi_{z}(i v) . J$ definido desta forma é um tensor $\mathcal{C}^{\infty}$ e $J^{2}(d \pi(v))=d \pi(v)$ e, portanto, uma estrutura quase-complexa de $\mathbb{C P}^{n}\left(\frac{4}{r^{2}}\right)$. A estrutura quase-complexa induzida pelas cartas holomorfas do $\mathbb{C P}^{n}\left(\frac{4}{r^{2}}\right)$ e a induzida por $d \pi$ são equivalentes. É um fato, não difícil de demonstrar, que toda variedade que possui um tensor $J$ de tipo $(1,1)$ que é $\mathcal{C}^{\infty}$ e $J^{2}=-I$ é orientável e portanto $\mathbb{C P}^{n}\left(\frac{4}{r^{2}}\right)$ é orientável. Uma orientação é dada pela seguinte forma volume. Defina $\omega \in \Lambda^{2}\left(\mathbb{C P}^{n}\left(\frac{4}{r^{2}}\right)\right)$ por

$$
\omega(X, Y)=\langle\langle X, J Y\rangle\rangle
$$

e façamos $\eta=\omega \wedge \cdots \wedge \omega(n$-vezes $)$. 
Sejam $\left\{e_{0} ; \ldots ; e_{n}\right\}$ a base canônica de $\mathbb{C}^{n+1}$ e $U(n+1)$ o grupo unitário. Podemos então definir $n+1$ funções de $U(n+1)$ em $S^{2 n+1}$,

$$
\begin{gathered}
U(n+1) \stackrel{b_{j}}{\longrightarrow} S^{2 n+1} \subset \mathbb{C}^{n+1} \\
g \longrightarrow g\left(e_{j}\right)=\sum_{k=0}^{n} g_{j}^{k} e_{k}
\end{gathered}
$$

A $\left\{b_{0} ; \ldots ; b_{n}\right\}$ denominaremos um referencial unitário. Podemos então diferenciar $b_{j}$, do ponto de vista complexo, e obteremos

$$
d b_{j}=\sum_{k=0}^{n} \omega_{j}^{k} \otimes b_{k}, \quad j=0, \ldots, n,
$$

sendo $\omega_{j}^{k} 1$-formas complexas, isto é, $\left(\omega_{j}^{k}\right)_{g}$ é uma forma $\mathbb{C}$-linear. Verifica-se que $\omega_{j}^{k}+\overline{\omega_{k}^{j}}=$ 0 . Em geral, para referenciais unitários temos que $\left\langle b_{j}(g), b_{k}(g)\right\rangle=\delta_{j}^{k}$, diferenciando a última igualdade e aplicando em $v \in T_{g} U(n+1)$, temos que

$$
\left\langle\left(d b_{j}\right)_{g}(v), b_{k}(g)\right\rangle+\left\langle b_{j}(g),\left(d b_{k}\right)_{g}(v)\right\rangle=0 .
$$

Portanto, $\left(\omega_{j}^{k}\right)_{g}(v)+\overline{\left(\omega_{k}^{j}\right)_{g}(v)}=0$. Diferenciando exteriomente $d b_{j}=\Sigma \omega_{j}^{k} \otimes b_{k}$, teremos levando em consideração que $b_{j}=b_{j}^{l} e_{l}$

$$
\begin{aligned}
& d b_{j}=\sum_{k} \omega_{j}^{k} \otimes b_{k}=\sum_{k, l} \omega_{j}^{k} b_{k}^{l} \otimes e_{l}, \\
& d b_{j}^{l}=\sum_{k} \omega_{j}^{k} b_{k}^{l}, \\
& d\left(d b_{j}^{l}\right)=\sum_{k} d \omega_{j}^{k} b_{k}^{l}-\omega_{j}^{k} \wedge d b_{k}^{l}, \\
& \sum_{k}\left[b_{k}^{l} d \omega_{j}^{k}-\sum_{m} b_{m}^{l} \omega_{j}^{k} \wedge \omega_{k}^{m}\right]=0, \\
& \sum_{l}\left\{\sum_{k} b_{k}^{l} d \omega_{j}^{k}-\sum_{k, m} b_{m}^{l} \omega_{j}^{k} \wedge \omega_{k}^{m}\right\} \otimes e_{l}=0, \\
& \sum_{k} d \omega_{j}^{k} \otimes b_{k}-\sum_{k, m} \omega_{j}^{k} \wedge \omega_{k}^{m} \otimes b_{m}=0, \\
& \sum_{k}\left(d \omega_{j}^{k}-\sum_{m} \omega_{j}^{m} \wedge \omega_{m}^{k}\right) \otimes b_{k}=0, \\
& d \omega_{j}^{k}-\sum_{m} \omega_{j}^{m} \wedge \omega_{m}^{k}=0 .
\end{aligned}
$$

Seja agora $\pi: U(n+1) \rightarrow S_{r}^{2 n+1}$ dado por $\pi(g)=g\left(r e_{0}\right)=r b_{0}$. Mostraremos que $\left(U(n+1), U(n), S^{2 n+1}, \pi\right)$ é um $U(n)$-fibrado principal. Para tal fim, necessitamos verificar os seguintes fatos: 
$1^{\underline{o}-} U(n)$ age à direita em $U(n+1)$.

$$
\begin{aligned}
& R: U(n+1) \times U(n) \rightarrow U(n+1) \\
& R(g, h)=g \cdot\left(\begin{array}{ll}
1 & 0 \\
0 & h
\end{array}\right) .
\end{aligned}
$$

Esta ação é livre, isto é, se $g \cdot\left(\begin{array}{ll}1 & 0 \\ 0 & h\end{array}\right)=g$, então $h=I_{k}$ em $U(n)$. Também temos que a ação acima é $\mathcal{C}^{\infty}$. Que a ação é transitiva nas fibras decorre do fato que: dados dois pontos $f$ e $g$ na mesma fibra, isto é, na órbita de um $g_{0} \in U(n+1)$, então

$$
f=g_{0}\left(\begin{array}{ll}
1 & 0 \\
0 & h
\end{array}\right) \quad g=g_{0}\left(\begin{array}{ll}
1 & 0 \\
0 & k
\end{array}\right)
$$

portanto, temos $g=f\left(\begin{array}{cc}1 & 0 \\ 0 & h^{-1} k\end{array}\right)$

$2^{\underline{0}}$ - A ação é localmente trivial. Seja $w=\left(w_{0}, \ldots, w_{n}\right) \in S_{r}^{2 n+1}$. Como $w \neq 0$ existe um $w_{j} \neq 0$, suponhamos que seja $w_{0}$, os outros casos são análogos. Consideremos então $U_{0}=\left\{z \in S_{r}^{2 n+1}: z_{0} \neq 0\right\}$ é um aberto e contém $w$. Fazendo $\left(w_{0}, \ldots, w_{n}\right)=\left(w_{0}, z\right)$ teremos

$$
\begin{aligned}
& U_{0} \times U(n) \stackrel{f}{\longrightarrow} \pi^{-1}\left(U_{0}\right) \\
& \left(\left(w_{0}, z\right), h\right) \stackrel{f}{\longrightarrow}\left(\begin{array}{cc}
w_{0} & z \\
\frac{1}{w_{0}} h \bar{z}^{t} & h
\end{array}\right)
\end{aligned}
$$

Não é difícil mostrar que $f$ é um difeomorfismo.

Tomemos $g: S_{r}^{2 n+1} \rightarrow U(n+1)$ tal que $\pi \circ g(x)=x$ e $g \in \mathcal{C}^{\infty}\left(S_{r}^{2 n+1}, U(n+1)\right)$, isto é, $g$ é uma seç̧ão do fibrado principal acima. A partir do que fizemos, temos que

$$
\left(x, b_{1}[g(x)], \ldots, b_{n}[g(x)]\right)
$$

é um referencial unitário tangente a $S^{2 n+1}$. Temos também que

$$
T_{r e_{0}} S^{2 n+1}=\left[\left\{i e_{0}, e_{1}, e_{2}, \ldots, e_{n}\right\}\right] .
$$

De $\pi \circ g(x)=x$ tiramos que

$$
g(x): T_{r e_{0}} S^{2 n+1} \rightarrow T_{x} S^{2 n+1}
$$


e portanto

$$
\left\{g(x)\left(i e_{0}\right), g(x)\left(e_{1}\right), \ldots, g(x)\left(e_{n}\right)\right\}
$$

é uma base de $T_{x} S^{2 n+1}$. De $x=r b_{0}[g(x)]$, diferenciando, obtemos

$$
\begin{aligned}
d x & =r\left(d b_{0}\right)_{g(x)} \circ d g(x) \\
d x & =r\left[\sum\left(\omega_{0}^{k}\right)_{g(x)}\left(b_{k}\right)_{g(x)}\right] \circ g(x) \\
d x & =r \sum g^{*}\left(\omega_{0}^{k}\right)_{g(x)}\left(b_{k}\right)_{g(x)}
\end{aligned}
$$

e logo $\left\{r g^{*} \omega_{0}^{0}, r g^{*} \omega_{0}^{1}, \ldots, r g^{*} \omega_{0}^{n}\right\}$ e $\left\{b_{0} \circ g, \ldots, b_{n} \circ g\right\}$ são bases duais. Denotando $\theta=$ $-i r g^{*} \omega_{0}^{0}$ e por $\theta^{j}=r g^{*} \omega_{0}^{j}$ poderemos escrever

$$
d x=\theta \otimes\left(i b_{0}\right)+\theta^{1} \otimes b_{1}+\cdots+\theta^{n} \otimes b_{n} .
$$

Diferenciando as formas $\theta$ e $\theta^{j}$ teremos

$$
\begin{aligned}
& d \theta=-i r g^{*}\left[d \omega_{0}^{0}\right]=-i r \sum g^{*} \omega_{0}^{l} \wedge g^{*} \bar{\omega}_{0}^{l}=-\frac{i}{r} \sum \theta^{l} \wedge \bar{\theta}^{l}, \\
& d \theta^{j}=r g^{*}\left[d \omega_{0}^{j}\right]=-r \sum\left(g^{*} \omega_{l}^{j}\right) \wedge\left(g^{*} \omega_{0}^{l}\right)=-\frac{1}{r} \sum \eta_{l}^{j} \wedge \theta^{l}-\frac{i}{r} \theta^{j} \wedge \theta, \\
& d \theta^{j}+\frac{1}{r} \sum\left(\eta_{l}^{j}-i \delta_{l}^{j} \theta\right) \wedge \theta^{l}=0, \quad \eta_{l}^{j}=r g^{*} \omega_{l}^{j} .
\end{aligned}
$$

Já sabemos que para as 1 -formas $\omega_{k}^{j}$

$$
d \omega_{k}^{j}+\sum \omega_{l}^{j} \wedge \omega_{k}^{l}=0
$$

Vejamos então qual é a expressão de $\Omega_{k}^{j}$ :

$$
\Omega_{k}^{j}=d \theta_{k}^{j}+\sum_{l=1}^{n} \theta_{l}^{j} \wedge \theta_{k}^{l},
$$

sendo $\theta_{l}^{j}=\frac{1}{r}\left(\eta_{l}^{j}-i \delta_{l}^{j} \theta\right), l, j=1,2, \ldots, n$ e $\theta_{0}^{j}=\frac{1}{r} \eta_{0}^{j}$.

$$
\begin{aligned}
& d \theta_{k}^{j}=\frac{1}{r}\left(d \eta_{k}^{j}-i \delta_{k}^{j} d \theta\right)=\frac{1}{r}\left(-\frac{1}{r} \sum_{l=0}^{n} \eta_{l}^{j} \wedge \eta_{k}^{l}-\frac{\delta_{k}^{j}}{r} \sum_{l=0}^{n} \theta^{l} \wedge \bar{\theta}^{l}\right), \\
& d \theta_{k}^{j}=-\frac{1}{r^{2}} \sum_{l=0}^{n}\left[\left(r \theta_{l}^{j}+i \delta_{l}^{j} \theta\right) \wedge\left(r \theta_{k}^{l}+i \delta_{k}^{l} \theta\right)+\delta_{k}^{j} \theta^{l} \wedge \bar{\theta}^{l}\right], \\
& d \theta_{k}^{j}+\sum_{l=1}^{n} \theta_{l}^{j} \wedge \theta_{k}^{l}=\theta_{0}^{j} \wedge \theta_{0}^{k}-\frac{\delta_{k}^{j}}{r^{2}} \sum_{l=1}^{n} \theta^{l} \wedge \bar{\theta}^{l}+\frac{i}{r^{2}} \sum_{l=1}^{n} \theta \wedge\left(\delta_{k}^{l} \theta_{l}^{j}-\delta_{l}^{j} \theta_{k}^{l}\right), \\
& d \theta_{k}^{j}+\sum \theta_{l}^{j} \wedge \theta_{k}^{l}=\frac{1}{r^{2}}\left[\theta^{j} \wedge \bar{\theta}^{k}-\delta_{k}^{j} \sum \theta^{l} \wedge \bar{\theta}^{l}\right] .
\end{aligned}
$$


ou em uma forma equivalente,

$$
\Omega_{j}^{k}=\frac{1}{r^{2}}\left(\theta^{k} \wedge \bar{\theta}^{j}-\delta_{j}^{k} \sum_{l=1}^{n} \theta^{l} \wedge \bar{\theta}^{l}\right) .
$$

Lembremos que todas as 1-formas que apareceram até agora são complexas.

Da forma como obtivemos $\left(x, b_{1}, \ldots, b_{n}\right)$ segue que $\left\{b_{1}(x), \ldots, b_{n}(x)\right\}$ é uma base de $\mathcal{D}_{x}$ e logo $\left\{\theta^{1}(x), \ldots, \theta^{n}(x)\right\}$ é base de $\mathcal{D}_{x}^{*}$. Podemos então definir $h=\sum_{j=1}^{n} \theta^{j} \otimes \bar{\theta}^{j}$ uma forma sesquilinear sobre $\mathcal{D}$. As $\theta_{j}^{k}$ nos dão uma boa derivação em $\mathcal{D}$, definida por

$$
\nabla b_{j}=\sum_{k=1}^{n} \theta_{j}^{k} \otimes b_{k} .
$$

É fácil verificar que $\nabla$ é compatível com $\langle\langle\rangle$,$\rangle (usando o isomorfismo \left.d \pi\right|_{\mathcal{D}}: \mathcal{D} \rightarrow T_{[z]} \mathbb{C P}^{n}$ ).

Como $\theta_{j}^{k}$ e $\theta^{j}$ são formas complexas, podemos escrevê-las da seguinte forma:

$$
\begin{aligned}
& \theta^{j}=\omega^{j}+i \omega^{j+n}, \\
& \theta_{k}^{j}=\omega_{k}^{j}+i \omega_{k}^{j+n}, \quad j=0,1, \ldots, n .
\end{aligned}
$$

Teremos então que

$$
\begin{aligned}
& d \omega^{j}+i d \omega^{j+n}+\sum\left(\omega_{k}^{j}+i \omega_{k}^{j+n}\right) \wedge\left(\omega^{k}+i \omega^{k+n}\right)=0 \\
& d \omega^{j}+\sum_{k=0}^{n} \omega_{k}^{j} \wedge \omega^{k}-\omega_{k}^{j+n} \wedge \omega^{k+n}=0 \\
& d \omega^{j+n}+\sum \omega_{k}^{j+n} \wedge \omega^{k}+\omega_{k}^{j} \wedge \omega^{k+n}=0
\end{aligned}
$$

que, usando $\omega_{k}^{j+n}=-\omega_{j+n}^{k}$, pode ser reescrita

$$
\begin{aligned}
& \left\{\begin{array}{l}
d \omega^{j}+\sum \omega_{k}^{j} \wedge \omega^{k}+\omega_{j+n}^{k} \wedge \omega^{k+n}=0, \\
d \omega^{j+n}+\sum \omega_{k}^{j+n} \wedge \omega^{k}+\omega_{k}^{j} \wedge \omega^{k+n}=0,
\end{array}\right. \\
& d \theta=-\frac{i}{r} \sum \theta^{j} \wedge \bar{\theta}^{j}=\frac{2}{r} \sum \omega^{j} \wedge \omega^{j+n} .
\end{aligned}
$$

Para todo $j, k=1, \ldots, n$ temos

$$
\begin{aligned}
\tilde{\Omega}_{k}^{j}= & \Omega_{k}^{j}+i \Omega_{k}^{j+n}=\frac{1}{r^{2}}\left\{\left(\omega^{j}+i \omega^{j+n}\right) \wedge\left(\omega^{k}-i \omega^{k+n}\right)\right. \\
& \left.+\delta_{k}^{j} \sum\left(\omega^{l}+\omega^{l+n}\right)\left(\omega^{l}-i \omega\right)\right\}, \\
\Omega_{k}^{j}= & \frac{1}{r^{2}}\left(\omega^{j} \wedge \omega^{k}+\omega^{j+n} \wedge \omega^{k+n}\right), \\
\Omega_{k}^{j+n}= & \frac{1}{r^{2}}\left\{\omega^{j+n} \wedge \omega^{k}-\omega^{j} \wedge \omega^{k+n}+\delta_{k}^{j} 2 \sum_{l=0}^{n} \omega^{l+n} \wedge \omega^{l}\right\} .
\end{aligned}
$$


Em $\mathcal{D}$ temos que todo vetor $X$ é da forma

$$
\begin{gathered}
X=X_{j} b_{j}, \quad b_{j}=f_{j}+i f_{j+n}, \\
\Omega_{j}^{j+n}=\frac{1}{r^{2}}\left\{2 \omega^{j+n} \wedge \omega^{j}+2 \sum_{l=0}^{n} \omega^{l+n} \wedge \omega^{l}\right\}, \\
\Omega_{j}^{j+n}=-\frac{4}{r^{2}} \omega^{j} \wedge \omega^{j+n}, \text { quando restrita a } a\left[b_{j}\right]
\end{gathered}
$$

isto é,

$$
\Omega_{j}^{j+n}\left(f^{j+n}, f^{j}\right)=\frac{4}{r^{2}} .
$$

Disto tiramos que as curvaturas seccionais de planos gerados por vetores da forma $u$ e $J u$ são constantes. A este tipo de plano denominamos planos holomorfos. Portanto, $\mathbb{C I P}^{n}$ possui curvatura dos planos holomorfos constante, resumidamente curvatura holomorfa constante.

\subsection{O espaço hiperbólico complexo}

O espaço hiperbólico complexo, $\mathbb{C H}^{n}\left(\frac{4}{r^{2}}\right)$, pode ser obtido de forma totalmente análoga a usada para construir o $\mathbb{C P}^{n}\left(\frac{4}{r^{2}}\right)$. De fato, consideremos em $\mathbb{C}^{n+1}$ o produto sesquilinear $h$

$$
h(z, w)=z_{0} \bar{w}_{0}-z_{1} \bar{w}_{1}-\cdots-z_{n} \bar{w}_{n}
$$

definamos a seguinte hipervariedade real do $\mathbb{C}^{n+1}$

$$
Q_{r}^{2 n+1}=\left\{z \in \mathbb{C}^{n+1}: h(z, z)=r^{2}\right\}
$$

É fácil verificar que isto realmente é uma hipervariedade. $R: Q^{2 n+1} \times S^{1} \longrightarrow Q^{2 n+1}$ dada por

$$
(z, \lambda) \stackrel{R}{\longrightarrow} \lambda z
$$

é uma ação $\mathcal{C}^{\infty}$ do $S^{1}$ em $Q^{2 n+1}$. Em $Q^{2 n+1}$ definimos a seguinte relação de equivalência $\sim: z \sim z^{\prime}$ se existe $\lambda \in S^{1}$ tal que $z^{\prime}=\lambda z$. Definimos então

$$
\mathbb{C H}^{n}\left(\frac{4}{r^{2}}\right)=Q_{r}^{2 n+1} / \sim
$$

denotando as classe de equivalência de $z$ por $[z]$ teremos $\mathbb{C H}^{n}\left(\frac{4}{r^{2}}\right)=\left\{[z]: z \in Q_{r}^{2 n+1}\right.$. $\mathbb{C H}^{n}\left(\frac{4}{r^{2}}\right)$ possui uma topologia induzida pela projeçào $\pi: Q_{r}^{2 n+1} \longrightarrow \mathbb{C H}^{n}\left(\frac{4}{r^{2}}\right)$ dada por 
$\pi(z)=[z]$. A estrutura de variedade diferenciável sobre $\mathbb{C H}^{n}\left(\frac{4}{r^{2}}\right)$ é obtida definindo a carta $(\psi, U)$ da seguinte forma

$$
\begin{aligned}
& U=\mathbb{C H}^{n}\left(\frac{4}{r^{2}}\right) \\
& \psi([z])=\frac{1}{z_{0}}\left(z_{1}, \ldots, z_{n}\right)
\end{aligned}
$$

temos assim um recobrimento de $\mathbb{C}^{n}\left(\frac{4}{r^{2}}\right)$ por abertos, é suficiente então pegar a família maximal holomorficamente compatível com este atlas. Repetindo todos os passos feitos para o $\mathbb{C P}^{n}$ termos que $\left(Q_{r}^{2 n+1}, \mathbb{C} \mathbb{H}^{n}\left(\frac{4}{r^{2}}\right), S^{1}, \pi\right)$ é um fibrado principal com espaço total $Q^{2 n+1}$, espaço base $\mathbb{C} \mathbb{H}^{n}$ e fibra $S^{1}$. O espaço tangente de $Q_{r}^{2 n+1}$ em $z$ é dado por

$$
T_{z} Q_{r}^{2 n+1}=\left\{v \in \mathbb{C}^{n+1}: h(z, v)+\overline{h(v, z)}=0\right\} .
$$

Também verifica-se que $\xi_{z}=\frac{i z}{r}$ pertence ao espaço tangente de $H^{2 n+1}$ em $z$. $\left.d \pi\right|_{\mathcal{D}}$ é um isomorfismo entre $\mathcal{D}_{z}$ e o espaço tangente em $z$ do espaço hiperbólico complexo que nos possibilitará munir $\mathbb{C H}^{n}\left(\frac{4}{r^{2}}\right)$ de uma métrica $\langle\langle\rangle$,$\rangle . Em verdade, como \left.b\right|_{\text {cald } \times \mathcal{D}}$ é negativa definida é suficiente defini-la por

$$
\left\langle\left\langle d \pi_{\gamma(t)}\left(\gamma^{\prime}(t)\right), d \pi_{\gamma(t)}\left(\gamma^{\prime}(t)\right)\right\rangle\right\rangle=r^{2} \frac{h(\tilde{\gamma}(t), \tilde{\gamma}(t)) h\left(\tilde{\gamma}^{\prime}(t), \tilde{\gamma}^{\prime}(t)\right)-\left|h\left(\tilde{\gamma}(t), \tilde{\gamma}^{\prime}(t)\right)\right|^{2}}{h(\tilde{\gamma}(t), \tilde{\gamma}(t))^{2}} .
$$

Formalmente, podemos escrever

$$
\pi^{*}\left\langle\langle,\rangle=r^{2} \frac{h(z \wedge d z, z \wedge d z)}{h(z, z)^{2}} .\right.
$$

De forma análoga ao $\mathbb{C P}^{n}\left(\frac{4}{r^{2}}\right)$, pode-se demonstrar que o $\mathbb{C H}^{n}\left(\frac{4}{r^{2}}\right)$ possui curvaturas holomorfas constantes e negativas. 


\section{Geometria sub-Riemanniana}

\subsection{Definições e a conexão sub-Riemanniana}

Agora que já definimos e obtivemos alguns resultados dos espaços nos quais iremos trabalhar, começaremos as definições dos objetos que "habitarão" esses espaços e a busca de alguns de seus principais resultados. Para tal, façamos algumas definições.

Definição 2.1.1 Seja $M$ uma variedade diferenciável de dimensão n e TM o fibrado tangente de $M$. A um conjunto $\mathcal{D}=\cup_{p \in M} \mathcal{D}_{p}$, sendo $\mathcal{D}_{p}$ um subespaço vetorial de $T_{p} M$, denominamos de distribuição. Se a co-dimensão de $\mathcal{D}_{p}$ for constante e igual a $k$ dizemos que $\mathcal{D}$ possui co-dimensão $k$. Dizemos que $\mathcal{D}$ é de classe $\mathcal{C}^{\infty}$ se para todo $p \in M$ existem uma vizinhança $U$ de $p$ e $k$ 1-formas $\left\{\theta^{1}, \theta^{2}, \ldots, \theta^{k}\right\}$ de classe $\mathcal{C}^{\infty}$ em $U$ tal que $\mathcal{D}_{x}=$ $\cap_{j=1}^{k} k \operatorname{er} \theta_{x}^{j}$ para todo $x \in U$.

Quase sempre uma definição em $T M$ nos leva a uma definição no fibrado co-tangente $T^{*} M$.

Definição 2.1.2 Seja $M$ uma variedade diferenciável de dimensão n e $T^{*} M$ o fibrado co-tangente de $M$. A um conjunto $\mathcal{D}^{*}=\cup_{p \in M} \mathcal{D}_{p}^{*}$, sendo $\mathcal{D}_{p}^{*}$ um subespaço vetorial do dual de $T_{p} M$, denominamos de co-distribuição. Se a dimensão de $\mathcal{D}_{p}^{*}$ for constante e igual a $k$ dizemos que $\mathcal{D}^{*}$ possui dimensão $k$. Dizemos que $\mathcal{D}$ é de classe $\mathcal{C}^{\infty}$ se para todo $p \in M$ existem uma vizihança $U$ de $p$ e $n-k$ campos linearmente independentes e de classe $\mathcal{C}^{\infty}$ em $U \quad\left\{X^{1}, X^{2}, \ldots, X^{n-k}\right\}$ tal que $\mathcal{D}_{x}^{*}=\left\{\omega \in T_{x} M^{*} \quad: \quad \omega\left(X_{j}\right)=0, \quad j=1, \ldots, n-k\right\}$.

Definição 2.1.3 Uma variedade sub-Riemanniana consiste em uma terna $(M, \mathcal{D}, g)$, sendo $M$ uma variedade diferenciável, $\mathcal{D}$ uma distribuição $\mathcal{C}^{\infty}$ de co-dimensão $k$ e g uma 
métrica definida sobre $\mathcal{D}$ e tal que, para quaisquer dois campos $X$ e $Y\left(\mathcal{C}^{\infty}\right)$ em $\mathcal{D}$, $g(X, Y)$ é uma função $\mathcal{C}^{\infty}$.

Observe que no caso em que $[\mathcal{D}, \mathcal{D}] \subset \mathcal{D}(\mathcal{D}$ integrável) teremos um decomposição de $M$ em subvariedades integrais maximais de $\mathcal{D}$ ( folhas, como são comumente denominadas ). Em [21] verifica-se que é possível demonstrar que dotando o conjunto das distribuições $\mathcal{C}^{\infty}$ de uma certa topologia o conjunto das distribuições integráveis será magro.

Definição 2.1.4 Uma distribuição $\mathcal{D}$ em uma variedade diferenciável $M$ é dita completamente não integrável se TM pode ser obtido pela soma de $\mathcal{D}$ com seus colchetes, isto é, $T M=\mathcal{D}+[\mathcal{D}, \mathcal{D}]+[[\mathcal{D}, \mathcal{D}], \mathcal{D}]+\cdots$.

O caso que realmente nos interessa neste trabalho é o obtido quando $\mathcal{D}$ é não integrável, em especial o de co-dimensão um, isto é, $T M=\mathcal{D}+[\mathcal{D}, \mathcal{D}]$.

Definição 2.1.5 Uma curva de classe $\mathcal{C}^{\infty} \gamma:[0,1] \longrightarrow M$ é dita admissível se o vetor tangente $\dot{\gamma}(t)$ pertence a distribuição $\mathcal{D}$ para todo $t \in[0,1]$.

Enunciaremos abaixo, sem demonstração, um teorema muito importante para a geometria sub-Riemanniana. Esse teorema é a "luz" em muitos problemas de teoria de controle e otimização. Alguns autores atribuem esse teorema ao matemático W. L. Chow enquanto outros a W. L. Chow e P. A. Rashvskii.

Teorema 2.1.6 Para quaisquer dois pontos p e q em uma variedade sub-Riemanniana $(M, \mathcal{D}, g)$ com uma distribuição completamente não integrável $\mathcal{D}$ existe um curva admissível $\mathcal{C}^{\infty}$ por partes $\gamma$ ligando $p$ a $q$, isto é, $\gamma(0)=p$ e $\gamma(1)=q$.

Em nosso trabalho estaremos interessados em um tipo particular de variedade subRiemanniana: as que possuem $\mathcal{D}$ de codimensão 1 e um campo $\xi$ globalmente definido e tal que $T_{p} M=\mathcal{D}_{p} \oplus \xi_{p}$, para todo $p \in M$. Neste caso $g$ pode ser estendida a uma métrica $\tilde{g}$ definida em todo espaço tangente de $M$, na maior parte das vezes denotada por $\langle$,$\rangle , isto$ é feito definindo $\tilde{g}: T M \times T M \rightarrow \mathbb{R}$ bilinear tal que $\left.\tilde{g}\right|_{\mathcal{D} \times \mathcal{D}}=g, \tilde{g}(\xi, \xi)=1$ e $\tilde{g}(\xi, X)=0$ se $X \in \mathcal{D}$. Podemos então definir uma 1-forma tal que $\theta(X)=\tilde{g}(\xi, X)$. Desta definição tiramos que $\mathcal{D}=\operatorname{ker} \theta$ e $\theta(\xi)=1$. $\mathcal{D}$ recebe algumas denominações conforme a forma bilinear $\left.d \theta\right|_{\mathcal{D}}$ seja não-degenerada ou definida ou nula. Dizemos que $\mathcal{D}$ é de contato se $\theta \wedge d \theta$ é não-degenerada, $\mathcal{D}$ é fortemente pseudo-convexa se $d \theta$ restrita a $\mathcal{D}$ é definida e $\mathcal{D}$ 
será integrável se $d \theta \equiv 0$ quado restrita a $\mathcal{D}$. Neste último, $M$ é uma variedade folheada. Temos, como em geometria Riemanniana, uma conexão associada à estrutura $(M, \mathcal{D}, g, \xi)$. A existência e unicidade da conexão adaptada é provada no teorema abaixo.

Teorema 2.1.7 Existe uma única conexão $\nabla$ sobre $(M, \mathcal{D}, \xi, g)$ com as seguintes propriedades:

(i) $\nabla_{X} Y \in \mathcal{D}$ para todas $X, Y \in \mathcal{D}$;

(ii) $\nabla_{X} \xi=0$ para todo $X \in \mathscr{X}(M)$;

(iii) $\nabla_{X} g=0$ para todo $X \in \mathscr{X}(M)$;

(iv) $T(X, Y)=d \theta(X, Y) \xi$ para todo $X, Y \in \mathcal{D}$;

(v) $T(\xi, X)=\tau(X)+d \theta(\xi, X) \xi$ para todo $X \in \mathcal{D}$.

Demonstração: Seja $\left\{e_{1} ; \ldots ; e_{n} ; \xi\right\}$ um referencial ortonormal (local) em $M$. Podemos então escrever

$$
\begin{aligned}
{\left[e_{i}, e_{j}\right] } & =\sum_{k=1}^{n} C_{i j}^{k} e_{k}+d_{i j} \xi \\
{\left[\xi, e_{j}\right] } & =\sum_{k=1}^{n} C_{j}^{k} e_{k}+d_{j} \xi
\end{aligned}
$$

Sendo $\nabla$ a conexão, poderemos escrever

$$
\begin{aligned}
\nabla_{e_{i}} e_{j} & =\sum_{k} \Gamma_{i j}^{k} e_{k}, \\
\nabla_{e_{i}} \xi & =0 \\
\nabla_{\xi} e_{j} & =\operatorname{sum}_{k} \Gamma_{j}^{k} e_{k} .
\end{aligned}
$$

$\operatorname{De} T\left(e_{i}, e_{j}\right)=\nabla_{e i} e_{j}-\nabla_{e_{j}} e_{i}-\left[e_{i}, e_{j}\right]$ e de (iv) tiramos que $d \theta\left(e_{i}, e_{j}\right) \xi=\left(\Gamma_{i j}^{k}-\Gamma_{j i}^{k}-C_{i j}^{k}\right) e_{k}-$ $d_{i j} \xi$ e portanto $C_{i j}^{k}=\Gamma_{i j}^{k}-\Gamma_{j i}^{k}$. De (iii) obtemos que $\Gamma_{i j}^{k}+\Gamma_{i k}^{j}=0$. Conseqüentemente, teremos $\Gamma_{i j}^{k}=\frac{1}{2}\left(C_{i j}^{k}+C_{k 1}^{j}-C_{j k}^{i}\right)$. Isto prova a existência e unicidade de (i), (ii), (iii) e parte de (iv) e (v). Para determinarmos $\tau$ procederemos da mesma maneira.

$$
T\left(\xi, e_{j}\right)=\nabla_{\xi} e_{j}-\left[\xi, e_{j}\right]=\left(\Gamma_{j}^{k}-C_{j}^{k}\right) e_{k}-d_{j} \xi
$$

e portanto escrevendo $T\left(\xi, e_{j}\right)=\tau_{j}^{k} e_{k}+d_{j} \xi$ teremos $\tau_{k}^{j}=\Gamma_{j}^{k}-C_{j}^{k}$. Usando que $d \theta(\xi, X)=$ $-\theta([\xi, X])$ tiramos que $d_{j}=-d \theta(\xi, X)$ para todo $X \in \mathcal{D}$ Novamente, de (iii), tiramos 
que $\Gamma_{k}^{j}+\Gamma_{j}^{k}=0$. Da simetria de $\tau$ tiramos que $\tau_{j}^{k}=\tau_{k}^{j}$ e portanto $\Gamma_{j}^{k}=\frac{1}{2}\left(C_{j}^{k}-C_{k}^{j}\right)$ e $\tau_{j}^{k}=-\frac{1}{2}\left(C_{j}^{k}+C_{k}^{j}\right)$.

\subsection{Propriedades da conexão sub-Riemanniana}

Examinemos algumas propriedades da conexão adaptada à estrutura sub-Riemanniana de $M$. Abaixo, dada uma 2 -forma $\omega$, usamos $i_{\xi} \omega$ para denotar a um forma $i_{\xi} \omega(X)=\omega(\xi, X)$.

Proposição 2.2.1 Se $\xi$ é tal que $i_{\xi} d \theta=0$, então a derivada de Lie de $\theta$ na direção de $\xi$ é nula $\left(\mathcal{L}_{\xi} \theta=0\right)$.

Demonstração: sabemos que em relação a métrica $\theta$ se escreve como $\theta(X)=\langle X, \xi\rangle$. Daí temos

$$
\begin{aligned}
\left(\mathcal{L}_{\xi} \theta\right)(X) & =\mathcal{L}_{\xi} \theta(X)-\theta\left(\mathcal{L}_{\xi} X\right) \\
& =\left\langle\nabla_{\xi} X, \xi\right\rangle-\left\langle\mathcal{L}_{\xi} X, \xi\right\rangle \\
& =\left\langle\nabla_{\xi} X-[\xi, X], \xi\right\rangle \\
& =0
\end{aligned}
$$

Corolário 2.2.2 Se $\mathcal{D}$ e de contato, então $\mathcal{L}_{\xi} \theta(\mathcal{D}) \subset \mathcal{D}$.

Demonstração: se $X \in \mathcal{D}$, da proposição anterior

$$
\begin{aligned}
\theta\left(\mathcal{L}_{\xi} X\right) & =\mathcal{L}_{\xi} \theta(X) \\
\left\langle\mathcal{L}_{\xi} X, \xi\right\rangle & =0
\end{aligned}
$$

Proposição 2.2.3 Para campos $\mathcal{C}^{\infty} X$ e $Y$ em $\mathcal{D}$ temos $\langle\tau(X), Y\rangle=\frac{1}{2}\left(\mathcal{L}_{\xi} g\right)(X, Y)$. 
Demonstração: da substituição de $\tau(X)=\nabla_{\xi} X-[\xi, X]$ em $g(\tau(X), Y)$

$$
\begin{aligned}
g(\tau(x), Y) & =g\left(\nabla_{\xi} X, Y\right)-g([\xi, X], Y) \\
& =\xi g(X, Y)-g\left(X, \nabla_{\xi} Y\right)+\xi g(X, Y)+g(X,[\xi, Y])+\left(\mathcal{L}_{\xi} g\right)(X, Y) \\
& =-g(X, \tau(Y))+\left(\mathcal{L}_{\xi} g\right)(X, Y) \\
& =-g(\tau(X), Y)+\left(\mathcal{L}_{\xi} g\right)(X, Y)
\end{aligned}
$$

e portanto segue que $\langle\tau(X), Y\rangle=\frac{1}{2}\left(\mathcal{L}_{\xi} g\right)(X, Y)$.

Corolário 2.2.4 Se $\xi$ é um campo de Killing para g então $\tau$ é nula.

Em geral, para uma variedade sub-Riemanniana de co-dimensão 1 com um $\xi$ globalmente definido e transversalmente orientável, verifica-se que existe um campo tensorial $\tilde{h}$ de tipo $(1,1)$ tal que

$$
d \theta(X, Y)=\langle\widetilde{h}(X), Y\rangle \text { para campos } X, Y \in \mathcal{X}(\mathcal{M})
$$

e podemos definir o operador $h$ da seguinte forma:

$$
h(X)=\tilde{h}(X)-\langle X, \xi\rangle \xi
$$

Portanto, podemos calcular a derivada covariante sub-Riemanniana de $d \theta$ na direção $\xi$

$$
\begin{aligned}
\left(\nabla_{\xi} d \theta\right)(X, Y) & =\xi d \theta(X, Y)-d \theta\left(\nabla_{\xi} X, Y\right)-d \theta\left(X, \nabla_{\xi} Y\right) \\
& =g\left(\nabla_{\xi} \widetilde{h}(X), Y\right)+g\left(\widetilde{h}(X), \nabla_{\xi} Y-g\left(\widetilde{h}\left(\nabla_{\xi} X\right), Y\right)-g\left(\widetilde{h}(X), \nabla_{\xi} Y\right.\right. \\
& =g\left(\left(\nabla_{\xi} \widetilde{h}\right)(X)+\widetilde{h}\left(\nabla_{\xi} X\right), Y\right)-g\left(\widetilde{h}\left(\nabla_{\xi} X\right), Y\right)-g\left(\widetilde{h}(X), \nabla_{\xi} Y\right) \\
& =g\left(\left(\nabla_{\xi} \widetilde{h}\right)(X), Y\right\rangle .
\end{aligned}
$$

Proposição 2.2.5 Se $\tau$ é paralelo, ao longo do fluxo de $\xi$, em relação à conexão subRiemanniana $\nabla$, então $\mathcal{L}_{\xi} \nabla_{\xi} X=\nabla_{\xi} \mathcal{L}_{\xi} X$ para todo $X$ em $\mathcal{D}$.

Demonstração: sabemos que

$$
\begin{aligned}
\left(\nabla_{\xi} \tau\right)(X) & =\nabla_{\xi} \mathcal{T}(X)-\tau\left(\nabla_{\xi} X\right) \\
& =\nabla_{\xi} \nabla_{\xi} X-\nabla_{\xi} \mathcal{L}_{\xi}(X)-\nabla_{\xi} \nabla_{\xi} X-\mathcal{L}_{\xi} \nabla_{\xi} X \\
& =\left(\mathcal{L}_{\xi} \nabla_{\xi} X-\nabla_{\xi} \mathcal{L}_{\xi}\right)(X) .
\end{aligned}
$$


Definição 2.2.6 Dadas duas variedades sub-Riemannianas $(M, \mathcal{D}, g, \xi)$ e $(\widehat{M}, \widehat{\mathcal{D}}, \hat{g}, \hat{\xi})$ e uma aplicação diferenciável $f: M \longrightarrow \widehat{M}$ dizemos que fé uma isometria sub-Riemanniana local se:

i) $d f(\mathcal{D})=\widehat{\mathcal{D}}$;

ii) $\hat{g}(d f(X), d f(Y))=g(X, Y)$;

iii) $d f(\xi)=\hat{\xi}$;

para campos $X$ e $Y$ em $\mathcal{D}$.

Definição 2.2.7 Uma variedade sub-Riemanniana $(M, \mathcal{D}, g, \xi)$ é dita homogênea se o conjunto das isometrias sub-riemannianas de $M$ age transitivamente em $M$.

\subsection{Exemplos de variedades sub-Riemannianas ho- mogêneas}

Começaremos os exemplos de variedades sub-Riemannianas homogêneas com as variedades homogêneas mais simples: os grupos de Lie de dimensão 3 com uma métrica (sub-Riemanniana) invariante à esquerda.

\section{Grupos de Lie Tridimensionais}

Seja $G$ um grupo de Lie de dimensão 3 , g sua álgebra de Lie associada e $\mathcal{D}$ um subespaço de $\mathfrak{g}$ de dimensão 2. Sendo $\left\{e_{1} ; e_{2}\right\}$ uma base de $\mathcal{D}$, assumamos que $\left[e_{1}, e_{2}\right] \notin \mathcal{D}$. Poderemos, portanto, definir uma métrica em $\mathcal{D}$ invariante à esquerda sobre o $G$ impondo que $e_{1}$ e $e_{2}$ são ortonormais. Teremos assim a terna $(G, \mathcal{D}, g)$ sendo uma estrutura sub-Riemanniana sobre $G$. Se $\left\{\theta^{1} ; \theta^{2} ; \theta^{3}\right\}$ for a base dual à base $\left\{e_{1} ; e_{2} ;\left[e_{1}, e_{2}\right]\right\}$, o elemento de área será dado por $d A=\theta^{1} \wedge \theta^{2}$ e $\left.d \theta^{3}\right|_{\mathcal{D}}=\lambda \theta^{1} \wedge \theta^{2}$ para alguma constante $\lambda \neq 0 \in \mathbb{R}$.

Poderemos então definir $\theta=\frac{2}{\lambda} \theta^{3}$. Deste modo teremos $\operatorname{ker} \theta=\mathcal{D}$ e $d \theta=2 \theta^{1} \wedge \theta^{2}$, isto é, $\theta$ é o que denomina-se uma forma de contato. Não é difícil mostrar que existe um campo $\xi$ tal que $\theta(\xi)=1$ e $i_{\xi} d \theta=0$. Tomando como base de $\mathfrak{g}\left\{e_{1} ; e_{2} ; \xi\right\}$ teremos para os colchetes as seguintes expressões:

$$
\left[e_{1}, e_{2}\right]=\Gamma_{1} e_{1}+\Gamma_{2} e_{2}-2 \xi
$$


2.3 Exemplos de variedades sub-Riemannianas homogêneas

$$
\begin{aligned}
& {\left[e_{2}, \xi\right]=\Gamma e_{1}+\lambda e_{2},} \\
& {\left[\xi, e_{1}\right]=\lambda e_{1}+\Upsilon e_{2} .}
\end{aligned}
$$

Fazendo uso da conexão associada à distribuição $\mathcal{D}$, poderemos escrever as equações de estrutura para este exemplo:

$$
\begin{aligned}
\nabla e_{1} & =\omega \otimes e_{2}, \\
\nabla e_{2} & =-\omega \otimes e_{1}, \\
\nabla \xi & =0 .
\end{aligned}
$$

Para o co-referencial $\left\{\theta^{1} ; \theta^{2} ; \theta\right\}$ teremos

$$
\begin{aligned}
\nabla \theta^{1} & =\omega \otimes \theta^{2}, \\
\nabla \theta^{2} & =-\omega \otimes \theta^{1}, \\
\nabla \theta & =0,
\end{aligned}
$$

e portanto em relação a esta base teremos

$$
\begin{aligned}
d \theta^{1} & =\omega \wedge \theta^{2}+\tau^{1} \wedge \theta, \\
d \theta^{2} & =-\omega \wedge \theta^{1}+\tau^{2} \wedge \theta, \\
d \theta & =2 \theta^{1} \wedge \theta^{2} .
\end{aligned}
$$

Mas, olhando para (2.1) teremos que

$$
\left\{\begin{array}{l}
d \theta^{1}=-\Gamma_{1} \theta^{1} \wedge \theta^{2}-\Gamma \theta^{2} \wedge \theta+\lambda \theta^{1} \wedge \theta \\
d \theta^{2}=-\Gamma_{2} \theta^{1} \wedge \theta^{2}-\lambda \theta^{2} \wedge \theta+\Upsilon \theta^{1} \wedge \theta,
\end{array}\right.
$$

e um processo de anti-simetrização em $\theta^{1}$ e $\theta^{2}$ nos leva a

$$
\left\{\begin{array}{l}
d \theta^{1}=\left(-\Gamma_{1} \theta^{1}-\Gamma_{2} \theta^{2}+\frac{\Upsilon+\Gamma}{2} \theta\right) \wedge \theta^{2}+\left(\lambda \theta^{1}+\frac{\Upsilon-\Gamma}{2} \theta^{2}\right) \wedge \theta \\
d \theta^{2}=\left(\Gamma_{1} \theta^{1}+\Gamma_{2} \theta^{2}-\frac{\Upsilon+\Gamma}{2} \theta\right) \wedge \theta^{1}+\left(\frac{\Upsilon-\Gamma}{2} \lambda \theta^{2}\right) \wedge \theta,
\end{array}\right.
$$

isto é,

$$
\begin{aligned}
\omega & =-\Gamma_{1} \theta^{1}-\Gamma_{2} \theta^{2}+\frac{\Upsilon+\Gamma}{2} \theta, \\
\tau^{1} & =\lambda \theta^{1}+\frac{\Upsilon-\Gamma}{2} \theta^{2}, \\
\tau^{2} & =\frac{\Upsilon-\Gamma}{2} \theta^{1}-\lambda \theta^{2},
\end{aligned}
$$


e um cálculo simples nos dará

$$
\begin{aligned}
d \omega & =\left(\Gamma_{1}^{2}+\Gamma_{2}^{2}+\Upsilon+\Gamma\right) \theta^{1} \wedge \theta^{2}-\left(\lambda \Gamma_{1}+\Upsilon \Gamma_{2}\right) \theta^{1} \wedge \theta+\left(\Gamma_{1} \Gamma+\Gamma_{2} \lambda\right) \theta^{2} \wedge \theta, \\
K & =-\left(\Gamma_{1}^{2}+\Gamma_{2}^{2}+\Upsilon+\Gamma\right), \\
w_{1} & =\lambda \Gamma_{1}+\Upsilon \Gamma_{2}, \\
w_{2} & =\Gamma_{1} \Gamma+\lambda \Gamma_{2} .
\end{aligned}
$$

$K$ é denominado curvatura seccional sub-Riemanniana.

Para uma melhor análise dos grupos de Lie tridimensionais necessitamos de algumas definições.

Definição 2.3.1 Um grupo de Lie G será denominado unimodular se qualquer forma volume invariante à esquerda também for invariante à direita.

Lembremos que $A d: G \rightarrow \operatorname{Aut}(g)$ é definida como $A d(g)=d\left(R_{g^{-1}} \circ L_{g}\right)_{e}$, sendo $L_{g}\left(R_{g}\right)$ a translação à esquerda (direita).

Lema 2.3.2 O grupo $G$ é unimodular se e somente se $\operatorname{det}[A d(g)]= \pm 1$, para todo $g \in G$.

Demonstração: Seja $\omega$ uma forma volume sobre $G$. Suponhamos que $L_{g}^{*} \omega=\omega, \forall g \in G$. De

$$
A d(g)=d\left(R_{g^{-1}} \circ L_{g}\right)_{e}
$$

tiramos que

$$
\begin{aligned}
\left(d R_{g}\right)_{g^{-1}} & =\operatorname{Ad}\left(g^{-1}\right)\left(d L_{g}\right)_{g^{-1}} \\
R_{g}^{*} \omega_{g}\left(u_{1}, u_{2}, u_{3}\right) & =\omega_{g}\left(\left(d R_{g}\right)_{g^{-1}}\left(u_{1}\right),\left(d R_{g}\right)_{g^{-1}}\left(u_{2}\right),\left(d R_{g}\right)_{g^{-1}}\left(u_{3}\right)\right) \\
& \left.=\mid \operatorname{det} A d\left(g^{-1}\right)\right) \mid \omega_{g}\left(\left(d L_{g}\right)_{g^{-1}}\left(u_{1}\right),\left(d L_{g}\right)_{g^{-1}}\left(u_{2}\right), \omega_{g}\left(\left(d L_{g}\right)_{g^{-1}}\left(u_{3}\right)\right)\right. \\
& =\left|\operatorname{det} A d\left(g^{-1}\right)\right| L_{g}^{*} \omega_{g}\left(u_{1}, u_{2}, u_{3}\right) \\
& =\left|\operatorname{det} A d\left(g^{-1}\right)\right| \omega_{e}\left(u_{1}, u_{2}, u_{3}\right) .
\end{aligned}
$$

portanto, se $\mathrm{G}$ for unimodular, da igualdade acima temos que $\operatorname{det} A d(g)= \pm 1$. E da igualdade acima temos que a reciproca também é verdadeira. 
Lema 2.3.3 Um grupo de Lie conexo é unimodular se e somente se a aplicação ad $(X)$ : $\mathfrak{g} \rightarrow \mathfrak{g}$, dada por ad $(X)(Y)=[X, Y]$, possui traço nulo.

Demonstração: Sabemos que $\exp \mathfrak{g} \rightarrow G$ é $\mathcal{C}^{\infty}$ e que

$$
\begin{aligned}
A d(\exp (X)) & =\sum_{k=0}^{\infty} \frac{\operatorname{ad}(X)^{k}}{k !} \\
\operatorname{det}(\operatorname{ad}(\exp (X))) & =\sum_{k=0}^{+\infty} \frac{[\operatorname{tr}(\operatorname{ad}(X))]^{k}}{k !} .
\end{aligned}
$$

Se $\operatorname{tr}(a d(X))=0$ para todo $X \in \mathfrak{g}$, então $\operatorname{det}(A d(g))=1$ em uma vizinhança $V$ de $e$ e portanto como $G=\bigcup_{n=0}^{\infty} V^{n}$, segue que $\operatorname{det}(A d(h))=1$, para todo $h \in G$. Reciprocamente, se $\operatorname{det}(A d(g))=1$, para todo $G$, então

$$
1=\sum_{k=0}^{\infty} \frac{\operatorname{tr}(\operatorname{ad}(X))]^{k}}{k !} \quad \text { implica em } \operatorname{tr}[\operatorname{ad}(X)]=0, \quad \forall X \in \mathfrak{g} .
$$

Definição 2.3.4 g é denominada uma álgebra de Lie unimodular se $\operatorname{tr}[\operatorname{ad}(X)]=0$ para todo $X \in \mathfrak{g}$.

Para prosseguirmos no exemplo tridimensional, dividiremos o estudo em dois casos: $G$ unimodular e $G$ não-unimodular e usaremos algumas idéias e definições de [8] .

Lema 2.3.5 $\mathcal{U}=\{X \in \mathfrak{g} \mid \operatorname{tr}[\operatorname{ad}(X)]=0\}$ é um ideal em $\mathfrak{g}$.

Demonstração: De $\operatorname{ad}[X, Y]=\operatorname{ad}(X) \circ \operatorname{ad}(Y)-\operatorname{ad}(Y) \circ \operatorname{ad}(X)$ e de $\operatorname{tr}[\operatorname{ad}(X) \circ \operatorname{ad}(Y)]=$ $\operatorname{tr}[\operatorname{ad}(Y)$ o $\operatorname{ad}(X)]$ segue que $\mathfrak{g}^{1}=[\{[X, Y] \mid X, Y \in \mathfrak{g}\}] \subset \mathcal{U}$ e portanto $\mathcal{U}$ é um ideal.

Definição 2.3.6 $\mathcal{U}$ é denominado o ideal unimodular de $G$.

Se $\mathfrak{g}$ é a álgebra de Lie de $G(\operatorname{dim} G=3)$ podemos, fixado um produto interno em $\mathfrak{g}$ e uma orientação, definir um produto vetorial $\wedge$ em $\mathfrak{g}$ por

$$
\langle u \wedge v, w\rangle=\operatorname{det}(u, v, w)
$$


Lema 2.3.7 $O$ colchete [, ] está relacionado com $\wedge$ pela fórmula $[u, v]=L(u \wedge v)$, sendo $L$ uma aplicação linear de $\mathfrak{g}$ em $\mathfrak{g}$ definida univocamente. O grupo de Lie $G$ será unimodular se e somente se $L$ é auto-adjunta $\left(L=L^{t}\right)$.

Demonstração: escolhamos $\left\{e_{1} ; e_{2} ; e_{3}\right\}$ como sendo uma base ortonormal de g. Definamos $L$, como em [8] , uma aplicação linear tal que

$$
\begin{aligned}
& L\left(e_{1}\right)=\left[e_{2}, e_{3}\right], \\
& L\left(e_{2}\right)=\left[e_{3}, e_{1}\right], \\
& L\left(e_{3}\right)=\left[e_{1}, e_{2}\right] .
\end{aligned}
$$

Se $X^{j}=X_{1}^{j} e_{1}+X_{2}^{j} e_{2}+X_{3}^{j} e_{3}, j=1,2$,

$$
\begin{aligned}
L\left(X^{1} \wedge X^{2}\right)= & L\left[\left(X_{2}^{1} X_{3}^{2}-X_{2}^{2} X_{3}^{1}\right) e_{1}+\left(X_{3}^{1} X_{1}^{2}-X_{3}^{2} X_{1}^{1}\right) e_{2}+\left(X_{1}^{1} X_{2}^{2}-X_{1}^{2} X_{2}^{1}\right) e_{3}\right] \\
= & \left(X_{2}^{1} X_{3}^{2}-X_{3}^{1} X_{2}^{1}\right)\left[e_{2}, e_{3}\right]+\left(X_{3}^{1} X_{1}^{2}-X_{1}^{1} X_{3}^{2}\right)\left[e_{3}, e_{1}\right] \\
& +\left(X_{1}^{1} X_{2}^{2}-X_{2}^{1} X_{1}^{2}\right)\left[e_{1}, e_{2}\right] \\
= & {\left[X^{1}, X^{2}\right] . }
\end{aligned}
$$

Escrevendo a matriz de $L$ em relação à base $\left\{e_{1} ; e_{2} ; e_{3}\right\}$, teremos

$$
\begin{gathered}
L\left(e_{j}\right)=\sum_{i=1}^{3} L_{j}^{i} e_{i} \\
\operatorname{ad}\left(e_{1}\right)\left(e_{1}\right)=0 e_{1}+0 e_{2}+0 e_{3}, \\
\operatorname{ad}\left(e_{1}\right)\left(e_{2}\right)=\left[e_{1}, e_{2}\right]=L\left(e_{3}\right)=L_{3}^{1} e_{1}+L_{3}^{2} e_{2}+L_{3}^{3} e_{3}, \\
\operatorname{ad}\left(e_{1}\right)\left(e_{3}\right)=\left[e_{1}, e_{3}\right]=-L\left(e_{2}\right)-L_{2}^{1} e_{1}-L_{2}^{1} e_{2}-L_{2}^{3} e_{3}, \\
\operatorname{tr}\left[\operatorname{ad}\left(e_{1}\right)\right]=L_{3}^{2}-L_{2}^{3} .
\end{gathered}
$$

De forma análoga,

$$
\begin{aligned}
& \operatorname{tr}\left[\operatorname{ad}\left(e_{2}\right)\right]=L_{1}^{3}-L_{3}^{1}, \\
& \operatorname{tr}\left[\operatorname{ad}\left(e_{3}\right)\right]=L_{2}^{1}-L_{1}^{2} .
\end{aligned}
$$

Assim, se $G$ é unimodular $L$ é simétrica e claramente vale a reciproca.

Lema 2.3.8 Se o grupo de Lie $G$ de dimensão 3 não é unimodular, então sua álgebra de Lie g possui uma base $\left\{e_{1}, e_{2}, e_{3}\right\}$ tal que

$$
\begin{aligned}
& {\left[e_{1}, e_{2}\right]=a e_{2}-b e_{3}} \\
& {\left[e_{1}, e_{3}\right]=d e_{1}+d e_{3}} \\
& {\left[e_{2}, e_{3}\right]=0}
\end{aligned}
$$


$e a+d=2$.

Demonstração: como $[\mathfrak{g}, \mathfrak{g}] \subset \mathcal{U}$, segue que $\operatorname{dim} \mathcal{U}=2$, caso contrário $G$ seria unimodular. Sendo $\mathcal{U}$ uma álgebra de Lie de dimensão 2 e unimodular, segue que $\mathcal{U}$ é comutativa. Podemos então tomar $e_{1} \in \mathfrak{g} \backslash \mathcal{U}$ tal que $\operatorname{tr}\left[\operatorname{ad}\left(e_{1}\right)\right]=2$ e portanto $T: \mathcal{U} \rightarrow \mathcal{U}$ dada por $T(X)=\left[e_{1}, X\right]$ possui traço $2(\operatorname{tr}(T)=2)$.

Do lema anterior, existe uma base tal que $\left\{e_{1} ; e_{2}, \xi\right\}$

$$
\begin{aligned}
{\left[e_{1}, e_{2}\right] } & =\Gamma_{2} e_{2}-2 \xi \\
{\left[e_{1}, \xi\right] } & =\Upsilon e_{2} \\
{\left[e_{2}, \xi\right] } & =0 .
\end{aligned}
$$

Vejamos qual é o grupo $G$ associado à álgebra $\mathfrak{g}$, para a qual existe uma base nas condições do lema 2.3.8. O subgrupo $H$, simplesmente conexo, obtido integrando $\mathcal{U}$ é claramente $\mathbb{R}^{2}\left(H=\mathbb{R}^{2}\right)$. Como $\left[e_{1}\right]$ é uma sub-álgebra, integrando-a obteremos $\mathbb{R}$. $a d\left(e_{1}\right)$ induz uma aplicação de $\mathbb{R}$ em $g l\left(\mathbb{R}^{2}\right)=g l(\mathcal{U})$, dada por $t \rightarrow a d\left(t e_{1}\right)$. Podemos então definir

$$
\sigma(t)=\exp \left(a d\left(t e_{1}\right)\right)
$$

Portanto, $G=\mathbb{R} \times \mathbb{R}^{2}$ com produto dado por

$$
(t,(x, y)) \cdot(s,(z, w))=\left(t+s, \exp \left(a d\left(t e_{1}\right)\right)(z, w)+(x, y)\right)
$$

Resta-nos então calcular $\exp \left(\operatorname{ad}\left(t e_{1}\right)\right)$.

Mas sendo $A=\left[\begin{array}{cc}\Gamma_{2} & \Upsilon \\ -2 & 0\end{array}\right]$ o calculo de $e^{t A}$ dependerá da relaçao entre $\Gamma_{2}$ e $\Upsilon$. De fato, calculando os auto-valores encontramos,

$$
x_{1}=\frac{\Gamma_{2}+\sqrt{\Gamma_{2}^{2}-8 \Upsilon}}{2} \text { e } \quad x_{2}=\frac{\Gamma_{2}-\sqrt{\Gamma_{2}^{2}-8 \Upsilon}}{2}
$$

Temos então dois casos: 
CASO I: $\quad \Gamma_{2} \neq 2 \sqrt{2 \Upsilon}$. Neste caso $A$ é diagonalizável e

$$
\begin{gathered}
{\left[\begin{array}{ll}
\Gamma_{2} & \Upsilon \\
-2 & 0
\end{array}\right]=\left[\begin{array}{cc}
-\Upsilon & -\Upsilon \\
x_{2} & x_{1}
\end{array}\right]\left[\begin{array}{cc}
x_{1} & 0 \\
0 & x_{2}
\end{array}\right]\left[\begin{array}{cc}
x_{1} & \Upsilon \\
-x_{2} & -\Upsilon
\end{array}\right] .} \\
\operatorname{expt}\left[\begin{array}{ll}
\Gamma_{2} & \Upsilon \\
-2 & 0
\end{array}\right]=\left[\begin{array}{cc}
-\Upsilon & -\Upsilon \\
x_{2} & x_{1}
\end{array}\right]\left[\begin{array}{cc}
e^{t x_{1}} & 0 \\
0 & e^{t x_{2}}
\end{array}\right]\left[\begin{array}{cc}
x_{1} & \Upsilon \\
x_{2} & -\Upsilon
\end{array}\right]
\end{gathered}
$$

. Para $\alpha=\sqrt{\Gamma_{2}^{2}-8 \Upsilon}$ teremos

$$
\exp (t A)=\left[\begin{array}{cc}
-\Gamma_{2} \sinh \frac{\alpha}{2} t+\alpha \cosh \alpha 2 t & -\Upsilon \sinh \frac{\alpha}{2} t \\
2 \sinh \frac{\alpha}{2} t & -\Gamma_{2} \sinh \frac{\alpha}{2} t-\alpha \cosh \alpha 2 t
\end{array}\right]
$$

CASO II: $\Gamma_{2}^{2}=8 \Upsilon$. Neste caso temos $x_{1}=x_{2}=\frac{\Gamma_{2}}{2} \mathrm{e}$

$$
\left[\begin{array}{cc}
\Gamma_{2} & \Upsilon \\
-2 & 0
\end{array}\right]=\left[\begin{array}{cc}
-\Upsilon & -\Upsilon \\
0 & \frac{\Gamma_{2}}{2}
\end{array}\right]\left[\begin{array}{cc}
\frac{\Gamma_{2}}{2} & 0 \\
\frac{\Gamma_{2}}{2} & \frac{\Gamma_{2}}{2}
\end{array}\right]\left[\begin{array}{cc}
\frac{\Gamma_{2}}{2} & \Upsilon \\
0 & -\Upsilon
\end{array}\right]
$$

Portanto, para a matriz neste caso

$$
\exp (t A)=e^{\Gamma_{2}}\left\{\left[\begin{array}{cc}
\frac{\Gamma_{2}}{2} & \Upsilon \\
-2 & -\frac{\Gamma_{2}}{2}
\end{array}\right]+I\right\}
$$

Resumindo então, obtivemos para $B=\left[\begin{array}{cc}\Gamma_{2} & \Upsilon \\ -2 & 0\end{array}\right]$

$$
e^{t A}= \begin{cases}e^{\frac{\alpha}{2}}\left[\frac{2}{\alpha} \operatorname{senh}\left(\frac{\alpha \mathrm{t}}{2}\right) \mathrm{B}+\cosh \left(\frac{\alpha \mathrm{t}}{2}\right) \mathrm{I}\right] & , \text { se } \alpha>0 \\ e^{\frac{a t}{2}}[B+I] & , \text { se } \alpha=0 \\ e^{\frac{\alpha}{2}}\left[2 \operatorname{senh}\left(-\mathrm{i} \frac{\alpha}{2} \mathrm{t}\right) \mathrm{B}+\cos \left(\frac{\alpha}{2} \mathrm{t}\right) \mathrm{I}\right] & , \text { se } \alpha \in i \mathbb{R}\end{cases}
$$

Voltando ao caso unimodular, já sabemos que existe uma base $\left\{e_{1}, e_{2}, e_{3}\right\}$ de $\mathfrak{g}$ tal que

$$
\begin{aligned}
& {\left[e_{1}, e_{2}\right]=\lambda_{3} e_{3},} \\
& {\left[e_{2}, e_{3}\right]=\lambda_{1} e_{1},} \\
& {\left[e_{3}, e_{1}\right]=\lambda_{2} e_{2} .}
\end{aligned}
$$


Claramente os $\lambda_{i}$ 's dependem da orientação dada a $\mathfrak{g}$, mas se mudarmos a orientação os auto-valores ficam multiplicados por $(-1)$. Se assumirmos que $\mathcal{D}$ é gerada por $\left\{e_{1}, e_{2}\right\}$, teremos $\xi \in\left[e_{3}\right]$ e tal que

$$
\begin{aligned}
{\left[e_{1}, e_{2}\right] } & =-2 \xi \\
{\left[e_{2}, \xi\right] } & =\lambda_{1} e_{1} \\
{\left[\xi, e_{1}\right] } & =\lambda_{2} e_{2}
\end{aligned}
$$

Temos então os seguintes casos:

(1) se $\lambda_{1}=l_{2} b=-1$, os grupos simplesmente conexos associados a esta álgebra são o $S^{3}$ e $S O(3)$;

(2) se $\lambda_{1}=-1$ e $\lambda_{2}=1$, os grupos de Lie simplesmente conexos associados são $S L(2, \mathbb{R})$ e $O(1,0)$;

(3) se $\lambda_{1}=-1$ e $\lambda_{2}=0$, o grupo associado é o $E(2)$;

(4) se $\lambda_{1}=1$ e $\lambda_{2}=0$, o grupo associado é o $E(1,1)$;

(5) se $\lambda_{1}=\lambda_{2}=0$, o grupo associado é o grupo de Heisenberg.

No caso unimodular, comparando com (1.1) temos que $\Gamma_{1}=\Gamma_{2}=0$. Se tomarmos uma métrica sobre $\mathcal{D}$ tal que $e_{1}, e_{2}$ são ortogonais unitários, teremos que: para $S^{3}, K=2$, $w_{1}=0$ e $w_{2}=0$; para $S L(2, \mathbb{R})$ temos $K=0, w_{1}=0$ e $w_{2}=0$; para $E(2)$ temos $K=1$, $w_{1}=0$ e $w_{2}=0$; para $E(1,1)$ temos $K=-1, w_{1}=0$ e $w_{2}=0$. Para o grupo de Heisenberg todas as curvaturas são nulas.

\section{O grupo de Heisenberg Generalizado}

Em $\mathbb{C}^{n+2}$ consideremos $b(z, w)=z_{1} \bar{w}_{1}+\cdots+z_{n} \bar{w}_{n}-i\left(z_{0} \bar{w}_{n+1}-z_{n+1} \bar{w}_{0}\right)$ e definamos

$$
Q^{n}=\left\{z=\left(1, z_{1}, \ldots, z_{n+1}\right) \in \mathbb{C}^{n+2} \mid b(z, z)=0\right\},
$$

isto é, se $z_{n+1}=x+i y$ para $x$ e $y$ reais,

$$
Q^{n}=\left\{\left(1, z_{1}, \ldots, z_{n}, x+\frac{i}{2} \sum_{j=1}^{n}\left|z_{j}\right|^{2} \mid z_{j} \in \mathbb{C}, j=1, \ldots, n\right\} .\right.
$$


Claramente $Q^{n}$ é uma variedade. Seja $U(n+1,1)$ o seguinte conjunto de aplicações lineares

$$
U(n+1,1)=\{T \in G L(n+2, \mathbb{C}): b(T u, T v)=b(u, v)\}
$$

Se $T$ é tal que $T\left(e_{0}\right)=e_{0}+\xi_{1} e_{1}+\cdots+\xi_{n+1} e_{n+1}$, então $T\left(\left[e_{1}, \ldots, e_{n+1}\right]\right) \subset\left[e_{1}, \ldots, e_{n+1}\right]$ e, portanto, estas aplicações são da forma

$$
T=\left[\begin{array}{ll}
1 & 0 \\
\xi & A
\end{array}\right], \quad \xi \in \mathbb{C}^{n+1} \quad \text { e } \quad A \in G L(n+1, \mathbb{C}) .
$$

Se, além disso, $b(T u, T v)=b(u, v)$, teremos que

$$
T=\left[\begin{array}{ccc}
1 & 0 & 0 \\
\frac{z}{\sqrt{2}} & A & 0 \\
x+\frac{i}{2}\|z\|^{2} & i \frac{\bar{z}^{t}}{\sqrt{2}} A & 1
\end{array}\right]
$$

Portanto, o grupo $G$ tal que $T\left(e_{0}\right)=e_{0}+v, v \in\left[e_{1}, \ldots, e_{n+1}\right]$ e $T\left(\left[e_{1}, \ldots, e_{n+1}\right]\right)$ é dado por

$$
G=\left\{\left[\begin{array}{ccc}
1 & 0 & 0 \\
\frac{z}{\sqrt{2}} & A & 0 \\
x+\frac{i}{2}\|z\|^{2} & \frac{\bar{z}^{t}}{\sqrt{2}} A & 1
\end{array}\right] \mid A \in U(n), z \in \mathbb{C}^{n} \quad \text { e } \quad x \in \mathbb{R}\right\}
$$

Temos então uma ação de $G$ em $Q^{n}$,

$$
(g, z) \rightarrow g(z)
$$

É fácil verificar que esta ação é $\mathcal{C}^{\infty}$ e transitiva. A isotropia de $e_{0}$ é dada por $G_{0}=\{g \in$ $\left.G \mid g \cdot e_{0}=e_{0}\right\}$ e portanto um cálculo simples nos mostra que

$$
\begin{aligned}
& G_{0}=\left\{\left[\begin{array}{lll}
1 & 0 & 0 \\
0 & A & 0 \\
0 & 0 & 1
\end{array}\right] \mid A \in U(n)\right\} \\
& Q^{n} \cong G / G_{0} \cong\left\{\left[\begin{array}{ccc}
1 & 0 & 0 \\
\frac{z}{\sqrt{2}} & I & 0 \\
x+\frac{i}{2}\|z\|^{2} & i \frac{\bar{z}^{t}}{\sqrt{2}} & 1
\end{array}\right] \mid z \in \mathbb{C}^{n}, x \in \mathbb{R}\right\} .
\end{aligned}
$$

Isto mostra que $Q^{n}$ é homogênea. $Q^{n}$ pode ser identificada com $\mathbb{C}^{n} \times \mathbb{R}$. De $b(z, z)=0$ tiramos que

$$
\begin{aligned}
& b(z, d z)+b(d z, z)=0 \\
& \operatorname{Reb}(z, d z)=0 \\
& \sum_{j=1}^{n} \bar{z}_{j} d z_{j}+\frac{i}{2}\left(d z_{n+1}\right)+\sum_{j=1}^{n} z_{j} d \bar{z}_{j}-\frac{i}{2} d \bar{z}_{n+1}=0 .
\end{aligned}
$$


Seja

$$
\mathcal{D}_{z}=\left\{\left(v_{1}, \ldots, v_{n+1}\right) \in \mathbb{C}^{n+1} \mid \Sigma \bar{z}_{j} v_{j}+\frac{i}{2} v_{n+1}=0\right\} .
$$

$\mathcal{D}_{z} \subset T_{z} Q^{n}$. D é não-integrável. De fato, $v=\frac{\partial}{\partial z_{n}}+2 i \bar{z}_{n} \frac{\partial}{\partial z_{n+1}} \in \mathcal{D}_{z}$ se $v=v_{1}+i v_{2}$.

$$
\begin{aligned}
v & =\frac{\partial}{\partial x_{n}}+2 y_{n} \frac{\partial}{\partial x_{n+1}}+2 x_{n} \frac{\partial}{\partial y_{n+1}}+i\left[-\frac{\partial}{\partial y_{n}}+2 x_{n} \frac{\partial}{\partial x_{n+1}}-2 y_{n} \frac{\partial}{\partial y_{n+1}}\right], \\
v_{1} & =\frac{\partial}{\partial x_{n}}+2\left(y_{n} \frac{\partial}{\partial x_{n+1}}+x_{n} \frac{\partial}{\partial y_{n+1}}\right), \\
v_{2} & =-\frac{\partial}{\partial y_{n}}+2\left(x_{n} \frac{\partial}{\partial x_{n+1}}-y_{n} \frac{\partial}{\partial y_{n+1}}\right), \\
{\left[v_{1}, v_{2}\right] } & =2 \frac{\partial}{\partial x_{n+1}}+2 \frac{\partial}{\partial x_{n+1}}=4 \frac{\partial}{\partial x_{n+1}} \notin \mathcal{D}_{z}
\end{aligned}
$$

- Também é verdadeiro que $b$ restrita à distribuição $\mathcal{D}$ é positiva definida.

\subsection{Relação entre as conexões Riemanniana e sub- Riemanniana}

Associado a variedade Riemanniana $(M,\langle\rangle$,$) temos a conexão de Levi-Civita, isto é, a$ única conexão em $M$ que satisfaz:

(i) $X\langle Y, Z\rangle=\left\langle D_{X} Y, Z\right\rangle+\left\langle Y, D_{X} Z\right\rangle$;

(ii) $D_{X} Y-D_{Y} X=[X, Y]$ ( Torção nula );

Esta afirmação é demonstrada em qualquer livro de geometria Riemanniana, em especial é demonstrado em [do Carmo]. Relembrando a definição $\tilde{h}$ e $h$ temos que $d \theta(X, Y)=$ $\langle\tilde{h}(X), Y\rangle$ e $h(X)=\tilde{h}(X)-\theta(\tilde{h}(X)) \xi$ para todo $X \in \mathscr{X}(M)$. Temos então o seguinte teorema

Teorema 2.4.1 Para campos $X \in \mathscr{X}(M)$ e $Y \in \mathcal{D}$ verifica-se

(i) $D_{X} Y=\nabla_{X} Y+\theta(X) h(Y)-\left\langle D_{X} \xi, Y\right\rangle \xi$;

(ii) $D_{X} \xi=h(X)+\tau(X)$. 
Demonstração: definamos, para $X \in \mathscr{X}(M)$

$$
\widetilde{D}_{X} Y=\nabla_{X} Y+\theta(X) h(Y)-\langle h(X)+\tau(X), Y\rangle \xi+\theta(Y)(h(X)+\tau(X)-\theta(X) h(\xi))
$$

que $\widetilde{D}$ é uma conexão é imediato. $\widetilde{D}$ é compatível com a métrica. De fato, para $Y, Z \in \mathcal{D}$

$$
\begin{aligned}
X\langle Y, Z\rangle & =\left\langle\nabla_{X} Y, Z\right\rangle+\left\langle Y, \nabla_{X} Z\right\rangle \\
& =\left\langle\tilde{D}_{X} Y, Z\right\rangle+\left\langle Y, \tilde{D}_{X} Z\right\rangle+\theta(X)(\langle h(Y), Z\rangle+\langle Y, h(Z)\rangle \\
& =\left\langle\tilde{D}_{X} Y, Z\right\rangle+\left\langle Y, \tilde{D}_{X} Z\right\rangle
\end{aligned}
$$

para $Y \in \mathcal{D}$ e $Z=\xi$

que $\tilde{D}$ possui torção nula poderemos verificar através de um cálculo simples. Primeiramente suponhamos que os campos $X$ e $Y$ pertencem a distribuição $\mathcal{D}$

$$
\begin{aligned}
\tilde{T}(X, Y) & =\tilde{D}_{X} Y-\tilde{D}_{Y} X-[X, Y] \\
& =\nabla_{X} Y-\left\langle D_{X} \xi, Y\right\rangle-\nabla_{Y} X-\left\langle D_{Y} \xi, X\right\rangle \\
& =\left(\nabla_{X} Y-\nabla_{Y} X-[X, Y]\right)+\left(\left\langle D_{Y} \xi, X\right\rangle-\left\langle D_{X} \xi, Y\right\rangle\right) \xi \\
& =d \theta(X, Y) \xi+\theta([X, Y]) \xi \\
& =(d \theta(X, Y)+\theta([X, Y]) \xi \\
& =0 \\
\tilde{T}(\xi, X) & =\tilde{D}_{\xi} X-\tilde{D}_{X} \xi-[\xi, X] \\
& =\nabla_{\xi} X+h(X)-\left\langle D_{\xi} \xi, X\right\rangle \xi-h(X)-\tau(X)-[\xi, X] \\
& =\left(\nabla_{\xi} X-[\xi, X]\right)-\tau(X)+\left\langle D_{\xi} \xi, X\right\rangle \xi \\
& =0
\end{aligned}
$$




\section{Imersões Isométricas sub-Riemannianas}

Como já sabemos que a esfera $S^{2 n+1}$ ( o hiperboloide $Q_{r}^{2 n+1}$ ) é um fibrado principal sobre $\mathbb{C P}^{n}\left(\mathbb{C H}^{n}\right)$ com fibra $S^{1}$. Sabemos também que $\pi: S_{r}^{2 n+1} \rightarrow \mathbb{C P}^{n}\left(\pi: Q_{r}^{2 n+1} \rightarrow \mathbb{C H}^{n}\right)$ nos dá uma submersão Riemanniana e portanto as propriedades geométricas de uma submersão podem ser aplicadas para o estudo de uma hipervariedade $M$ em uma forma espacial complexa, isto é, podemos "levantar" M para uma $\bar{M}$ em $S_{r}^{2 n+1}\left(Q_{r}^{2 n+1}\right)$ e estudar as propriedades de $\bar{M}$ e tentar inferir resultados para $\mathrm{M}$ através dos resultados adquiridos de $\bar{M}$. Para tal, recordemos um pouco de geometria diferencial para uma imersão em um espaco de formas e para uma submersão.

\subsection{Hipervariedades}

Estaremos interessados em imersões isométricas de variedades sub-Riemannianas $(M, \mathcal{D},\langle\rangle$, em formas espaciais complexas $N(c)$ (curvatura holomorfa constante $c$ ) de modo que $\mathcal{D}_{p}=T_{p} M \cap J\left(T_{p} M\right)$, sendo $J$ a estrutura complexa de $N(c)$. Queremos então que $\mathcal{D}$ seja invariante pela ação de $J$. Algumas abordagens deste problema tem sido feita de variadas maneiras: podemos citar o estudo de estruturas CR e variedades Sasakianas [22]. De modo mais rigoroso, dada uma variedade sub-Riemanniana $(M, \mathcal{D}, \xi,\langle\rangle$,$) de dimensão$ $2 n+1$ queremos imergi-la em $N(c)$ de dimensão $2(n+1)$

$$
f:(M, \mathcal{D}, \xi) \longrightarrow N(c),
$$

de forma que

$$
d f(\mathcal{D})=d f(T M) \cap J[d f(T M)]
$$


Para análise do caso acima mencionado vejamos primeiramente o caso Riemanniano geral. Seja $N$ uma variedade Riemanniana de dimensão $n+1(\operatorname{dim} N=n+1) \operatorname{com}$ métrica $\langle$,$\rangle .$ Seja também $f: M \rightarrow N$ uma imersão isométrica de uma variedade Riemanniana $M$ de dimensão n. Identificaremos $M$ com sua imagem $f(M)$. Para campos locais $X$ e $Y$ tangentes a $M$ e $\eta$ normal a $M$, se denotarmos por $\tilde{D}$ e D as derivadas covariantes de $N$ e $M$ respectivamente, teremos que

$$
\begin{aligned}
\tilde{D}_{X} Y & =D_{X} Y+b(X, Y) \eta \\
\tilde{D}_{X} \eta & =-B(X) .
\end{aligned}
$$

Sendo $b$ denominada segunda forma fundamental e $B$ o tensor de Weingarten. Em [3] prova-se que $b$ e $B$ são tensores de tipo $(0,2)$ e $(1,1)$, respectivamente. Em [3] também se prova que $b$ é simétrica e que $B$ é simétrico em relaçao a métrica Riemanniana.

Sejam $\tilde{R}$ e $R$ os tensores curvatura de $N$ e $M$ respectivamente. Para campos $X, Y$ e $Z$ tangentes a $M$ e $\eta$ normal a $M$ teremos que

$$
\begin{aligned}
\tilde{D}_{X} \tilde{D}_{Y} Z & =\tilde{D}_{X}\left\{D_{Y} Z+b(Y, Z) \eta\right\} \\
& =D_{X} D_{Y} Z+b\left(X, D_{Y} Z\right) \eta+X b(Y, Z) \eta+b(Y, Z) \tilde{D}_{X} \eta \\
& =D_{X} D_{Y} Z-b(Y, Z) B(X)-\left[X b(Y, Z)+b\left(Y, D_{X} Z\right)\right] \eta
\end{aligned}
$$

$$
\begin{aligned}
\tilde{D}_{Y} \tilde{D}_{X} Z & =\tilde{D}_{Y}\left\{D_{X} Z+b(X, Z) \eta\right\} \\
& =D_{Y} D_{X} Z+b\left(Y, D_{X} Z\right) \eta+Y b(X, Z) \eta+b(X, Z) \tilde{D}_{Y} \eta \\
& =D_{Y} D_{X} Z-b(X, Z) B(Y)-\left[Y b(X, Z)+b\left(Y, D_{X} Z\right)\right] \eta
\end{aligned}
$$

$$
\tilde{D}[X, Y] Z=D_{[X, Y]} Z+b([X, Y], Z) \eta
$$

Portanto, a relação entre $\tilde{R}$ e $R$ é dada por

$$
\tilde{R}(X, Y) Z=R(X, Y) Z+b(X, Z) B(Y)-b(Y, Z) B(X)+\langle\widetilde{R}(X, Y) Z, \eta\rangle \eta
$$


sendo

$$
\langle\widetilde{R}(X, Y) Z, \eta\rangle=X b(Y, Z)-Y b(X, Z)+b\left(X, D_{Y} Z\right)-b\left(Y, D_{X} Z\right)-b([X, Y], Z) .
$$

Para $W$ tangente a $M$ as equações acima nos dão as equações de Gauss

$$
\langle\tilde{R}(X, Y) Z, W\rangle=\langle R(X, Y) Z, W\rangle+b(X, Z) b(Y, W)-b(X, W) b(Y, Z),
$$

e olhando para a componente normal temos as equações de Mainardi-codazzi

$$
\begin{aligned}
\langle\tilde{R}(X, Y) Z, \eta\rangle & =X b(Y, Z)-Y b(X, Z)+b\left(X, D_{Y} Z\right)-b\left(Y, D_{X} Z\right)-b([X, Y], Z) \\
& =\left[X b(Y, Z)-b\left(D_{X} Y, Z\right)-b\left(Y, D_{X} Z\right)\right]-\left[Y b(X, Z)-b\left(D_{Y} X, Z\right)-b\left(X, D_{Y} Z\right)\right] \\
& =\left(D_{X} b\right)(Y, Z)-\left(D_{Y} b\right)(X, Z) \\
& =\left\langle\left(\left(D_{X} B\right)(Y)-\left(D_{Y} B\right)(X), Z\right\rangle\right.
\end{aligned}
$$

que serão bastante usadas em seç̧ões futuras. Algumas vezes no texto $R(X, Y) Z$ será denotado por $R(X \wedge Y) Z$. Sendo $\wedge$ usado somente para indicar anti-simetria em $X$ e $Y$.

\subsection{Submersões}

Sejam $M$ e $N$ variedades diferenciáveis de dimenões $n$ e $n+1$ respectivamente e $\pi$ : $N \rightarrow M$ uma submersão ( $d \pi$ sobrejetora ). Sabemos que para todo $x \in M, \pi^{-1}(x)$ é uma subvariedade diferenciável unidimensional de $N$, que costumeiramente é denominada fibra da submersão $\pi$ sobre $x$, ou brevemente, fibra sobre $x$. Aqui suporemos $\pi^{-1}(x)$ conexa para todo $x \in M$. Supondo $N$ uma variedade Riemanniana com um campo Killing $\xi$ tangente às fibras da submersão $\pi$ poderemos munir $M$ de uma métrica Riemanniana $\langle,\rangle_{M}$. Em verdade, é suficiente impor

$$
\left\langle d \pi\left(X_{y}\right), d \pi\left(Y_{y}\right)\right\rangle_{M}=\left\langle X_{y}, Y_{y}\right\rangle_{N}
$$

para $X_{y}, Y_{y} \in T_{y} N$. Como todo vetor tangente a $M$ é da forma $d \pi(V)$ para algum $V$ tangente a $N$ e $\xi$ é geradopor isometrias ao longo das fibras segue que acima temos uma boa definição. Em $N$ temos uma distribuição $\mathcal{H}$ dada por

$$
\mathcal{H}_{p}=\left\{u \in T_{p} N:\left\langle u, \xi_{p}\right\rangle=0\right\} \text {. }
$$

Um campo vetorial $Y$ será denominado horizontal se ele é $\pi$ relacionado com um campo $X$ de $M(d \pi(Y)=X \circ \pi)$ e $Y_{p} \in \mathcal{H}_{p}$ para todo ponto $p \in N$; neste caso $Y$ será denotado por $\widetilde{X}$ e será denominado levantamento de $X$. Um campo $X$ é vertical se $d \pi(X)=0$. 
Semelhantemente ao que foi feito para uma imersão, no caso de uma submersào $\pi$ também temos campos tensoriais associados, estes surgirão do lema abaixo.

Lema 3.2.1 Sejam $\widetilde{D}$ e $D$ as derivadas covariantes riemannianas de $N$ e $M$ respectivamente. Para campos $X$ e $Y$ tangentes a $M$ sejam $\widetilde{X}$ e $\widetilde{Y}$ os seus respectivos levantamentos, então

i) $\langle\tilde{X}, \widetilde{Y}\rangle_{N}=\langle X, Y\rangle_{M} \circ \pi$;

ii) $[\tilde{X}, \tilde{Y}]-\langle[\tilde{X}, \tilde{Y}], \xi\rangle_{N} \xi=\widetilde{[X, Y]}$;

iii) $\widetilde{D}_{\tilde{X}} \widetilde{Y}-\widetilde{D_{X} Y}$ é vertical;

iv) $D_{\tilde{X}} \xi=-\widetilde{A}(\widetilde{X})$ é horizontal.

Demonstração: Como $X=d \pi(\widetilde{X})$ e $Y=d \pi(\widetilde{Y})$ i) é imediato. A verificação de ii) também é direta

$$
d \pi\left([\tilde{X}, \tilde{Y}]-\langle[\widetilde{X}, \widetilde{Y}], \xi\rangle_{N} \xi\right)=d \pi([\tilde{X}, \widetilde{Y}])=[X, Y]
$$

Para provar iii) notemos que $\widetilde{X}\langle\widetilde{Y}, \widetilde{Z}\rangle=X\langle Y, Z\rangle \circ \pi$. Daí, usando a fórmula de Koszul para a conexão de Levi-Civita, teremos

$$
\begin{aligned}
2\left\langle\tilde{D}_{\tilde{X}} \tilde{Y}, \tilde{Z}\right\rangle & =\tilde{X}\langle\tilde{Y}, \tilde{Z}\rangle+\tilde{Y}\langle\tilde{X}, \tilde{Z}\rangle-\tilde{Z}\langle X, Y\rangle-\langle\tilde{X},[\tilde{Y}, \tilde{Z}]\rangle+\langle\tilde{Y},[\tilde{Z}, \tilde{X}]\rangle+\langle\widetilde{Z},[\tilde{X}, \tilde{Y}]\rangle \\
& =(X\langle Y, Z\rangle+Y\langle X, Z\rangle-Z\langle Y, X\rangle-\langle X,[Y, Z]\rangle+\langle Y,[Z, X]\rangle+\langle Z,[X, Y]\rangle) \circ \pi \\
& =2\left\langle D_{X} Y, Z\right\rangle \circ \pi,
\end{aligned}
$$

$\operatorname{logo} \widetilde{D}_{\tilde{X}} \tilde{Y}$ e $D_{X} Y$ são $\pi$-relacionados. (iv) é verificado pelo fato que $\xi$ é um campo de Killing unitário.

A componente vertical de $\widetilde{D}_{\tilde{X}} \widetilde{Y}$ denotaremos por $a(\tilde{X}, \tilde{Y})$. Verifica-se que $a(\tilde{X}, \tilde{Y})=$ $\langle\tilde{A}(X), \tilde{Y}\rangle_{N}$

Lema 3.2.2 Sejam a e A como no lema anterior, então

(i) $a(\tilde{X}, \tilde{X})=0$ para todo $X \in \mathscr{X}(M)$;

(ii) a é um tensor de tipo (0,2) sobre o anel das funções que são levantamentos; 
(iii) A é um tensor de tipo $(1,1)$.

Demonstração: De $\langle\widetilde{X}, \widetilde{X}\rangle$ ser constante ao longo das fibras teremos que

$$
\begin{aligned}
0 & =\xi\langle\tilde{X}, \tilde{X}\rangle \\
& =\left\langle D_{\xi} \tilde{X}, \widetilde{X}\right\rangle+\left\langle\widetilde{X}, D_{\xi} \widetilde{X}\right\rangle \\
& =2\left\langle D_{\xi} \widetilde{X}, \widetilde{X}\right\rangle \\
& =-2\left\langle\widetilde{D}_{\tilde{X}} \widetilde{X}, \xi\right\rangle \\
& =-2 a(\widetilde{X}, \tilde{X}) .
\end{aligned}
$$

Assim, (i) está demonstrado. Para a prova de (ii) tomemos $f \in \mathcal{C}^{\infty}(M)$ e seja $\tilde{f}=f \circ \pi$ teremos então

$$
\begin{aligned}
\widetilde{D}_{\tilde{f} \tilde{X}+\widetilde{Z}} \widetilde{Y} & =\widetilde{f} \widetilde{D}_{\widetilde{X}} \widetilde{Y}+D_{\widetilde{Z}} \widetilde{Y} \\
\widetilde{D_{f X+Z} Y}+a(\tilde{f} \widetilde{X}+\widetilde{Z}, \tilde{Y}) \xi & =\widetilde{f} \widetilde{D_{X} Y}+\widetilde{f} a(\tilde{X}, \tilde{Y}) \xi+\widetilde{D_{Z} Y}+a(\widetilde{Z}, \widetilde{Y}) \xi
\end{aligned}
$$

da igualdade acima tiramos que

$$
a(\tilde{f} \widetilde{X}+\widetilde{Z}, \tilde{Y})=\widetilde{f} a(\tilde{X}, Y)+, \tilde{f} a(\tilde{X}, \widetilde{Y})+a(\widetilde{Z}, Y)
$$

(iii) pode ser concluída de $(i i)$ pelo fato que $a(\tilde{X}, \tilde{Y})=\langle A(\tilde{X}), \tilde{Y}\rangle$.

Lema 3.2.3 Para campos $X$ e $Y$ sobre $M$ temos $a(\tilde{X}, \tilde{Y})=\frac{1}{2}\langle[\tilde{X}, \tilde{Y}], \xi\rangle$.

\section{Demonstração:}

$$
\begin{aligned}
{[\tilde{X}, \tilde{Y}] } & =\widetilde{D}_{\tilde{X}} \tilde{Y}-\widetilde{D}_{\tilde{Y}} \tilde{X} \\
& =\widehat{[X, Y]}+\{a(\tilde{X}, \tilde{Y})-a(\tilde{,}, \widetilde{X})\} \xi \\
& =\widetilde{[X, Y]}+2 a(\tilde{X}, \tilde{Y}) \xi
\end{aligned}
$$

portanto, $2 a(\tilde{X}, \tilde{Y})=\langle[\tilde{X}, \tilde{Y}], \xi\rangle$.

Do lema logo acima temos que $a$ está relacionado com a integrabilidade de $\mathcal{H}$. 
Teorema 3.2.4 Seja $\pi: N \rightarrow M$ uma submersão Riemanniana e $X$ e $Y$ campos vetoriais sobre $M$ com levantamentos horizontais $\tilde{X}$ e $\tilde{Y}$ respectivamente. Então as curvaturas seccionais $K$ de $M$ e $\widetilde{K}$ de $N$ satisfazem

$$
\left.K(X, Y)=\widetilde{K}(\widetilde{X}, \widetilde{Y})+\frac{3}{4}\langle[\widetilde{X}, \widetilde{Y})], \xi\right\rangle^{2} .
$$

Demonstração: Lembremo-nos que $\widetilde{D}_{\tilde{X}} \widetilde{Y}=\widetilde{D_{X} Y}+a(\widetilde{X}, \widetilde{Y}) \xi$. Portanto,

$$
\begin{aligned}
& \widetilde{D}_{\widetilde{X}} \widetilde{D}_{\tilde{Y}} \widetilde{Z}=\widetilde{D_{X} D_{Y} Z}+a(\widetilde{Y}, \widetilde{Z}) \widetilde{D}_{\tilde{X}} \xi+\left\{\widetilde{X} a(\tilde{Y}, \widetilde{Z})+a\left(\tilde{Y}, \widetilde{D_{Y} Z}\right\} \xi\right. \\
& \widetilde{D}_{\widetilde{Y}} \widetilde{D}_{\tilde{X}} \widetilde{Z}=\widetilde{D_{Y} D_{X} Z}+a(\tilde{X}, \widetilde{Z}) \widetilde{D}_{\widetilde{Y}} \xi+\left\{\widetilde{Y} a(\tilde{X}, \widetilde{Z})+a\left(\tilde{X}, \widetilde{D_{X} Z}\right\} \xi\right. \\
& \widetilde{D}_{[\tilde{X}, \tilde{Y}]} Z=\widetilde{D_{[X, Y]}}+\langle[\widetilde{X}, \widetilde{Y}], \xi\rangle \widetilde{D}_{\xi} \widetilde{Z}+a([\tilde{X}, \widetilde{Y}], \widetilde{Z}) \xi .
\end{aligned}
$$

Supondo $W \in \mathscr{X}(M)$ teremos

$\langle\widetilde{R}(\widetilde{X}, \widetilde{Y}) \widetilde{Z}, \widetilde{W}\rangle=\langle R(X, Y) Z, W\rangle+a(\widetilde{Y}, \widetilde{Z})\left\langle D_{\widetilde{X}} \xi, \widetilde{W}\right\rangle-a(\widetilde{X}, \widetilde{Z})\left\langle D_{\tilde{Y}} \xi, \widetilde{W}\right\rangle-\langle[\widetilde{X}, \widetilde{Y}], \xi\rangle\left\langle\widetilde{D_{\xi}} \widetilde{Z}, \widetilde{W}\right\rangle$

substituindo $Z=Y$ e $W=Y$, supondo-os unitários e usando que $a(\widetilde{X}, \widetilde{Y})=\left\langle\widetilde{D}_{\widetilde{X}} \widetilde{Y}, \xi\right\rangle=$ $-\left\langle\widetilde{D}_{\tilde{X}} \xi, Y\right\rangle$

$$
\begin{aligned}
\widetilde{K}(\tilde{X}, \widetilde{Y}) & =K(X, Y)+a(\tilde{X}, \widetilde{Y}) a(\tilde{Y}, \widetilde{X})+2 a(\tilde{X}, \tilde{Y}) a(\tilde{Y}, \tilde{X}) \\
& =K(X, Y)-3 a(\tilde{X}, \widetilde{Y})^{2}
\end{aligned}
$$

e isto encerra a demonstração.

O teorema acima nos permite, por exemplo, relacionar as curvaturas seccionais da esfera $S_{r}^{2 n+1}$ com as do $\mathbb{C I P}^{n}$. De fato, já sabemos que $\pi: S_{r}^{2 n+1} \longrightarrow \mathbb{C I P}^{n}\left(\frac{4}{r^{2}}\right)$, que associa a cada $z \in S_{r}^{2 n+1}$ a classe de equivalência [z] é uma submersão Riemanniana (ver capítulo 1). Que $\xi=i z$ é um campo de Killing é decorrente da expressão de seu fluxo $\varphi$ :

$$
\varphi_{t}(z)=e^{i t} z
$$

Resta-nos então descobrir a expressão que descreve $a(\tilde{X}, \tilde{Y})$, para levantamentos de campos $X$ e $Y$ unitários em $\mathscr{X}\left(\mathbb{C P}^{n}\right)$

$$
\begin{aligned}
a(\widetilde{X}, \widetilde{Y}) & =\left\langle\widetilde{D}_{\tilde{X}} \widetilde{Y}, \xi\right\rangle \\
& =\left\langle\widetilde{D}_{\widetilde{X}} J \widetilde{Y}, J \xi\right\rangle
\end{aligned}
$$




$$
\begin{aligned}
& =\left\langle\widetilde{D}_{\tilde{X}} J \xi, J \widetilde{Y}\right\rangle \\
& =\frac{1}{r}\left\langle\widetilde{D}_{\tilde{X}} z, J \widetilde{Y}\right\rangle \\
& =\frac{1}{r}\langle\widetilde{X}, J \widetilde{Y}\rangle .
\end{aligned}
$$

Portanto, as curvaturas seccionais se relacionam pela fórmula

$$
k(X, Y)=\frac{1}{r^{2}}+\frac{3}{r^{2}}\langle\widetilde{X}, J \widetilde{Y}\rangle^{2}
$$

Que, obviamente, coincide com o resultado já encontrado no primeiro capítulo.

Consideremos duas submersões $\pi_{j}: N_{j} \rightarrow M_{j}$, para $j=1,2$, com fibras unidimensionais conexas e duas imersões $f_{N}: N_{1} \rightarrow N_{2}$ e $f_{M}: M_{1} \rightarrow M_{2}$ tais que as variedades imersas possuem codimensão 1 e, além disso, o diagrama abaixo comuta e leva fibra em fibra difeomorficamente

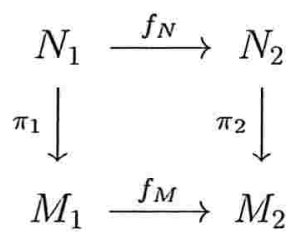

sob estas hipóteses, temos que $\xi_{N_{2}}=d f_{N}\left(\xi_{N_{1}}\right)$ e que $\widetilde{d f_{M}(X)}=d f_{N}(\widetilde{X})$. Teremos também que se $\eta$ é um campo normal unitário, em uma vizinhança de um ponto $x \in M_{1}$, então $\tilde{\eta}$ será um campo unitário normal a $N_{1}$.

Denotando por $\tilde{D}^{1}$ e $\tilde{D}^{2}$ as derivadas covariantes de $N_{1}$ e $N_{2}$ respectivamente e por $D^{1}$ e $D^{2}$ as derivadas covariantes de $M_{1}$ e $M_{2}$ respectivamente teremos que

$$
\begin{aligned}
& \tilde{D}_{d f_{N}(\tilde{X})}^{2} d f_{N}(\tilde{Y})=d f_{N}\left(\tilde{D}_{\tilde{X}}^{1} \tilde{Y}\right)+\langle B(\tilde{X}), \tilde{Y}\rangle \tilde{\eta} \\
&=d f_{N}\left(D_{\tilde{X}}^{\tilde{1}} \tilde{Y}+\langle\tilde{A}(\tilde{X}), \tilde{Y}\rangle \xi\right)+\langle B(\tilde{X}), \tilde{Y}\rangle \tilde{\eta} \\
&\left.=d f_{N}\left(D_{\tilde{X}}^{1} \tilde{Y}\right)+\langle\tilde{A}(\tilde{X}), \tilde{Y}\rangle \xi_{N_{2}}\right)+\langle B(\tilde{X}), \tilde{Y}\rangle \tilde{\eta} \\
& \tilde{D}_{d f_{N}(\tilde{X})} d f_{N}\left(\xi_{N_{1}}\right)=d f_{N}\left(\tilde{D}_{\tilde{X}}^{1} \xi_{N_{1}}\right)+\left\langle B\left(\xi_{N_{1}}\right), \tilde{X}\right\rangle \tilde{\eta} .
\end{aligned}
$$

É fácil verificar que

$$
\begin{array}{r}
A_{2} \circ d f_{M}(X)=d f_{N} \circ A_{1}(X)+\langle X, U\rangle \eta \\
A_{2}(\eta)=-d f_{M}(U)
\end{array}
$$


para um certo $\mathrm{U}$ tangente a $M_{1}$.

Lema 3.2.5 No diagrama acima se o tensor de Weigarten $B_{N}$ for paralelo, então $B_{M}$ e $A_{2}$ comutam $\left(B_{M} \circ A_{2}=A_{2} \circ B_{M}\right)$.

\section{Demonstração:}

$$
\begin{aligned}
0 & =\xi_{N_{2}}\left\langle B_{M} X, Y\right\rangle \circ \pi \\
& =\xi_{N_{2}}\left\langle\widetilde{B_{M} X}, \widetilde{Y}\right\rangle \\
& =\xi_{N_{2}}\left\langle B_{n}(\tilde{X}), \widetilde{Y}\right\rangle \\
& =\left\langle\left(D_{\xi_{N_{2}}} B_{N}\right)(\widetilde{X})+B_{N}\left(\widetilde{D}_{\xi_{N_{2}}} \widetilde{X}\right), \widetilde{Y}\right\rangle+\left\langle B_{N} \widetilde{X}, \widetilde{D_{\xi_{N_{2}}}} \widetilde{Y}\right\rangle \\
& =\left\langle B_{N}\left(\widetilde{D_{\xi_{N}}} \widetilde{X}\right), \widetilde{Y}\right\rangle+\left\langle B_{N} \widetilde{X}, \widetilde{D_{\tilde{Y}}} \xi_{N}\right\rangle \\
& =-\left\langle B_{N}\left(\widetilde{A_{2}(X)}\right), \widetilde{Y}\right\rangle-\left\langle B_{N} \widetilde{X}, \widetilde{A_{2}(Y)}\right\rangle \\
& =\left\langle B_{M} A_{2}(X), \widetilde{Y}\right\rangle+\left\langle A_{2} \widetilde{B_{M}(X)}, \widetilde{Y}\right\rangle \\
& =\left\langle\left(A_{2} \circ B_{M}-B_{M} \circ A_{2}\right)(X), Y\right\rangle \circ \pi
\end{aligned}
$$

sendo $\langle$,$\rangle não degenerada segue o lema.$

A partir de agora, no diagrama acima, iremos supor $N_{2}=S^{2 n+1}$ e $M_{2}=\mathbb{C P}^{n}$. Mas, com mudanças convenientes em algumas das igualdades que aparecem, tudo continua válido para os espaços de curvatura holomorfa constante. Para este caso existe um campo $\xi$ satisfazendo $J \xi=\eta$. Os próximos lemas e teoremas serão enunciados tendo em mente que o diagrama acima é satisfeito para as variedades que fazem parte do enunciado.

Lema 3.2.6 Se uma imersão isométrica $f$, de uma variedade Riemanniana $M$ de dimensão $2 n+1$ em um espaço projetivo complexo $\mathbb{C P}^{n+1}\left(\frac{4}{r^{2}}\right)$, é tal que o tensor de Weingarten $B$ satisfaz $J B=B J$ e

$$
B^{2}(X)-\alpha B(X)-X+\langle\xi, X\rangle \xi=0 \quad \text { para todo } X \in \mathscr{X}(M) \quad \text { e alguma função } \quad \alpha,
$$

então a função $\alpha$ é constante.

Demonstração: do que fizemos acima segue que $\operatorname{grad}(\omega(\xi))=\beta \xi$, sendo $\beta=\xi \omega(\xi)$. Portanto,

$$
D_{X}(\operatorname{grad}(\omega(\xi))=X \beta \xi+\xi(\omega(\xi)) J B(X) .
$$

Do fato $\left\langle D_{X}(\operatorname{grad}(\omega(\xi)), Y\rangle=\left\langle X, D_{Y}(\operatorname{grad}(\omega(\xi))\rangle\right.\right.$ tiramos

$$
\begin{aligned}
X \beta\langle\xi, Y\rangle+\beta\langle J B(X), Y\rangle & =Y \beta\langle\xi, X\rangle+\beta\langle J B(Y), X\rangle \\
Y \beta\langle\xi, X\rangle-X \beta\langle\xi, Y\rangle & =2 \beta\langle J B(X), Y\rangle,
\end{aligned}
$$


para $X$ e $Y$ em $\mathcal{D}$ a igualdade acima se transforma em

$$
\beta\langle J B(X), Y\rangle=0 \text {. }
$$

Se em um ponto $p \in M$ temos $\beta \neq 0$ seremos levados a $J_{p} B_{p}\left(X_{p}\right)=0$ e aí teriamos um absurdo (usando o fato que $B^{2}(X)-\alpha B(X)-X+\langle\xi, X\rangle \xi=0$ ).

Lema 3.2.7 Se o tensor de Weingarten $B$ de uma imersão isométrica $f$ de uma variedade Riemanniana $M$ de dimensão $2 n+1$ em um espaço $\mathbb{C P}^{n+1}\left(\frac{4}{r^{2}}\right)$, satisfaz $B J=J B$ e $B^{2}(X)-\alpha B(X)-X+\langle\xi, X\rangle \xi=0$ para todo $X \in \mathscr{X}(M)$ e uma constante $\alpha$, então o tensor de Weingarten $\widetilde{B}$ do levantamento de $f$ à esfera de raio $r$ satisfaz

$$
\widetilde{B}^{2}(\widetilde{X})-\alpha \widetilde{B}(\widetilde{X})-\tilde{X}=0 \quad \text { para todo } \tilde{X} \in \mathscr{X}(\widetilde{M}) \text {. }
$$

Demonstração: Voltando ao diagrama acima e fazendo $M_{1}=M$ e tomemos $X \in \mathscr{X}\left(M_{1}\right)$. Como todo $Y \in \mathscr{E}\left(M_{2}\right)$ pode ser escrito na forma $Y=\widetilde{Z}+\left\langle Y, \xi_{N}\right\rangle \xi_{N}$, sendo $Z \in \mathscr{X}\left(M_{1}\right)$ teremos então

$$
\left\langle B_{N}^{2}(\widetilde{X})-\alpha B_{N}(\widetilde{X})-\widetilde{X}, Y\right\rangle=\left\langle B_{N}^{2}(\widetilde{X})-\alpha B_{N}(\widetilde{X})-\widetilde{X}, \widetilde{Z}\right\rangle+\left\langle B_{N}^{2}(\widetilde{X})-\alpha B_{N}(\widetilde{X})-\widetilde{X}, \xi_{N}\right\rangle
$$

De $B_{M}(\xi)=\left\langle B_{M}(\xi), \xi\right\rangle \xi=\alpha \xi$ e da simetria de $B_{M}$ segue que $\left\langle B_{M}(X), \xi\right\rangle=\alpha\langle X, \xi\rangle$. Verifica-se também que $\left\langle B_{N}\left(\xi_{N}\right), \widetilde{X}\right\rangle=-\langle\xi, X\rangle \circ \pi$ e que isto nos leva a $\left\langle B_{N}\left(\widetilde{B_{M}(X)}\right), \xi_{N}\right\rangle=$ $-\alpha\langle\xi, X\rangle \circ \pi$ usando também que $\left\langle B_{N}(\widetilde{X}), \widetilde{Z}\right\rangle=\left\langle B_{M}(X), Z\right\rangle \circ \pi$ tem-se

$$
\begin{aligned}
B_{N}(\tilde{X}) & =\widehat{B_{M}(X)}+\left\langle B_{N}(\tilde{X}), \xi_{N}\right\rangle \xi_{N} \\
& =\widehat{B_{M}(X)}-(\langle X, \xi\rangle \circ \pi) \xi_{N} .
\end{aligned}
$$

Portanto,

$$
B_{N}^{2}(\tilde{X})=\widehat{B_{M}^{2}(X)}-(\langle X, \xi\rangle \circ \pi) \xi_{N}-(\langle X, \xi\rangle \circ \pi) B_{N}\left(\xi_{N}\right)
$$

consequentemente temos

$$
\left\langle B_{N}^{2}(\tilde{X})-\alpha B_{N}(\tilde{X})-\tilde{X}, Y\right\rangle=\left\langle B_{M}^{2}(X)-\alpha B_{M}(X)-X+\langle X, \xi\rangle \xi, Y\right\rangle=0 .
$$

Teorema 3.2.8 Seja $M_{2}$ uma hipersuperfície do espaço projetivo complexo $\mathbb{C P}^{n}$ satisfazendo as condiçôes do diagrama acima. Para que $J$ e $B_{M}$ comutem é necessário e suficiente que $B_{N}$ seja paralelo. 
Demonstração: Suponhamos que $J \circ B_{N}=B_{N} \circ J$. Sabemos então que existem constantes $\alpha$ e $k$

$$
B_{N}^{2}(\tilde{X})=\alpha B_{N}(\tilde{X})+k \tilde{X}
$$

como $\left(D_{\widetilde{X}} B_{N}\right)(\tilde{Y})=\left(D_{\widetilde{Y}} B_{N}\right)(\tilde{X})$ e o tensor de $M_{2}$ se relaciona com o da esfera pela expressão abaixo

$$
\left.R(\widetilde{X}, \widetilde{Y})=k\langle\widetilde{Y}, \widetilde{Z}\rangle \widetilde{X}-\langle\widetilde{X}, \widetilde{Z})\rangle \widetilde{Y}+\left\langle B_{N}(\widetilde{Y}), \widetilde{Z}\right)\right\rangle B_{N}(\widetilde{X})-\left\langle B_{N}(\widetilde{X}), \widetilde{Z}\right\rangle B_{N}(\widetilde{Y})
$$

será válida então a igualdade

$$
\begin{aligned}
& D_{\tilde{X}} D_{\tilde{Y}} B_{N}-D_{\tilde{Y}} D_{\tilde{X}} B_{N}-D_{[\tilde{X}, \tilde{Y}]} \widetilde{Z}=R(\tilde{X}, \tilde{Y}) B_{N}(\widetilde{Z})-B_{N}(R(\widetilde{X}, \widetilde{Y}) \widetilde{Z}) \\
& \left.\left.=k\left\{\left\langle\tilde{Y}, B_{N} \widetilde{Z}\right\rangle \tilde{X}-\left\langle\tilde{X}, B_{N} \tilde{Z}\right)\right\rangle \tilde{Y}\right\}+\left\langle B_{N}(\tilde{Y}), B_{N} \widetilde{Z}\right)\right\rangle B_{N}(\tilde{X})-\left\langle B_{N}(\tilde{X}), B_{N} \widetilde{Z}\right\rangle B_{N}(\widetilde{Y}) \\
& \left.-k\left\{\langle\widetilde{Y}, \widetilde{Z}\rangle B_{N}(\widetilde{X})-\langle\widetilde{X}, \widetilde{Z})\right\rangle B_{N}(\widetilde{Y})\right\}-\left\langle B_{N}(\widetilde{Y}), \widetilde{Z}\right\rangle B_{N}^{2}(\widetilde{X}) \\
& +\left\langle B_{N}(\widetilde{X}), \widetilde{Z}\right\rangle B_{N}^{2}(\widetilde{Y}) \text {. }
\end{aligned}
$$

Seja $\left\{e_{1}(p) ; \ldots ; e_{2 n+1}(p)\right\}$ uma base ortonormal de $T_{p} S_{r}^{2 n+1}$. Podemos então estendê-la a uma base ortonormal em uma vizinhança de $p$ de maneira que $D e_{j}=0$ para $j=$ $1,2, \ldots, 2 n+1 . \operatorname{Em}_{\tilde{X}} D_{\widetilde{Y}} B_{N}-D_{\widetilde{Y}} D_{\tilde{X}} B_{N}-D_{[\tilde{X}, \tilde{Y}]} \widetilde{Z}=0$ substituindo $Y=Z=e_{j}$ e usando novamente que $\left(D_{w t i l X} B_{N}\right)(\widetilde{Y})=\left(D_{\widetilde{Y}} B_{N}\right)(\widetilde{X})$ e também que $\left.\sum_{j=1}^{2 n+1} D \widetilde{X}\left(D_{e_{j}} B_{N}\right)\right) e_{j}=0$ teremos

$$
\left.\left.\sum_{j=1}^{2 n+1} D_{e_{j}}\left(D_{\widetilde{X}} B_{N}\right)\right) e_{j}=\sum_{j=1}^{2 n+1} D_{e_{j}}\left(D_{e_{j}} B_{N}\right)\right) \tilde{X}=0
$$

usando que $\operatorname{tr}\left(B_{N}^{2}\right)$ é constante e derivando covariantemente $\operatorname{tr}\left(B_{N}^{2}\right)$ obtém-se

$$
\left.\frac{1}{2} \tilde{Y} \widetilde{X}\left(\operatorname{tr}\left(B_{N}^{2}\right)\right)=\operatorname{tr}\left(D_{\widetilde{Y}} D_{\widetilde{X}} B_{N}\right)\right) B_{N}+\operatorname{tr}\left[\left(D_{\widetilde{Y}} B_{N}\right)\left(D_{\widetilde{X}} B_{N}\right)\right]=0
$$

$\operatorname{para} \tilde{Y}=\tilde{X}$

$$
\operatorname{tr}\left(D_{\widetilde{X}} B_{N}\right)^{2}=-\operatorname{tr}\left(D_{\widetilde{X}}\left(D_{\widetilde{X}} B_{N}\right) B_{N}\right)=-\sum_{j=1}^{2 n+1}\left\langle\left(D_{\widetilde{X}}\left(D_{\widetilde{X}} B_{N}\right) e_{j}, B_{N} e_{j}\right\rangle .\right.
$$

Assim temos

$$
\left\langle D B_{n}, D B_{N}\right\rangle=\sum_{j=1}^{2 n+1} \operatorname{tr}\left(D_{e_{j}} B_{N}\right)^{2}=-\sum_{j, k=1}^{2 n+1}\left\langle\left(D_{e_{j}}\left(D_{e_{j}} B_{N}\right) e_{j}, B_{N} e_{k}\right\rangle=0\right.
$$

e isto finaliza a demonstração. 
Faremos agora alguns exemplos de imersões em $N(c)$.

\section{Exemplos:}

Em $\mathbb{C}^{n+2}$ sejam $S_{R}^{2 n+3}=\left\{\left(z_{1}, \ldots, z_{n+2}\right) \in \mathbb{C}^{n+2}: \sum_{j=1}^{n+2}\left|z_{j}\right|^{2}=R^{2}\right\}$ e $\tilde{S}=\left\{\left(z_{1}, \ldots, z_{n+2}\right) \in S_{R}^{2 n+3}: \sum_{j=1}^{n+1}\left|z_{j}\right|^{2}=r^{2}\left|z_{n+2}\right|^{2}\right\}$ Claramente $\tilde{S}$ é uma hipervariedade de $S_{R}^{2 n+3}$. Também é fácil ver que a ação do $S^{1}$ sobre $S_{R}^{2 n+3}$ deixa invariante $\tilde{S}$. Portanto, a projeção canônica de $S_{R}^{2 n+3}$ em $\mathbb{C P}^{n+1}$ projeta $\tilde{S}$ em uma hipervariedade $S=\pi(\tilde{S})$ de $\mathbb{P}^{n+1}$. Usando as equações

$$
\begin{gathered}
\sum_{j=1}^{n+2}\left|z_{j}\right|^{2}=R^{2} \\
\sum_{j=1}^{n+1}\left|z_{j}\right|^{2}=r^{2}\left|z_{n+2}\right|^{2}
\end{gathered}
$$

tiramos que

$$
S=\left\{[z] \in \mathbb{C P}^{n+1}: z_{1} \overline{z_{1}}+\ldots+z_{n+1} \bar{z}_{n+1}^{-}=\frac{(r R)^{2}}{1+r^{2}}, \quad\left|z_{n+2}\right|=\frac{R^{2}}{1+r^{2}}\right\}
$$

e daí $S$ é difeomorfa a uma esfera. Este exemplo é facilmente generalizado. De fato, em $S_{R}^{2 n+3}$ definamos

$$
\tilde{S}(n, m, r)=\left\{\left(z_{1}, \ldots, z_{n+2}\right) \in \mathbb{C}^{n+2}: \sum_{j=1}^{m}\left|z_{j}\right|^{2}=r^{2}\left(\sum_{j=m+1}^{n+2}\left|z_{j}\right|^{2}\right), \quad \sum_{j=1}^{n+2}\left|z_{j}\right|^{2}=R^{2}\right\} .
$$

Como $\tilde{S}(n, m, r)$ é invariante pela ação de $S^{1}$ em $S_{R}^{2 n+3}$ segue que sua projeção $S(m, n, r)=\pi[\tilde{S}(m, n, r)]$ é uma hipervariedade de $\mathbb{C P}^{n+1}$.

Do teorema 3.2 .8 e dos teoremas em [11] que caracterizam as hipervariedades homogêmas da esfera de raio $r$ segue que as variedades $S(m, n, r)$ do exemplo acima são as únicas hipersuperfícies homogêneas do espaço projetivo complexo ( $\mathbb{C P}^{n+1}\left(\frac{4}{r^{2}}\right)$ ) nas quais o tensor de Weingarten $B$ comuta com a estrutura complexa $J$.

\subsection{O caso sub-Riemanniano}

O caso de uma imersão isométrica $f$ de uma variedade sub-Riemanniana $(M, \mathcal{D}, \xi,\langle$,$\rangle em$ um espaço de forma complexa induzirá em $\mathcal{D}$ uma estrutura quase-complexa $J$ e $\eta=J(\xi)$ 
e podemos escrever para campos $X, Y \in \mathscr{E}(M)$

$$
\begin{aligned}
\tilde{D}_{X} Y & =D_{X} Y+b(X, Y) J \xi \\
\tilde{D}_{X} \eta & =-A(X)-\omega(X) \xi, \quad A(X) \in \mathcal{D} .
\end{aligned}
$$

De $b(X, Y)=\left\langle-\tilde{D}_{X} \eta, Y\right\rangle$ obtemos que

$$
\begin{array}{ll} 
& \left\langle\tilde{D}_{X} Y, \eta\right\rangle+\left\langle Y, \tilde{D}_{X} \eta\right\rangle=0, \\
& b(X, Y)=\left\langle-D_{X} \eta, Y\right\rangle=\langle A(X), Y\rangle+\omega(X)\langle Y, \xi\rangle \\
\mathrm{e} \quad & \omega(X)=b(X, \xi) .
\end{array}
$$

Proposição 3.3.1 A e w são tensores de tipo $(1,1)$ e $(0,1)$, respectivamente.

Demonstração: É imediato. De fato, de $\omega(X)=b(X, \xi)$ segue que $\omega$ é do tipo $(0,1)$ e do fato que $A(X)=-\tilde{D}_{X} \eta-\omega(X) \xi$ segue que $A$ é um tensor do tipo $(1,1)$.

Também podemos concluir que o operador linear que para todo $X \in \mathcal{D}$ associa $A(X)-\langle X, \xi\rangle \xi$ é simétrico. Como $\tilde{D} J=0$,

$$
\begin{aligned}
& \tilde{D}_{X} J Y=J \tilde{D}_{X} Y \\
& \nabla_{X} J Y+\theta(X) h \circ J(Y)+\left\langle D_{X} Y, \xi\right\rangle \xi+b(X, J Y) \eta \\
& =J \nabla_{X} Y+\theta(X) J \circ h(Y)-b(X, Y) \xi+\left\langle D_{X} Y, \xi\right\rangle \eta
\end{aligned}
$$

Desta equação tiramos que

$$
\begin{aligned}
& b(X, Y)=-\left\langle D_{X} J Y, \xi\right\rangle \quad \text { e } b(X, J Y)=\left\langle D_{X} Y, \xi\right\rangle, \\
& \tilde{D}_{X} Y=\nabla_{X} Y+\theta(X) h(Y)+b(X, J Y) \xi+b(X, Y) \eta
\end{aligned}
$$

(3.1) também nos mostra que

$$
\nabla_{X} J Y+\theta(X) h \circ J(Y)=J \nabla_{X} Y+\theta(X) J h(Y) .
$$

Para $X, Y \in \mathcal{D}$ teremos que

$$
\begin{aligned}
& \langle A \circ J(X), Y\rangle=b(J X, Y)=\left\langle D_{Y} X, \xi\right\rangle, \\
& \langle J \circ A(X), Y\rangle=-\langle A(X), J Y\rangle=-b(X, J Y)=\left\langle-D_{X} Y, \xi\right\rangle \\
& \langle(A \circ J+J \circ A)(X), Y\rangle=\langle[X, Y], \xi\rangle .
\end{aligned}
$$


Logo, se $\mathcal{D}$ é integrável, $A \circ J=-J \circ A$. Assim, $M$ é folheada e as folhas são hipervariedades complexas de $\tilde{N}$ e como $B \circ J=-J \circ B$, elas são mínimas. Em verdade, se $M_{1}$ é uma variedade integral de $\mathcal{D}$, teremos que o tensor de Weingarten será dado por $A$. Como $A$ é simétrico, existe uma base de auto-vetores para $A$. Se $e_{j}$ é um auto-vetor associado ao auto-valor $\lambda_{j}$, teremos que $A J e_{j}=-J A e_{j}=-J\left(\lambda_{j} e_{j}\right)=-\lambda_{j}\left(J e_{j}\right)$, portanto existe uma base de auto-vetores da forma $\mathcal{B}=\left\{e_{1}, \ldots, e_{n}, J e_{1}, \ldots, J e_{n}\right\}$, isto é, a matriz de $A$ em relação à base $\mathcal{B}$ é dada por

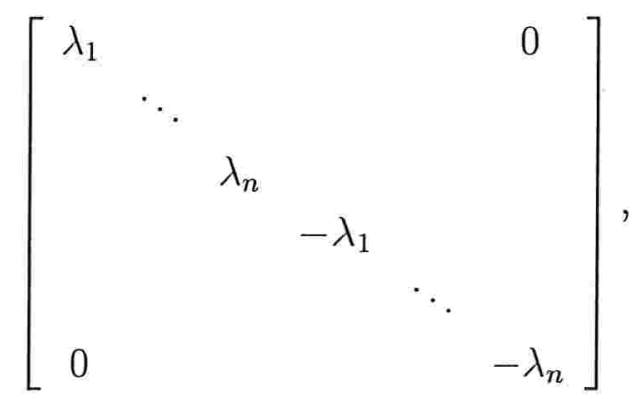

$\log 0, \operatorname{tr} A=0$.

Proposição 3.3.2 Seja $f: M \rightarrow N($ c) uma imersão isométrica de variedade sub-Riemanniana $(M, \mathcal{D}, g, \xi)$ de dimensão $2 n+1$ em um espaço de formas complexas $N(c)$ de dimensão complexa $n+1$. Suponhamos que $f$ é tal que $f_{*}(\mathcal{D})=f_{*}(T M) \cap J f_{*}(T M)$. Então a segunda forma fundamental fica determinada por

$$
b(X, Y)=-\left\langle J D_{X} \xi, Y\right\rangle-\left\langle J D_{\xi} \xi, X\right\rangle \theta(Y)+\omega(\xi) \theta(X) \theta(Y)
$$

e o tensor de Weingarten por

$$
B(X)=-J D_{X} \xi-\left[\left\langle D_{\xi} \xi, X\right\rangle-\omega(\xi) \theta(X)\right] \xi .
$$

\section{Demonstração:}

$$
\begin{aligned}
\tilde{D}_{X} \eta & =J D_{X} \xi-b(X, \xi) \xi \\
\omega(X) & =b(X, \xi)=\left\langle-\tilde{D}_{X} \eta, \xi\right\rangle=\left\langle-\tilde{D}_{\xi} \eta, X\right\rangle \\
& =\left\langle-J D_{\xi} \xi, X\right\rangle+\langle-b(\xi, \xi)(-\xi), X\rangle \\
& =-\left\langle J D_{\xi} \xi, X\right\rangle+\omega(\xi) \theta(X) \\
\tilde{D}_{X} \eta & =J D_{X} \xi+\left(\left\langle J D_{\xi} \xi, X\right\rangle-\omega(\xi) \theta(X)\right) \xi .
\end{aligned}
$$


Corolário 3.3.3 Nas condições da proposição logo acima, se as trajetórias de $\xi$ são geodésicas Riemannianas de $M$, então $\xi$ é uma curvatura principal de $M$

Demonstração: Se $D_{\xi} \xi=0$ então $B(\xi)$ será dado por

$$
\begin{aligned}
B(\xi) & =-J D_{\xi} \xi+\left[\left\langle D_{\xi} \xi, \xi\right\rangle-\omega(\xi) \theta(\xi)\right] \xi \\
& =-\omega(\xi) \xi .
\end{aligned}
$$

Da integrabilidade da estrutura complexa do espaço de forma complexa $N(c)$ temos que

$$
[J X, J Y]-[X, Y]=J([X, J Y])+J([J X, Y]) .
$$

Portanto, como $\mathcal{D}$ é invariante por $J$ teremos para $X$ e $Y$ em $\mathcal{D}$

$$
[J X, J Y]-[X, Y] \in \mathcal{D}
$$

e daí podemos escrever

$$
\begin{aligned}
& \theta([J X, J Y]-[X, Y])=0, \\
& \theta([J X, J Y])=\theta([X, Y]) .
\end{aligned}
$$

Lembremos que sendo $D$ a derivada covariante Riemanniana de $M$ temos $D \xi=\tau+h$.

Proposição 3.3.4 Nas condições acima teremos $\left.h\right|_{\mathcal{D}}$ holomorfo e $\left.\tau\right|_{\mathcal{D}}$ anti-holomorfo ( $h \circ J=J \circ h$ e $\tau \circ J=-J \circ \tau$ ).

Demonstração: Sabemos que $\theta([J X, J Y])=\theta(X, Y)$ para $X, Y \in \mathcal{D}$ e que

$$
d \theta(X, Y)=X \theta(Y)-Y \theta(X)-\theta([X, Y]) .
$$

Tomando $X, Y \in \mathcal{D}$,

$$
\begin{aligned}
& d \theta(X, Y)=-\theta([X, Y]), \\
& d \theta(J X, J Y)=-\theta([J X, J Y])=-\theta([X, Y])=d \theta(X, Y), \\
& \langle h \circ J X, J Y\rangle=\langle h(X), Y\rangle, \\
& \langle h \circ J X, J Y\rangle=\langle h(X), Y\rangle, \\
& \langle(J h-h \circ J)(X), Y\rangle=0
\end{aligned}
$$


e portanto $J \circ h=h \circ J$. Quanto a $\tau$ temos que $b(X, Y)=b(Y, X)$,

$$
\begin{aligned}
& \left\langle J D_{X} \xi, Y\right\rangle=\left\langle J D_{Y} \xi, X\right\rangle, \\
& \langle J h(X)+J \tau(X), Y\rangle=\langle J h(Y)+J \tau(Y), X\rangle, \\
& \langle(J h-h J)(X), Y\rangle=-\langle(J \tau+\tau J)(X), Y\rangle
\end{aligned}
$$

e portanto $J \circ \tau=-\tau \circ J$

Já vimos a relação entre $\tilde{R}$ e $R$ para uma hipervariedade isometricamente imersa, mas no caso sub-Riemanniano temos direções privilegiadas, a saber: $\mathcal{D}$ e $\xi$. Podemos para campos $X$ pertencente a $\mathscr{X}(M)$ e $Y$ pertencentes a $\mathcal{D}$ decompor $D_{X} Y$ em uma componente pertencente a $\mathcal{D}$, que denotaremos por $D_{X}^{T} Y$, e em sua componente na direção de $\xi$, que denotaremos por $k(X, Y)$ :

$$
D_{X} Y=D_{X}^{T} Y+k(X, Y) \xi
$$

De forma semelhante a feita para a segunda forma fundamental de uma imersão e para a derivada covariante induzida pelo espaço ambiente teremos que $k$ será um campo tensorial de tipo $(0,2)$ e $D^{T}$ será uma derivação para campos em $D$.

Proposição 3.3.5 Sejam $X$ e $Y$ campos em D. Então $D_{X}^{T} J Y=J D_{X}^{T} Y$ e $k(X, Y)=$ $b(X, J Y)$.

Demonstração: para campos $X \in \mathscr{X}(M)$ e $Y \in \mathcal{D}$ ( estamos identificando $\mathcal{D}$ com $d f(\mathcal{D})$ ) temos

$$
\begin{aligned}
J \widetilde{D}_{X} Y & =\widetilde{D}_{X} J Y \\
J\left(D_{X} Y+b(X, Y) J \xi\right) & =D_{X} J Y+b(X, J Y) J \xi \\
J\left(D_{X}^{T} Y+k(X, Y) \xi+b(X, Y) J \xi\right) & =D_{X}^{T} J Y+k(X, J Y) \xi+b(X, J Y) J \xi \\
J D_{X}^{T} Y-b(X, Y) \xi+k(X, Y) J \xi & =D_{X}^{T} J Y+k(X, J Y) \xi+b(X, J Y) J \xi
\end{aligned}
$$

da igualdade acima segue a proposição.

Definindo $R^{T}(X, Y) Z=D_{X}^{T} D_{Y}^{T} Z-D_{Y}^{T} D_{X}^{T} Z-D_{[X, Y]}^{T} Z$ obteremos a seguinte relação entre $R$ e $R^{T}$

$$
D_{X} D_{Y} Z=D_{X}\left\{D_{Y}^{T} Z+k(Y, Z) \xi\right\}
$$




$$
\begin{aligned}
& =D_{X} D_{Y}^{T} Z+k\left(X, D_{Y}^{T} Z\right) \xi+X k(Y, Z) D_{X} \xi \\
& =D_{X}^{T} D_{Y}^{T} Z+k(Y, Z) D_{X} \xi+\left(k\left(X, D_{Y}^{T} Z\right)+X k(Y, Z)\right) \xi
\end{aligned}
$$

$$
\begin{aligned}
D_{Y} D_{X} Z & =D_{Y}\left\{D_{X}^{T} Z+k(X, Z) \xi\right\} \\
& =D_{Y} D_{X}^{T} Z+k\left(Y, D_{X}^{T} Z\right) \xi+Y k(X, Z) D_{X} \xi \\
& =D_{Y}^{T} D_{X}^{T} Z+k(X, Z) D_{Y} \xi+\left(k\left(Y, D_{X}^{T} Z\right)+Y k(X, Z)\right) \xi
\end{aligned}
$$

$$
D_{[X, Y]}^{Z}=D_{[X, Y]}^{T} Z+k([X, Y], Z) \xi
$$

$$
R(X \wedge Y) Z=R^{T}(X \wedge Y) Z+k(Y, Z) D_{X} \xi-k(X, Z) D_{Y} \xi+\langle R(X \wedge Y) Z, \xi\rangle \xi
$$

Portanto, tiramos que

$$
\begin{aligned}
& \langle R(X \wedge Y) Z, \xi\rangle=X k(Y, Z)-Y k(X, Z)+k\left(X, D_{Y}^{T} Z\right)-k\left(Y, D_{X}^{T} Z\right)-k([X, Y], Z) \\
& \langle R(X \wedge Y) Z, \xi\rangle=X k(Y, Z)-Y k(X, Z)+k\left(X, D_{Y}^{T} Z\right)-k\left(Y, D_{X}^{T} Z\right)-k([X, Y], Z) .
\end{aligned}
$$

Substituindo $R(X, Y) Z$ nas equações de Gauss obteremos

$$
\begin{aligned}
\langle\tilde{R}(X, Y) Z, W\rangle= & \left\langle R^{T}(X \wedge Y) Z, W\right\rangle+k(Y, Z)\left\langle D_{X} \xi, W\right\rangle-k(X, Z)\left\langle D_{Y} \xi, W\right\rangle \\
& +\langle R(X \wedge Y) Z, \xi\rangle\langle W, \xi\rangle+b(X, Z) b(Y, W)-b(X, W) b(Y, Z),
\end{aligned}
$$

que para $W \in \mathcal{D}$ nos dará

$$
\begin{aligned}
\langle\tilde{R}(X, Y) Z, W\rangle= & \left\langle R^{T}(X \wedge Y) Z, W\right\rangle+k(Y, Z)\left\langle D_{X} \xi, W\right\rangle-k(X, Z)\left\langle D_{Y} \xi, W\right\rangle \\
& +b(X, Z) b(Y, W)-b(X, W) b(Y, Z)
\end{aligned}
$$

e para $W=\xi$ temos

$$
\langle\widetilde{R}(X, Y) Z, \xi\rangle=\langle R(X \wedge Y) Z, \xi\rangle+b(X, Z) b(Y, \xi)-b(X, \xi) b(Y, Z)
$$

que será usado na demonstração da proposição a seguir. 
Proposição 3.3.6 Dada uma imersão isométrica Riemanniana, $f$, de $(M, \mathcal{D}, \xi)$ em $N(c)$, se $d f(\mathcal{D})=d f(T M) \cap J d f(T M)$, então a curvatura de Ricci na direção $\xi(\operatorname{Ric}(\xi))$ é dada por

$$
\operatorname{Ric}(\xi)=n c+\omega(\xi) \operatorname{tr}(J \circ h)-\|J h(\xi)\|^{2}
$$

Demonstração: das equações de Gauss temos

$$
\langle\widetilde{R}(X, Y) Z, \xi\rangle=\langle R(X \wedge Y) Z, \xi\rangle+b(X, Z) b(Y, \xi)-b(X, \xi) b(Y, Z)
$$

Tomando uma base $\left\{e_{1} ; \ldots, e_{2 n}\right\}$ de $\mathcal{D}$

$$
\begin{aligned}
\operatorname{Ric}(\xi) & =\sum_{j=1}^{2 n}\left\langle R\left(\xi, e_{j}\right) e_{j}, \xi\right\rangle \\
& =\sum_{j=1}^{2 n}\left\{\left\langle\widetilde{R}\left(\xi, e_{j}\right) e_{j}, \xi\right\rangle+b(\xi, \xi) b\left(e_{j}, e_{j}\right)-b\left(\xi, e_{j}\right)^{2}\right\} \\
& =n c+\omega(\xi) \operatorname{tr}(B)-\|B(\xi)\|^{2}
\end{aligned}
$$

e suficiente notar que $B=J \circ(h+\tau)$, que $\tau$ é anti-holomorfo e que $\tau(\xi)=0$.

Definição 3.3.7 Denominaremos imersão sub-Riemanniana isométrica de $(M, \mathcal{D}, \xi, g)$ em uma forma espacial complexa $N(c)$ a toda imersão isométrica Riemanniana de $(M, \tilde{g})$ em $N(c)$ tal que $d f(\mathcal{D})=d f(T M) \cap J d f(T M)$.

Lema 3.3.8 Dadas duas imersões sub-Riemannianas isométricas $f$ e $\hat{f}$ em um espaço de forma complexa $N(c)$, se os tensores quase-complexos $J$ e $\hat{J}$ induzidos em $\mathcal{D}$ por $f$ e $\hat{f}$ respectivamente forem iguais, então as segundas formas $b$ de $f$ e $\hat{b}$ de $\hat{f}$ serão iguais.

Demonstração: É suficiente notar que para campos $X$ e $Y$ em $\mathscr{E}(M)$

$$
b(X, Y)=-\left\langle J D_{X} \xi, Y\right\rangle-\left\langle J D_{\xi} \xi,, X\right\rangle \theta(Y)-\omega(\xi) \theta(X) \theta(Y)
$$

e

$$
\hat{b}(X, Y)=-\left\langle\hat{J} D_{X} \xi, Y\right\rangle-\left\langle\hat{J} D_{\xi} \xi,, X\right\rangle \theta(Y)-\omega(\xi) \theta(X) \theta(Y)
$$


Teorema 3.3.9 Sejam $M$ uma variedade sub-Riemanniana de dimensão $2 n+1$ e $N(c)$ um espaço de forma complexa de dimensão complexa $n+1$. Dadas duas imersões isométricas sub-Riemannianas $f$ e $\hat{f}$ de $M$ em $N(c)$, então elas possuem uma direção principal em comum.

Demonstração: Denotaremos por $B$ e $\widehat{B}$ os tensores de Weingarten de $f$ e $\hat{f}$ respectivamente. Como $\left.\widetilde{B}\right|_{\mathcal{D}}$ é simétrico, localmente existe uma base ortonormal $\left\{e_{1} ; \ldots, 2_{2 n}\right\}$ de $\mathcal{D}$ que diagonaliza $\widetilde{B}$, isto é, uma base de autovetores. Sendo $\lambda_{j}$ o auto-valor associado a $e_{j}$ poderemos escrever

$$
\begin{aligned}
\widehat{B}\left(e_{k}\right) & =\lambda_{k} e_{k}+\hat{b}_{k} \xi \\
\widehat{B}(\xi) & =\sum_{k=1}^{2 n} \hat{b}^{k} e_{k}+\hat{\alpha} \xi \\
B\left(e_{k}\right) & =\sum_{j=1}^{2 n} b_{k}^{j} e_{k}+b_{k} \xi \\
B(\xi) & =\sum_{k=1}^{2 n} b^{k} e_{k}+\alpha \xi
\end{aligned}
$$

Olhando para as equações de Gauss teremos:

(i) usando $\left\langle\widetilde{R}\left(e_{j}, e_{k}\right) e_{l}, \xi\right\rangle=0$ obtemos para $j \neq k \neq l \neq j$

$$
b_{j}^{l} b_{k}-b_{k}^{l} b_{j}=0
$$

(ii) usando que $\left\langle\widetilde{R}\left(e_{j}, e_{k}\right) e_{k}, \xi\right\rangle=0$ para $j \neq k$ obtemos

$$
b_{k}^{j} b_{k}-b_{k}^{k} b_{j}=-\hat{b}_{j} \lambda_{k} ;
$$

(iii) usando que $\left\langle\widetilde{R}\left(e_{j}, \xi\right) e_{k}, \xi\right\rangle=0$ para $j \neq k$ obtemos

$$
b_{j}^{l} b_{k}-b_{k}^{l} b_{j}=0 \quad(j \neq k \neq l \neq j) ;
$$

(iv) façamos $e_{2 n+1}=\xi$ temos então

$$
\begin{aligned}
& \left\langle\widetilde{R}\left(e_{j}, e_{k}\right) e_{k}, e_{j}\right\rangle+b\left(e_{j}, e_{k}\right) b\left(e_{j}, e_{k}\right)-b\left(e_{j}, e_{j}\right) b\left(e_{k}, e_{k}\right)= \\
& \left\langle\widetilde{R}\left(e_{j}, e_{k}\right) e_{k}, e_{j}\right\rangle+\hat{b}\left(e_{j}, e_{k}\right) \hat{b}\left(e_{j}, e_{k}\right)-\hat{b}\left(e_{j}, e_{j}\right) \hat{b}\left(e_{k}, e_{k}\right) .
\end{aligned}
$$


Somando os dois lados de 1 até $2 n+1$ obtemos

$$
\left(b_{j}\right)^{2}+\sum_{k=1}^{2 n}\left(b_{j}^{k}\right)^{2}-b_{j}^{j}\left(\alpha+\sum_{k=1}^{2 n} b_{k}^{k}\right)=\left(\hat{b}_{j}\right)^{2}+\lambda_{j}^{2}-\lambda_{j}\left(\hat{\alpha}+\sum_{k=1}^{2 n} \lambda_{k}\right)
$$

(v) de $D_{X} Y=D_{X}^{T} Y+k(X, Y) \xi$ tiramos que $b(X, J Y)=\hat{b}(X, \widehat{J} Y)$ para todos os campos $X$ e $Y$ em $\mathcal{D}$. Portanto, tomando $j$ e $k$ em $\{1, \ldots ; 2 n\}$ a igualdade abaixo é satisfeita

$$
\begin{aligned}
\sum_{l=1}^{2 n} J_{l}^{j} b_{k}^{l} & =\sum_{l=1}^{2 n} \hat{J}_{l}^{j} \hat{b}_{k}^{l} \\
\sum_{l=1}^{2 n} J_{l}^{j} b_{k}^{l} & =\hat{J}_{k}^{j} \hat{\lambda}_{k} .
\end{aligned}
$$

Seja $r$ o número de $b_{j}$ não nulos. Temos então vários casos, façamos a demonstração dividindo-a em casos:

CASO I: $r=0$. Se $r=0$ temos $B(\mathcal{D}) \subset \mathcal{D}$. Como $B$ é simétrico $\xi$ é uma direção principal de $B$. Revisitando $(v)$

$$
\begin{aligned}
b(X, J(Y)) & =\hat{b}(X, \hat{J}(Y)) \\
\langle B(X), J(Y)\rangle & =\langle\hat{B}(X), \hat{J}(Y)\rangle
\end{aligned}
$$

façamos $X=\xi$

$$
\begin{aligned}
\langle B(\xi), J(Y)\rangle & =\langle\hat{B}(\xi), \hat{J}(Y)\rangle \\
\langle\alpha \xi, J(Y)\rangle & =\langle\hat{B}(\xi), \hat{J}(Y)\rangle \\
0 & =\langle\hat{B}(\xi), \hat{J}(Y)\rangle
\end{aligned}
$$

como $\hat{J}$ é invertível, existe $\lambda$ tal que $\hat{B}(\xi)=\lambda \xi$. Portanto $\xi$ é uma direção principal comum aos tensores de Weingarten de $f$ e $\hat{f}$.

CASO II: $r=1$. Neste caso, podemos supor $b_{1} \neq 0$ e $b_{j}=0$ para $j \geq 2$. Em $(i), j=1$ produz para $1 \neq k \neq l \neq 1$

$$
\begin{aligned}
b_{1}^{l}, 0-b_{k}^{l} b_{1} & =0 \\
b_{k}^{l} b_{1} & =0
\end{aligned}
$$

logo, é verdadeiro que $b_{k}^{l}=0$ para $k, l \geq 2$ e $k \neq l$. Mas, de (iii), tomando-se $k, l \geq 2$ e $k \neq l$

$$
\begin{aligned}
\hat{b}_{k} \hat{b}_{l} & =\alpha .0-0.0 \\
& =0
\end{aligned}
$$


Conseqüentemente, existe um índice $m(2 \leq m \leq 2 n)$ tal que $\hat{b}_{m}=0$. Usando $j=m$ e $k=1 \mathrm{em}(i i)$

$$
\begin{aligned}
b_{1}^{m} b_{1}-b_{1}^{1} \cdot 0 & =0 \\
b_{1}^{m} b_{1} & =0
\end{aligned}
$$

expressão da qual tiramos que $b_{1}^{m}=0$ e

$$
\begin{aligned}
B\left(e_{m}\right) & =\sum_{k=1}^{2 n} e_{k}+0 . \xi \\
& =b_{m}^{m} e_{m},
\end{aligned}
$$

isto é, $e_{m}$ é uma direção principal comum a $B$ e $\hat{B}$.

CASO III ( $\mathrm{r}=2$ ). Podemos supor $b_{1}$ e $b_{2}$ não nulos e $b_{j}=0$ para $3 \leq j \leq 2 n$. Para $k=1$ e $j, l \geq 3$ em $(i)$ se transforma em

$$
\begin{aligned}
b_{j}^{l} b_{1}-b_{1}^{l} b_{j} & =0 \\
b_{j}^{l} b_{1}-b_{1}^{l} .0 & =0 \\
b_{j}^{l} b_{1} & =0
\end{aligned}
$$

isto é, $b_{j}^{l}=0$ para $j, l \geq 3$ e $j \neq l$. Disto e de $(i i i)$ tiramos $\hat{b}_{j} \hat{b}_{l}=0$ para $j, l \geq 3$ e $j \neq l$. Portanto, existe um índice $m$ em $(i)(3 \leq m \leq 2 n)$ tal que $\hat{b}_{m}=0$. Fazendo $j=m$ em $(i)$

$$
\begin{aligned}
b_{k}^{m} b_{k}-b_{k}^{k} .0 & =-0 . \lambda_{k} \\
b_{k}^{m} b_{k} & =0
\end{aligned}
$$

o que nos dá $B_{1}^{m}=0, B_{2}^{m}=0 \mathrm{e}$

$$
\begin{aligned}
B\left(e_{m}\right) & =\sum_{k=1}^{2 n} e_{k}+0 . \xi \\
& =b_{m}^{m} e_{m},
\end{aligned}
$$

isto é, $e_{m}$ é uma direção principal comum a $B$ e $\widehat{B}$.

CASO IV: $r>2$. Neste caso podemos supor $b_{1}, b_{2}$ e $b_{3}$ não nulos. Portanto, de $(i)$ temos

$$
b_{2}^{j} b_{1}-b_{1}^{j} b_{2}=0 \quad \operatorname{para} j \geq 2
$$

o que sugere a existência de funções $p_{j}$ para $j=1, \ldots, 2 n$ tais que

$$
b_{1}^{j}=p_{j} b_{1} \quad \text { e } \quad b_{2}^{j}=p_{j} b_{2}
$$


Também de $(i)$

$$
\begin{aligned}
b_{l}^{j} b_{1}-b_{1}^{l} b_{2} & =0, \\
b_{1}\left(b_{j}^{l}-p_{l} b_{j}\right) & =0, \\
b_{j}^{l} & =p_{l} b_{j} \quad \text { para } \quad 3 \leq j \leq 2 n .
\end{aligned}
$$

De $b_{l}^{j}=b_{j}^{l}$ tiramos que $p j=q b_{j}$ para uma certa função $q$, daí obtemos

$$
b_{k}^{j}=q b_{j} b_{k}
$$

para $3 \leq j, k \leq 2 n$. De $(i)$, substituindo $l=1, j=2$ e $k=3$

$$
\begin{aligned}
& b_{2}^{1} b_{3}=b_{3}^{1} b_{2} \\
& b_{2}^{1} b_{3}=b_{2}\left(q b_{1} b_{3}\right) .
\end{aligned}
$$

Como $b_{3} \neq 0$ temos $b_{k}^{j}=q b_{j} b_{k}$ para $1 \leq j \neq k \leq 2 n$.

SUB-CASO $1(r=2 n \operatorname{Se} r=2 n$ então $q \equiv 0$. De fato, suponhamos que $q$ é não nula em algum ponto de $M$. Em $(v)$ substituindo $k=j$

$$
\begin{aligned}
\sum_{l=1}^{2 n} J_{l}^{j} b_{j}^{l} & =0 \\
\sum_{l=1}^{2 n} J_{l}^{j}\left(q b_{j} b_{l}\right) & =0 \\
\left(\sum_{l=1}^{2 n} J_{l}^{j} b_{l}\right) b_{j} & =0
\end{aligned}
$$

portanto $\sum_{l=1}^{2 n} J_{l}^{j} b_{l}=0$ e daí temos $b_{l}=0$, o que é um absurdo. Multiplicando (iii) por $\hat{b}_{l}(l \neq j)$, obtemos

$$
\begin{aligned}
& \alpha b_{j}^{k} \hat{b}_{l}-b_{j} b_{k} \hat{b}_{l}=7 \hat{b}_{l} \hat{b}_{j} \hat{b}_{k} \\
& \alpha b_{j}^{k} \hat{b}_{l}-b_{j} b_{k} \hat{b}_{l}=\alpha b_{l}^{j} \hat{b}_{k}-b_{j} b_{k} \hat{b}_{k} .
\end{aligned}
$$

Mas, usando que $b_{k}^{j}=0$ obtemos para $k \neq j \neq l$

$$
\left(b_{k} \hat{b}_{l}-b_{l} \hat{b}_{k}\right) b_{j}=0
$$

concluindo que $b_{l}=\varepsilon b_{l}$ e (iii) nos dá $\varepsilon^{2}=1$. Como $b_{k}^{j}=0$, (ii) nos produz

$$
\begin{aligned}
b_{j}^{j} b_{k} & =\hat{b}_{k} \lambda_{j} \\
& =\varepsilon b_{k} \lambda_{j}
\end{aligned}
$$


logo tiramos que $b_{j}^{j}=\varepsilon \lambda_{j}$. Voltando a $(i v)$ e fazendo $b_{j}^{j}=\varepsilon \lambda_{j}$ encontramos

$$
(\varepsilon \alpha-\hat{\alpha}) \lambda_{j}=0
$$

Se $\varepsilon \alpha=\hat{\alpha}$, então $B=\varepsilon \widehat{B}$. Em verdade, já sabemos que $b_{k}^{j}=0$ se $1 \leq j \neq k \leq 2 n$, que $b_{j}=\varepsilon \hat{b}_{j}$ e que $b_{j}^{j}=\varepsilon \lambda_{j}$. Portanto, todas as direções principais de $\hat{B}$ em $\mathcal{D}$ são direções principais de $B$. Se $\varepsilon \alpha \neq \hat{\alpha}$ então $\lambda_{j}=$ ), daí teremos os postos (dimensões das imagens) de $B$ e $\hat{B}$ menores que 3 . De Àlgebra Linear segue que

$$
\operatorname{dim}(\operatorname{ker} B+\operatorname{ker} \hat{B})=\operatorname{dim}(\operatorname{ker} B)+\operatorname{dim}(\operatorname{ker} B)-\operatorname{dim}(\operatorname{ker} B \cap \operatorname{ker} \hat{B}),
$$

sendo que $\operatorname{dim}$ significa dimensão e $\operatorname{ker} T$ o núcleo de um certo operador linear $T$. Da fórmula acima e do fato que $\operatorname{dim}(\operatorname{ker} B)$ e $\operatorname{dim}(\operatorname{ker} \hat{B})$ são maiores que $2(n-1)$ tiramos que

$$
\operatorname{dim}(\operatorname{ker} B \cap \operatorname{ker} \hat{B}) \geq 1
$$

Portanto, existe pelo menos uma direção principal comum a $B$ e $\hat{B}$.

SUB-CASO $2(2 \leq r \leq 2 n)$

Neste caso podemos supor $b_{1}=0$. Vejamos que se supusermos $\hat{b}_{1} \neq 0$, substituindo $j=1 \mathrm{em}(i)$

$$
b_{1}^{l} b_{k}=0 \quad \Longrightarrow \quad b_{1}^{l}=0 \quad(l \geq 2)
$$

e substituindo $j=2$ em (iii)

$$
\begin{aligned}
\alpha b_{1}^{l} & =-b_{1} b_{k} \\
b_{1} b_{k} & =0
\end{aligned}
$$

consequentemente $b_{k}=0$ para todo $k$ em $\{2 ; \ldots ; 2 n\}$. Em (ii) substituindo $k=1$

$$
b_{1}^{1} b_{j}=0
$$

como $r \geq 2$, entào $b_{1}^{1}=0$. Mas de $(v)$

$$
\sum_{l=1}^{2 n} J_{l}^{j} b_{k}^{l}=\hat{J}_{k}^{j} \hat{\lambda}_{k}
$$

substituindo $k=1$

$$
0=\hat{J}_{k}^{j} \hat{\lambda}_{k} \quad \Longrightarrow \quad \hat{\lambda}_{1}=0 .
$$

Voltando a ( $i v)$ e fazendo $j=1$ produziremos $b_{1}=0$ o que é um absurdo. Temos assim que $b_{1}=\hat{b}_{1}=0$. Daí uma direção principal comum a $B$ e $\hat{B}$ é bada por $e_{1}$. 
Em [14] é demonstrado que dadas duas imersões isométricas da mesma variedade Riemanniana $M$ em uma forma espacial complexa, com curvaturas holomorfas não nulas, se os tensores de Weingarten possuem uma direção principal em comum então as estruturas complexas induzidas em $M$ pelas imersões isométricas coincidem. Juntando esse fato com a proposição 3.3 .2 e o teorema 3.3 .8 teremos a proposição abaixo

Proposição 3.3.10 Dadas duas imersões sub-Riemannianas $f$ e $\hat{f}$ de $(M, \mathcal{D}, g, \xi)$ em um espaço de formas complexas $N(c)$, com curvatura holomorfa $c$ não nula, então os tensores de Weingaten $B$ e $\hat{B}$ coincidem.

Teorema 3.3.11 Dada uma variedade sub-Riemanniana $(M, \mathcal{D},\langle\rangle,, \xi)$ e imersões isométricas sub-Riemannianas $f_{1}$ e $f_{2}$ de $M$ em $N^{n}(c)$. Se os tensores de Weingarten forem iguais então existe uma isometria $\Psi$ de $N^{n}(c)$ tal que $f_{2}=\Psi f_{1}$.

Demonstração: Usando $\widetilde{R}_{j}$ para denotar o tensor curvature e $J_{j}$ para a estrutura complexa ambos induzidos pela imersão $f_{j}$ em $\mathcal{D}$ e fazendo uso da equação de Gauss, tiramos que

$$
\left\langle\widetilde{R}_{1}(X \wedge Y) Z, W\right\rangle=\left\langle\widetilde{R}_{2}(X \wedge Y) Z, W\right\rangle
$$

e fazendo $Z=Y$ e $W=X$ teremos

$$
\begin{aligned}
\left\langle\widetilde{R}(X, Y) l R_{1}(X \wedge Y) Y, X\right\rangle & =\left\langle R_{2}(X \wedge Y) Y, X\right\rangle \\
\left\langle J_{1} X, Y\right\rangle^{2} & =\left\langle J_{2} X, Y\right\rangle^{2}
\end{aligned}
$$

se $\left\langle J_{1} X, Y\right\rangle=\left\langle J_{1} X, Y\right\rangle$. Da equação de Mainardi-Codazzi temos que $\left\langle\left(D_{\xi}\right)(X)-\left(D_{X}\right)(\xi), y\right\rangle=$ $\frac{c}{4}\langle J X, Y\rangle$ então $J_{1}=J_{2}$. Neste caso, seja $u$ um elemento do fibrado dos referenciais de $\mathrm{M}(B(M))$ tal que $f_{1}(u)$ possa ser completado a um referencial unitário de $N^{n}(c)$. É imediato que $f_{2}(u)$ também pode ser completado a um referencial unitário de $N^{n}(c)$. Portanto, existe uma única isometria $\Psi_{u}: N^{n}(c) \rightarrow N^{n}(c)$ tal que $f_{2}(u)=\Psi_{u} f_{1}(u)$. A aplicaçãoo $u \rightarrow \Psi_{u}$ e constante. Com efeito, de $f_{2}(u)=\Psi(u) f_{1}(u)$ diferenciando teremos

$$
d f_{2}=d L_{\Psi(u)} d f_{1}(X)+d R_{f_{1}(u)} d \Psi(X)
$$

sendo $L_{\Psi(u)}$ e $R_{f_{1}(u)}$ as translações à esquerda e à direita respectivamente. Usando que as formas de conexão $\omega_{k}^{j}$ são invariantes à esquerda temos que

$$
\begin{aligned}
\omega^{j}\left(d R_{f_{1}(u)} d \Psi(X)\right) & =\omega^{j}\left(d f_{2}(X)-d L_{\Psi(u)} d f_{1}(X)\right) \\
& =\omega^{j}(X)-\omega^{j}(X) \\
& =0
\end{aligned}
$$


para $j=1,2, \ldots, 2 n+2$, devido à hipótese $b_{1}=b_{2}$ e portanto $f_{1}^{*} \omega^{2 n+2}=f_{2}^{*} \omega^{2 n+2}$

$$
\begin{aligned}
\omega_{k}^{j}\left(d R_{f_{1}(u)} d \Psi(X)\right) & =\omega_{k}^{j}\left(d f_{2}(X)-d L_{\Psi(u)} d f_{1}(X)\right) \\
& =\omega_{k}^{j}(X)-\omega_{k}^{j}(X) \\
& =0 .
\end{aligned}
$$

Portanto temos que $d R_{f_{1}(u)} d \Psi(X)=0$ e daí $d \Psi(X)=0$. Portanto, a aplicação $\Psi$ é constante.

Teorema 3.3.12 Sejam dadas uma variedade sub-Riemanniana $(M, \mathcal{D}, \xi,\langle\rangle$,$) e i mersões$ sub-Riemannianas isométricas $f_{1}$ e $f_{2}$ de $M$ em $N^{n}(c)$. Se $\operatorname{tr}(J \circ h) \neq 0$ então existe uma isometria $\Psi$ de $N^{n}(c)$ tal que $f_{2}=\Psi f_{1}$.

Demonstração: É imediato que $b(\xi, \xi)$ fica determinado por

$$
b(\xi, \xi)=\frac{1}{\operatorname{tr}(J \circ h)}\left\{\frac{n c}{4}-\operatorname{Ric}(\xi)-\|J \circ h(\xi)\|^{2}\right\}
$$

e consequetemente podemos escrever

$$
\left.b(X, Y)=-\langle J h(x)+J \tau(X), Y\rangle-\langle J h(\xi), X\rangle \theta(Y)+\frac{1}{\operatorname{tr}(J \circ h)}\left\{\frac{n c}{4}-R i c(\xi)-\|J \circ h(\xi)\|^{2}\right\} \theta(X) \theta(Y)\right\}
$$

e pelo teorema anterior temos que existe uma isometria $\Psi$ tal que $f_{2}=\Psi \circ f_{1}$. 


\subsection{O caso sub-Riemanniano tridimensional}

No caso de M ser homegênea no contexto sub-Riemanniano e tridimensional podemos fazer uso de algumas de suas propriedades especiais. Para este caso $\mathrm{N}(\mathrm{c})$ terá dimensão 2 sobre $\mathbb{C}$ e, portanto, existirá uma base local $\left\{e_{1} ; e_{2} ; e_{3} ; e_{4}\right\}$ de $N(c)$ tal que $e_{2}=J e_{1}$ e $e_{4}=J e_{3}$. Podemos assumir que $e_{1}$ é um autovalor de $\tau$. De $\tau \circ J=-J \circ \tau$ teremos que $\left\{e_{1} ; e_{2}\right\}$ diagonaliza $\tau$. Do teorema 2.4.1 temos que

$$
D \xi=\tau+h
$$

com $h: T M \rightarrow \mathcal{D}, \tau: T M \rightarrow \mathcal{D}$ e $\tau(\xi)=0$. As representações matriciais de $\tau$ e $h$ em relação a base acima são dadas por

$$
\tau=\left(\begin{array}{ccc}
\alpha & 0 & 0 \\
0 & -\alpha & 0 \\
0 & 0 & 0
\end{array}\right) \quad h=\left(\begin{array}{ccc}
0 & -\beta & a_{1} \\
\beta & 0 & a_{2} \\
0 & 0 & 0
\end{array}\right) .
$$

Portanto, as representações matriciais de $D \xi$ e $B$ são dadas por

$$
D \xi=\left(\begin{array}{ccc}
\alpha & -\beta & a_{1} \\
\beta & -\alpha & a_{2} \\
0 & 0 & 0
\end{array}\right) \quad B=\left(\begin{array}{ccc}
-\beta & -\alpha & a_{2} \\
-\alpha & -\beta & -a_{1} \\
a_{2} & -a_{1} & \omega(\xi)
\end{array}\right)
$$

Podemos então escrever as seguintes formas de conexão, supondo que $\left\{\theta^{1} ; \theta^{2} ; \theta^{3} ; \theta^{4}\right\}$ é a base dual de $\left\{e_{1} ; e_{2} ; e_{3} ; e_{4}\right\}$ e

$$
D \xi=\omega_{3}^{1} \otimes e_{1}+\omega_{3}^{2} \otimes e_{2}
$$

Então

$$
\begin{aligned}
& \omega_{3}^{1}=\alpha \theta^{1}-\beta \theta^{2}+a_{1} \theta^{3} \\
& \omega_{3}^{2}=\beta \theta^{1}-\alpha \theta^{2}+a_{1} \theta^{3} .
\end{aligned}
$$

Da ortonomalidade de $\left\{e_{1} ; e_{2} ; e_{3}\right\}$ e lembrando que $e_{3}=\xi$ temos

$$
\begin{aligned}
D e_{1} & =\omega_{1}^{2} \otimes e_{2}+\omega_{1}^{3} \otimes \xi \\
D e_{2} & =\omega_{2}^{1} \otimes e_{1}+\omega_{2}^{3} \otimes \xi \\
D \xi & =\omega_{3}^{1} \otimes e_{1}+\omega_{3}^{2} \otimes e_{2} .
\end{aligned}
$$

Pelo Teorema 2.4.1 decorre que

$$
\begin{aligned}
\nabla_{X} e_{1} & =D_{X} e_{1}+\theta(X) h\left(e_{1}\right)+\left\langle D_{X} e_{1}, \xi\right\rangle \xi \\
\nabla_{X} e_{1} & =\omega_{1}^{2}(X) e_{2}+\omega_{1}^{3}(X) \xi-\theta^{3}(X) \beta e_{2}+\omega_{3}^{1}(X) \xi \\
\nabla e_{1} & =\left(\omega_{1}^{2}-\beta \theta^{3}\right) \otimes e_{2} .
\end{aligned}
$$


De forma análoga temos

$$
\nabla e_{2}=\left(\omega_{2}^{1}+\beta \theta^{3}\right) \otimes e_{1}
$$

Ve-se assim que $\eta_{1}^{2}=\omega_{2}^{1}-\beta \theta^{3}$ e $\eta_{2}^{1}=\omega_{2}^{1}+\beta \theta^{3}$ são as formas de conexão sub-Riemannianas de M. Para os campos $e_{1}, e_{2}$ e $\xi$ teremos

$$
\begin{aligned}
{\left[e_{1}, e_{2}\right] } & =\Gamma_{1} e_{1}+\Gamma_{2} e_{2}-2 \beta \xi, \\
{\left[e_{2}, \xi\right] } & =\Gamma e_{1}-\alpha e_{2}+a_{2} \xi, \\
{\left[\xi, e_{1}\right] } & =-\alpha e_{1}+\Gamma e_{2}+a_{1} \xi .
\end{aligned}
$$

Da homogeneidade de $M$ segue que todos os coeficientes são constantes e portanto $\alpha, \beta, a_{1}$ e $a_{2}$ são constantes. Em termos das constantes de estrutura podemos escrever as formas de conexão Riemanniana e sub-Riemanniana

$$
\begin{aligned}
\omega_{2}^{1} & =\Gamma_{1} \theta^{1}+\Gamma_{2} \theta^{2}-(\Gamma+\beta) \theta^{3} \\
\eta_{2}^{1} & =\Gamma_{1} \theta^{1}+\Gamma_{2} \theta^{2}-\Gamma \theta^{3} .
\end{aligned}
$$

Como $\Gamma_{1}, \Gamma_{2}$ e $\Gamma$ são constantes teremos

$$
d \eta_{2}^{1}=\Gamma_{1} d \theta^{1}+\Gamma_{2} d \theta^{2}-\Gamma d \theta^{3} .
$$

Relembrando da seção 2.3 que $k=d \eta_{2}^{1}\left(e_{1}, e_{2}\right), w_{1}=d \eta_{2}^{1}\left(e_{1}, e_{3}\right)$ e $w_{2}=d \eta_{2}^{1}\left(e_{2}, e_{3}\right)$ obteremos as seguintes relações

$$
\begin{aligned}
k & =-\left(\Gamma_{1}\right)^{2}-\left(\Gamma_{2}\right)^{2}-2 \beta \Gamma \\
w_{1} & =-\alpha \Gamma_{1}+\Gamma_{2} \Gamma+a_{1} \Gamma \\
w_{2} & =\Gamma_{1} \Gamma+\Gamma_{2} \alpha+\Gamma a_{2} .
\end{aligned}
$$

As equações de Gauss para o caso de uma imersão isometrica sub-Riemanniana em $N^{2}(c)$ nos dá

$$
\langle\tilde{R}(X, Y) Z, W\rangle=\langle R(X, Y) Z, W\rangle+b(X \wedge Y, Z \wedge W) .
$$

Para a base $\left\{e_{1} ; J e_{1} ; e_{3} ; J e_{3}\right\}$, sendo $e_{3}$ usado para denotar $\xi$, teremos

$$
\begin{aligned}
\left\langle\tilde{R}\left(e_{1}, e_{2}\right) e_{2}, e_{1}\right\rangle & =c \\
\left\langle\tilde{R}\left(e_{1}, e_{2}\right) e_{2}, \xi\right\rangle & =0, \\
\left\langle\tilde{R}\left(e_{1}, \xi\right) e_{1}, e_{2}\right\rangle & =0 \\
\left\langle\tilde{R}\left(e_{1}, \xi\right) e_{1}, \xi\right\rangle & =-\frac{c}{4},
\end{aligned}
$$


usando o fato que

$$
\tilde{R}\left(e_{j}, e_{k}\right) e_{l}=\frac{c}{4}\left\{\left\langle e_{k}, e_{l}\right\rangle e_{j}-\left\langle e_{j}, e_{l}\right\rangle e_{k}+\left\langle J e_{k}, e_{l}\right\rangle J e_{j}-\left\langle J e_{j}, e_{l}\right\rangle J e_{k}+2\left\langle e_{j}, J e_{k}\right\rangle J e_{l}\right\} .
$$

Como a segunda forma $b$ já é conhecida podemos determinar $b\left(e_{j} \wedge e_{k}, e_{l} \wedge e_{m}\right)$ para $j, k, l, m$ variando de 1 a 3 .

$$
\begin{aligned}
b\left(e_{1} \wedge e_{2}, e_{2} \wedge e_{1}\right) & =b\left(e_{1}, e_{2}\right)^{2}-b\left(e_{1}, e_{1}\right) b\left(e_{2}, e_{2}\right) \\
& =\left\langle-J D_{e_{1}} \xi, e_{2}\right\rangle^{2}-\left\langle-J D_{e_{1}} \xi, e_{1}\right\rangle\left\langle-J D_{e_{2}} \xi, e_{2}\right\rangle \\
& =\left\langle h\left(e_{1}\right)+\tau\left(e_{1}\right), e_{1}\right\rangle^{2}\left\langle h\left(e_{1}\right)+\tau\left(e_{1}\right), e_{1}\right\rangle\left\langle h\left(e_{1}\right)+\tau\left(e_{1}\right), e_{2}\right\rangle \\
& =\alpha^{2}-\beta^{2} \\
b\left(e_{1} \wedge e_{2}, e_{2} \wedge \xi\right) & =b\left(e_{1}, e_{2}\right) b\left(e_{2}, \xi\right)-b\left(e_{1}, \xi\right) b\left(e_{2}, e_{2}\right) \\
& =\alpha\left\langle J h(\xi), e_{2}\right\rangle-\left\langle J h(\xi), e_{1}\right\rangle\left\langle J h\left(e_{2}\right), e_{2}\right\rangle \\
& =-\alpha a_{1}+\beta a_{2} \\
b\left(e_{1} \wedge \xi, e_{1} \wedge e_{2}\right)= & b\left(e_{1}, e_{1}\right) b\left(e_{2}, \xi\right)-b\left(e_{1}, e_{2}\right) b\left(e_{1}, \xi\right) \\
= & \beta a_{1}-\alpha a_{2} \\
& \\
b\left(e_{1} \wedge \xi, \xi \wedge e_{1}\right)= & b\left(e_{1}, \xi\right)^{2}-b\left(e_{1}, e_{1}\right) b(\xi, \xi) \\
= & a_{2}^{2}-\beta b(\xi, \xi) .
\end{aligned}
$$

Como as formas de conexão Riemannianas $\omega_{3}^{1}$ e $\omega_{3}^{2}$ são conhecidas, bem como a forma de conexão sub-Riemanniana $\eta_{2}^{1}$ e levando em considereação que as "constantes" de estrutura são constantes poderemos calcular explicitamente o tensor curvatura de M.

Uns cálculos simples, mas trabalhosos, levam-nos às expresões de $R\left(e_{1}, e_{2}\right) e_{2}$ e de $R\left(e_{1}, \xi\right) e_{1}$

$$
R\left(e_{1}, e_{2}\right) e_{2}=\left(\alpha^{2}-3 \beta^{2}+k\right) e_{1}+2\left(\beta a_{2}-\alpha \Gamma_{2}\right) \xi
$$




$$
R\left(e_{1}, \xi\right) e_{1}=\left(a_{2} \alpha-a_{1} \beta+\alpha \Gamma_{1}-\Gamma_{2} \Gamma+a_{1} \Gamma\right) e_{2}+\left(\alpha^{2}+a_{2} \Gamma_{1}-\left(a_{1}\right)^{2}\right) \xi
$$

Portanto, voltanto às equações de Gauss teremos

G1) $\left\langle R\left(e_{1}, e_{2}\right) e_{2}, e_{1}\right\rangle=\left\langle\widetilde{R}\left(e_{1}, e_{2}\right) e_{2}, e_{1}\right\rangle-b\left(e_{1} \wedge e_{2}, e_{2} \wedge e_{1}\right)$

$$
\begin{aligned}
\alpha^{2}-3 \beta^{2}+k & =c-\alpha^{2}+\beta^{2} \\
k & =c+4 \beta^{2}-2 \alpha^{2}
\end{aligned}
$$

G2) $\left\langle R\left(e_{1}, e_{2}\right) e_{2}, \xi\right\rangle=\left\langle\widetilde{R}\left(e_{1}, e_{2}\right) e_{2}, \xi\right\rangle-b\left(e_{1} \wedge e_{2}, e_{2} \wedge \xi\right)$

$$
\begin{aligned}
2\left(\beta a_{2}-\alpha \Gamma_{2}\right) & =-\beta a_{2}+\alpha a_{1} \\
\alpha\left(a_{1}+2 \Gamma_{2}\right) & =3 \beta a_{2}
\end{aligned}
$$

G3) $\left\langle R\left(e_{1}, \xi\right) e_{1}, e_{2}\right\rangle=\left\langle\widetilde{R}\left(e_{1}, \xi\right) e_{1}, e_{2}\right\rangle-b\left(e_{1} \wedge \xi, e_{1} \wedge e_{2}\right)$

$$
\begin{aligned}
a_{2} \alpha-a_{1} \beta+\alpha \Gamma_{1}-\Gamma_{2} \Gamma+a_{1} \Gamma & =\alpha a_{2}-\beta a_{1} \\
\alpha \Gamma_{1}-\Gamma_{2} \Gamma+\Gamma a_{1} & =0
\end{aligned}
$$

G4) $\left\langle R\left(e_{1}, \xi\right) e_{1}, \xi\right\rangle=\left\langle\widetilde{R}\left(e_{1}, \xi\right) e_{1}, \xi\right\rangle-b\left(e_{1} \wedge \xi, e_{1} \wedge \xi\right)$

$$
\begin{aligned}
\alpha^{2}-a_{2} \Gamma_{1}-\left(a_{1}\right)^{2} & =-\frac{c}{4}-\left(a_{2}\right)^{2}+\beta \omega(\xi) \\
\alpha^{2}+a_{2} \Gamma_{1}+\left(a_{2}\right)^{2}-\left(a_{1}\right)^{2}-\beta \omega(\xi)+\frac{c}{4} & =0 .
\end{aligned}
$$

Antes de vermos quais são as equações de Mainardi-Codazzi vejamos qual a expressão de $b$ em relação aos 1 -formas $\theta_{1}, \theta_{2}$ e $\theta$. Sabemos que

$$
b(X, Y)=-\left\langle J D_{X} \xi, Y\right\rangle-\left\langle J D_{\xi} \xi, X\right\rangle \theta(Y)+s(\xi) \theta(X) \theta(Y)
$$

usando a representação matricial de $B$ chega-se a $b=\beta \theta^{1} \otimes \theta^{1}-\alpha \theta^{1} \otimes \theta^{2}+a_{2} \theta^{1} \otimes \theta-\alpha \theta^{2} \otimes \theta^{1}+\beta \theta^{2} \otimes \theta^{2}-a_{1} \theta^{2} \otimes \theta+a_{2} \theta \otimes \theta^{1}-a_{1} \theta \otimes \theta^{2}+s(\xi) \theta \otimes \theta$ e portanto podemos calcular as derivadas covariantes de $b$ nas varias direções:

$$
\begin{aligned}
\left(D_{e_{1}} b\right)\left(e_{2}, e_{1}\right) & =-b\left(D_{e_{1}} e_{2}, e_{1}\right)-b\left(D_{e_{1}} e_{1}, e_{2}\right) \\
& =-\omega_{2}^{1}\left(e_{1}\right) b\left(e_{1}, e_{1}\right)-\omega_{2}^{3}\left(e_{1}\right) b\left(e_{1}, \xi\right)-\omega_{1}^{2}\left(e_{1}\right) b\left(e_{2}, e_{2}\right)-\omega_{1}^{3}\left(e_{1}\right) b\left(e_{2}, \xi\right) \\
& =-\Gamma_{1} \beta+\beta a_{2}+\Gamma_{1} \beta-\alpha a_{1} \\
& =\beta a_{2}-\alpha a_{1} .
\end{aligned}
$$


De forma análoga

$$
\begin{aligned}
& \left(D_{e_{2}} b\right)\left(e_{1}, e_{1}\right)=-2\left(\alpha \Gamma_{2}+\beta a_{2}\right) \\
& \left(D_{e_{1}} b\right)\left(e_{2}, e_{2}\right)=2\left(\alpha \Gamma_{1}-\beta a_{1}\right) \\
& \left(D_{e_{2}} b\right)\left(e_{1}, e_{2}\right)=\beta\left(a_{1}-a_{2}\right) \\
& \left(D_{e_{1}} b\right)\left(e_{2}, \xi\right)=a_{2} \Gamma_{1}+\beta \omega(\xi)+a_{1} \alpha-\beta^{2} \\
& \left(D_{e_{2}} b\right)\left(e_{1}, \xi\right)=-a_{1} \Gamma_{2}-\beta \omega(\xi)+\beta^{2}-\alpha a_{2} \\
& \left(D_{e_{1}} b\right)\left(\xi, e_{2}\right)=\alpha^{2}+\beta \omega(\xi)+a_{1} \beta-\Gamma_{1} a_{2} \\
& \left(D_{\xi} b\right)\left(e_{1}, e_{2}\right)=\left(a_{2}\right)^{2}-\left(a_{1}\right)^{2} .
\end{aligned}
$$

Estamos aptos a escrever as equações de Mainardi-Codazzi

$\mathrm{MC} 1)\left\langle\tilde{R}\left(e_{1}, e_{2}\right) \eta, \xi\right\rangle=\left(D_{e_{1}} b\right)\left(e_{2}, \xi\right)-\left(D_{e_{2}} b\right)\left(e_{1}, \xi\right)$

$$
a_{2}\left(\Gamma_{1}+\alpha\right)+a_{1}\left(\Gamma_{2}+\alpha\right)+2 \beta(\beta+\omega(\xi))=0
$$

$\mathrm{MC} 2)\left\langle\tilde{R}\left(e_{1}, e_{2}\right) \eta, e_{1}\right\rangle=\left(D_{e_{1}} b\right)\left(e_{2}, e_{1}\right)-\left(D_{e_{2}} b\right)\left(e_{1}, e_{1}\right)$

$$
3 \beta a_{2}+\alpha\left(2 \Gamma_{2}-a_{1}\right)=0
$$

MC3 $)\left\langle\tilde{R}\left(e_{1}, e_{2}\right) \eta, e_{2}\right\rangle=\left(D_{e_{1}} b\right)\left(e_{2}, e_{2}\right)-\left(D_{e_{2}} b\right)\left(e_{1}, e_{2}\right)$

$$
2 \alpha \Gamma_{1}+\beta\left(a_{2}-3 a_{1}\right)=0
$$

MC4) $\left\langle\tilde{R}\left(e_{1}, \xi\right) \eta, e_{2}\right\rangle=\left(D_{e_{1}} b\right)\left(\xi, e_{2}\right)-\left(D_{\xi} b\right)\left(e_{1}, e_{2}\right)$

$$
\alpha^{2}-\beta \omega(\xi)+a_{1} \beta-\Gamma_{1} a_{2}+\left(a_{1}\right)^{2}-\left(a_{2}\right)^{2}-\frac{c}{4}=0 .
$$

Passemos à análise dos casos nos quais $\xi$ gera um fluxo geodésico. Neste caso teremos $D_{\xi} \xi=0$ e como consequência $a_{1}=a_{2}=0$. Substituindo os valores de $a_{1}$ e $a_{2}$ nas equações acima obteremos o seguinte conjunto de equações 
(i) $k=-\left(\Gamma_{1}\right)^{2}-\left(\Gamma_{2}\right)^{2}-2 \beta \Gamma$;

(ii) $k=c+4 \beta^{2}-2 \alpha^{2}$;

(iii) $\alpha \Gamma_{2}=0$;

(iv) $\Gamma_{2} \Gamma-\alpha \Gamma_{1}=0$;

(v) $\alpha^{2}-\beta \omega(\xi)-\frac{c}{4}=0 ;$

(vi) $\beta(\beta+\omega(\xi))=0$;

(vii) $\alpha \Gamma_{2}=0$;

(viii) $\alpha \Gamma_{1}=0$;

(ix) $\alpha^{2}-\beta \omega(\xi)-\frac{c}{4}=0$.

Ainda com esta restrição existirá diversos casos para analisarmos. Passemos então à análise desses casos:

I - $\beta=0$ ( $\mathcal{D}$ é integrável) Substituindo $\beta=0$ nas equações acima obteremos o seguinte conjunto de equações

(i) $k=-\left(\Gamma_{1}\right)^{2}-\left(\Gamma_{2}\right)^{2}$;

(ii) $k=c-2 \alpha^{2}$;

(iii) $\alpha \Gamma_{2}=0$;

(iv) $\Gamma_{2} \Gamma-\alpha \Gamma_{1}=0$;

(v) $\alpha^{2}-\frac{c}{4}=0$;

(vi) $\alpha \Gamma_{2}=0$;

(vii) $\alpha \Gamma_{1}=0$.

I.i $\beta=0$ e $\alpha=0$, isto é, $\mathcal{D}$ é integrável com torção nula. Neste caso, teremos

$\Gamma_{1}=\Gamma_{2}=0, k=0$ e $c=0$. Portanto, podemos escrever os colchetes da seguinte forma

$$
\begin{aligned}
{\left[e_{1}, e_{2}\right] } & =0 \\
{\left[e_{2}, \xi\right] } & =\Gamma e_{1} \\
{\left[\xi, e_{1}\right] } & =\Gamma e_{2} .
\end{aligned}
$$


Logo concluímos que se $\Gamma=0$ então $M=\mathbb{C} \times \mathbb{R}$ e se $\Gamma \neq 0$ então $M=E(2)$ (o grupo dos movimentos rígidos do plano Euclidiano).

I.ii $\beta=0$ e $\alpha \neq 0$, isto é, $\mathcal{D}$ integrável com torção não nula. Neste caso uma análise nas equações de Gauss e Mainardi-Codazzi nos mostram que: $\Gamma_{1}=\Gamma_{2}=0$, $k=0$ e $c \geq 0$

$$
\begin{aligned}
{\left[e_{1}, e_{2}\right] } & =0 \\
{\left[e_{2}, \xi\right] } & =\Gamma e_{1}-\alpha e_{2} \\
{\left[\xi, e_{1}\right] } & =-\alpha e_{1}+\Gamma e_{2}
\end{aligned}
$$

Neste caso fazendo uma mudança de base $f_{1}=\frac{e_{1}+e_{2}}{\sqrt{2}}$ e $f_{2}=\frac{e_{1}-e_{2}}{\sqrt{2}}$ obteremos

$$
\begin{aligned}
{\left[f_{1}, f_{2}\right] } & =0 \\
{\left[f_{2}, \xi\right] } & =(\Gamma-\alpha) f_{1} \\
{\left[\xi, f_{1}\right] } & =(\alpha+\Gamma) f_{2} .
\end{aligned}
$$

Então $M=\mathbb{C} \times \mathbb{R}$ produto semi-direto da álgebra abeliana $\mathbb{C}$ por $\mathbb{R}$ ou o grupo Euclidiano $E(2)$ ou o grupo dos movimentos rígidos do plano hiperbólico, dependendo da relação entre $\alpha$ e $\Gamma$.

II $-\beta \neq 0$. O caso de contacto.

II.i - $\beta \neq 0$ e $\alpha=0$ ( contacto sem torção).

II.i.1 - Contacto sem torção e $\Gamma=0$. Para este caso uma análise nos mostra que $k=-\Gamma_{1}^{2}-\Gamma_{2}^{2} \leq 0$. Portanto, de $k=c+4 \beta^{2}$ segue que $c \leq-4 \beta^{2}<0$

$$
\begin{aligned}
{\left[e_{1}, e_{2}\right] } & =\Gamma_{1} e_{1}+\Gamma_{2} e_{2}-2 \beta \xi \\
{\left[e_{2}, \xi\right] } & =0 \\
{\left[\xi, e_{1}\right] } & =0
\end{aligned}
$$

Se $\Gamma_{1}=\Gamma_{2}=0$ então $k=0$ e o grupo correspondente é o Grupo de Heisenberg $H^{3}$. Se $\Gamma_{1} \neq 0$ e/ou $\Gamma_{2} \neq 0$ então $M=A(1) \oplus \mathbb{R}$, sendo $A(1)$ usado para denotar grupo afim da reta real. 
II.i.2 - Contacto sem torção e $\Gamma \neq 0$

$$
\Gamma_{1}=\Gamma_{2}=0, \quad k=-2 \beta \Gamma \mathrm{e} \quad k=c .
$$

Então $\Gamma=k / 2 \beta \mathrm{e}$

$$
\begin{aligned}
{\left[e_{1}, e_{2}\right] } & =-2 \beta \xi \\
{\left[e_{2}, \xi\right] } & =-\frac{k}{2 \beta} e_{1} \\
{\left[\xi, e_{1}\right] } & =-\frac{k}{2 \beta} e_{2} .
\end{aligned}
$$

Comparando com o segundo capítulo teremos as seguintes casos:

\begin{tabular}{|c|c|c|}
\hline$k$ & $c$ & $M$ \\
\hline$k>0$ & $c>-4 \beta^{2}$ & $S U(2)$ \\
\hline $\mathrm{k}=0$ & $c=4 \beta^{2}$ & $H^{3}$ \\
\hline$k<0$ & $c<-4 \beta^{2}$ & $S U(1,1)$ \\
\hline
\end{tabular}

II.ii - $\beta \neq 0$ e $\alpha \neq 0$ Contacto com torção não nula. Neste caso uma análise nas equações de Gauss e Mainardi-Codazzi nos dão $\Gamma_{1}=\Gamma_{2}=0$ e $\Gamma=-\frac{k}{2 \beta}$. Portanto, escreve-se da seguinte forma o colchete da base ortonormal $\left\{e_{1} ; e_{2} ; \xi\right\}$

$$
\begin{aligned}
{\left[e_{1}, e_{2}\right] } & =-2 \beta \xi \\
{\left[e_{2}, \xi\right] } & =-\frac{k}{2 \beta} e_{1}-\alpha e_{2} \\
{\left[\xi, e_{1}\right] } & =-\alpha e_{1}-\frac{k}{2 \beta} e_{2} .
\end{aligned}
$$

Podemos supor $\alpha$ e $\beta$ positivos. Temos então três casos que são mostrados na tabela abaixo e foram classificados em [5] e tratados de uma forma mais geométrica em [2]:

\begin{tabular}{|c||c|c|c|}
\hline caso & $k$ & $c$ & $M$ \\
\hline i & $k>2 \alpha \beta$ & $c>-4 \beta(\beta+\alpha)$ & $S U(2)$ \\
\hline ii & $k=2 \alpha \beta$ & $c=-4 \beta(\beta+\alpha)$ & $E(2)$ \\
\hline iii & $0<k<2 \alpha \beta$ & $c<-4 \beta(\beta+\alpha)$ & $S U(1,1)$ \\
\hline
\end{tabular}




\subsection{Imersões isométricas de $S_{r}^{3}$ em $N(c)$}

Primeiramente trataremos o caso de imersões da esfera de raio $r\left(S_{r}^{3}\right)$ em $\mathbb{C}^{2}, \mathbb{C I}^{2}$ e $\mathbb{C H}^{2}$.

Imersão de $S_{r}^{3}$ em $\mathbb{C}^{2}$ :

$S_{r}^{3}$ esta naturalmente imersa em $\mathbb{C}^{2}$. De fato, por definição

$$
S_{r}^{3}=\left\{\left(x_{1}, x_{2}, x_{3}, x_{4}\right) \in \mathbb{R}^{4}: x_{1}^{2}+x_{2}^{2}+x_{3}^{2}+x_{4}^{2}=r^{2}\right\} .
$$

É suficiente então definir $f$ da seguinte forma: façamos $z_{1}=x_{1}+i x_{2}$ e $z_{2}=x_{3}+i x_{4}$, e portanto podemos identificar $S_{r}^{3}=\left\{\left(z_{1}, z_{2}\right) \in \mathbb{C}^{2}: z_{1} \overline{z_{1}}+z_{2} \overline{z_{2}}=r^{2}\right\}$ e finalmente definir

$$
f\left(x_{1}, x_{2}, x_{3}, x_{4}\right)=\left(z_{1}, z_{2}\right) \text {. }
$$

Um cálculo simples nos mostra que se definirmos $\omega$ como sendo a um forma complexa dada por

$$
\omega=\overline{z_{1}} d z_{1}+\overline{z_{2}} d z_{2}+z_{1} d \overline{z_{1}}+z_{2} d \overline{z_{2}}
$$

teremos que no ponto $z=\left(z_{1}, z_{2}\right)$

$$
T_{z} S_{r}^{3}=\left\{v \in \mathbb{C}^{2}: \omega_{z}(v)=0\right\} .
$$

Daí teremos que $f_{1}=\frac{1}{r}\left(-\overline{z_{2}}, \overline{z_{1}}\right)$ e $f_{3}=\frac{i}{r}\left(z_{1}, z_{2}\right)$ são vetores tangentes à esfera. Como $-i f_{3}$ é normal à esfera $S_{r}^{3}$ a distribuição holomorfa $\mathcal{D}$ é dada por

$$
\mathcal{D}(z)=\left\{\alpha f_{1}: \alpha \in \mathbb{C}\right\} .
$$

Podemos tomar o seguinte referencial ortonormal $\left\{f_{1}, f_{2}=i f_{1}, f_{3}\right\}$ e $\{\omega,-i \omega, \theta\}$ o coreferencial associado. Como neste caso $\eta$ o vetor normal é dado por $\eta=\frac{1}{r}\left(z_{1}, z_{2}\right)$

$$
\begin{aligned}
d \eta & =\frac{1}{r}\left(d z_{1}, d z_{2}\right) \\
d \eta & =\frac{1}{r}\left(\theta^{1} \otimes f_{1}+\theta^{2} \otimes f_{2}+\theta^{3} \otimes f_{3}\right) .
\end{aligned}
$$

Sendo $\left\{e_{1} ; e_{2}\right\}$ a base canônica do $\mathbb{C}^{2}$, poderemos escrever

$$
\begin{aligned}
f_{1}=\frac{1}{r}\left(-\overline{z_{2}} e_{1}+\overline{z_{1}} e_{2}\right) \omega & =\frac{1}{r}\left(z_{2} d z_{1}+z_{1} d z_{2}\right) \\
f_{3}=-\frac{i}{r}\left(z_{1} e_{1}+z_{2} e_{2}\right) \eta & =\frac{1}{r}\left(\overline{z_{1}} d z_{1}+\overline{z_{2}} d z_{2}\right)
\end{aligned}
$$


3.5 Imersões isométricas de $S_{r}^{3}$ em $N(c)$

$$
\begin{gathered}
d f_{1}=\frac{1}{r^{2}}\left(z_{1} d \overline{z_{1}}+z_{2} \overline{z_{2}}\right) \otimes+f_{1} \frac{1}{r^{2}}\left(\overline{z_{2}} d \overline{z_{1}}-\overline{z_{2}} d \overline{z_{2}}\right) \otimes f_{3} \\
d f_{3}=\frac{1}{r^{2}}\left(-z_{2} d z_{1}+z_{2} d z_{2}\right) \otimes f_{1}+\frac{1}{r^{2}}\left(\overline{z_{2}} d z_{1}+\overline{z_{2}} d z_{2}\right) \otimes f_{3} \\
d f_{1}=-\frac{i}{r} \theta \otimes f_{1}-\frac{1}{r} \bar{\omega} \otimes f_{3} \\
d f_{3}=\frac{1}{r} \omega \otimes f_{1}+\frac{i}{r} \bar{\omega} \otimes f_{3}
\end{gathered}
$$

e logo tiramos que

$$
\begin{aligned}
d \theta & =-\frac{i}{r} \omega \wedge \bar{\omega} \\
d \omega & =-\frac{2 i}{r} \bar{\omega} \wedge \theta .
\end{aligned}
$$

É fácil verificar que $\theta$ restrita ao espaço tangente à esfera é uma 1-forma real . escrevendo $\omega=\theta^{1}+i \theta^{2}$, sendo $\theta^{1}$ e $\theta^{2}$ formas reais, teremos

$$
\begin{aligned}
d \theta^{1} & =-\frac{2}{r} \theta^{2} \wedge \theta \\
d \theta^{2} & =-\frac{2}{r} \theta \wedge \theta^{1} \\
d \theta & =-\frac{2}{r} \theta^{1} \wedge \theta^{2}
\end{aligned}
$$

e logo as equações podem ser reescritas como

$$
\begin{aligned}
d \theta^{1}+\frac{-\theta}{r} \wedge \theta^{2}+\frac{\theta^{2}}{r} \wedge \theta & =0 \\
d \theta^{2}+\frac{-\theta}{r} \wedge \theta^{1}+\frac{-\theta^{1}}{r} \wedge \theta & =0 \\
d \theta^{1}+\frac{-\theta^{2}}{r} \wedge \theta^{1}+\frac{-\theta^{1}}{r} \wedge \theta^{2} & =0
\end{aligned}
$$

expressão da qual tiramos que $\omega_{3}^{2}=\frac{-1}{r} \theta^{1}$ e $\omega_{3}^{1}=\frac{1}{r} \theta^{2}$ e portanto

$$
D \xi=\frac{1}{r} \theta^{2} \otimes f_{1}-\frac{1}{r} \theta^{1} \otimes f_{2} .
$$


Podemos então calcular a representação matricial de $D \xi$

$$
\begin{array}{r}
D_{f_{1}} \xi=-\frac{1}{r} \theta^{1}\left(f_{1}\right) f_{2}=-\frac{1}{r} f_{2} \\
D_{f_{2}} \xi=\frac{1}{r} \theta^{2}\left(f_{2}\right) f_{1}=\frac{1}{r} f_{1}
\end{array}
$$

e portanto $\tau=0$ e $h$ é representado por

$$
h=\left[\begin{array}{ccc}
0 & \frac{1}{r} & 0 \\
\frac{-1}{r} & 0 & 0 \\
0 & 0 & 0
\end{array}\right] .
$$

Um cálculo simples nos leva a $k=\frac{2}{r^{2}}$.

Imersão de $S_{r}^{3}$ em $\mathbb{C P}^{2}\left(\frac{4}{R^{2}}\right)$ :

Antes de começarmos imersões em $\mathbb{C P}^{2}$ relembremos que

$$
S^{1} \longrightarrow S^{5} \longrightarrow \mathbb{C I P}^{2}
$$

é uma fibração de Hopf. Sendo $z=\left(z_{1}, z_{2}, z_{3}\right)$ as coordenadas usuais do $\mathbb{C}^{3}$ teremos

$$
T_{z} S^{5}=\left\{v \in \mathbb{C}^{3}: \operatorname{Re}\langle z, v\rangle=0\right\}
$$

A distribuição $\mathcal{D}_{z}$ é obtida tomando o núcleo da 1- forma complexa

$$
\omega_{z}=\overline{z_{1}} d z_{1}+\overline{z_{2}} d z_{2}+\overline{z_{3}} d z_{3}
$$

A esfera $S_{r}^{3}$ pode ser imersa em $\mathbb{C P}^{2}\left(\frac{4}{R^{2}}\right)$ da seguinte forma: Seja $\widetilde{S}$ a hipervariedade de $S_{R}^{5}$ definida por

$$
\widetilde{S}=\left\{\left(z_{1}, z_{2}, z_{3}\right) \in S_{R}^{5}: \overline{z_{1}} z_{1}+\overline{z_{2}} z_{2}=\frac{r^{2}}{R^{2}-r^{2}} \overline{z_{3}} z_{3}\right\}
$$

Podemos projetar, por motivos já explicados, $\widetilde{S}$ em $\mathbb{C P}^{2}\left(\frac{4}{R^{2}}\right)$ e obter uma hipervariedade $S$ de $\mathbb{C P P}^{2}\left(\frac{4}{R^{2}}\right)$

$$
S=\pi(\widetilde{S})=\left\{\left[\left(z_{1}, z_{2}, z_{3}\right)\right] \in \mathbb{C I P}^{2}\left(\frac{4}{R^{2}}\right): \overline{z_{1}} z_{1}+\overline{z_{2}} z_{2}=r^{2} e \overline{z_{3}} z_{3}=R^{2}-r^{2}\right\}
$$


Portanto, definindo $\tilde{f}: S_{r}^{3} \longrightarrow S_{R}^{5}$ por

$$
\widetilde{f}\left(z_{1}, z_{2}\right)=\frac{1}{R}\left(z_{1}, z_{2}, \sqrt{R^{2}-r^{2}}\right)
$$

teremos uma imersão isométrica de $S_{r}^{3}$ em $S_{R}^{5}$. Daí $f=\pi \circ \tilde{f}$ nos dá uma imersão isométrica de $S_{r}^{3}$ em $\mathbb{C P}^{2}\left(\frac{4}{R^{2}}\right)$.

Começaremos o cálculo de $\tau, h$ e $b$ para esta imersão. Consideremos inicialmente o seguinte referencial unitário do $\mathbb{C}^{3}$ restrito a $S_{r}^{5}$

$$
\begin{aligned}
\tilde{f}_{1} & =\left(-\overline{z_{2}}, \overline{z_{1}}, 0\right) \\
\tilde{f}_{2} & =\frac{\alpha}{r R}\left(z_{1}, z_{2},-\frac{r^{2}}{\alpha}\right) \\
\tilde{f}_{3} & =\frac{1}{R}\left(z_{1}, z_{2}, \alpha\right),
\end{aligned}
$$

sendo $\alpha$ uma constante dada por $\alpha=\sqrt{R^{2}-r^{2}}$. Sabemos que o referencial $\left\{\widetilde{f}_{1}, \widetilde{f}_{2}, \widetilde{f}_{3}\right\}$ satifaz

$$
\begin{aligned}
& d \tilde{f}_{1}=\omega_{1}^{1} \otimes \tilde{f}_{1}+\omega_{1}^{2} \otimes \tilde{f}_{2}+\omega_{1}^{3} \otimes \tilde{f}_{3} \\
& d \tilde{f}_{2}=\omega_{2}^{1} \otimes \tilde{f}_{1}+\omega_{2}^{2} \otimes \tilde{f}_{2}+\omega_{2}^{3} \otimes \tilde{f}_{3} \\
& d \tilde{f}_{3}=\omega_{3}^{1} \otimes \tilde{f}_{1}+\omega_{3}^{2} \otimes \tilde{f}_{2}+\omega_{3}^{3} \otimes \tilde{f}_{3}
\end{aligned}
$$

para certas 1-formas $\omega_{k}^{j}$, temos então usando os resultados da primeira seção a derivada covariante $\tilde{D}$ do $\mathbb{C P}^{2}\left(\frac{4}{R^{2}}\right)$

$$
\begin{aligned}
& \tilde{D} f_{1}=\left(\omega_{1}^{1}-\omega_{3}^{3}\right) \otimes f_{1}+\omega_{1}^{2} \otimes f_{2} \\
& \tilde{D} f_{2}=\omega_{2}^{1} \otimes f_{1}+\left(\omega_{2}^{2}-\omega_{3}^{3}\right) \otimes f_{2} .
\end{aligned}
$$

Restrito à esfera $S_{R}^{5}$ temos que $d \tilde{f}_{2}=\frac{\alpha}{r^{2}} d \tilde{f}_{3}$ e consequetemente

$$
\omega_{2}^{3}=\frac{\alpha}{r} \omega_{3}^{3} \quad \omega_{2}^{2}=\frac{\alpha}{r} \omega_{3}^{2} \quad \omega_{2}^{1}=\frac{\alpha}{r} \omega_{3}^{1} .
$$

Denotando por $\left\{\theta^{1} ; \theta^{2} ; \theta^{3}\right\}$ o referencial dual a $\left\{\tilde{f}_{1} ; \tilde{f}_{2} ; \tilde{f}_{3}\right\}$ e por $\left\{e_{1} ; e_{2} ; e_{3}\right\}$ a base canônica do $\mathbb{C}^{3}$ poderemos escrever

$$
\begin{aligned}
d \tilde{f}_{1} & =\frac{1}{r}\left(-d \overline{z_{2}} \otimes e_{1}+d \overline{z_{1}} \otimes e_{2}\right) \\
& =\left(\frac{\alpha}{r R} \overline{\theta^{2}}+\frac{1}{R} \overline{\theta^{3}}\right) \otimes f_{1}-\frac{\alpha}{r R} \overline{\theta^{1}} \otimes f_{2}-\frac{1}{R} \overline{\theta^{1}} \otimes f_{3}
\end{aligned}
$$




$$
\begin{aligned}
d \tilde{f}_{2} & =\frac{\alpha}{r R}\left(d z_{1} \otimes e_{1}+d z_{2} \otimes e_{2}\right. \\
& =\left(\frac{\alpha}{r R} \theta^{1}\right) \otimes f_{1}+\left(\frac{\alpha^{3}}{r R^{3}} \theta^{2}+\frac{\alpha^{2}}{R^{3}} \theta^{3}\right) \otimes f_{2}+\left(\frac{a f^{2}}{R^{3}} \theta^{2}+\frac{r \alpha}{R^{3}} \theta^{3}\right) \otimes f_{3} \\
d \tilde{f}_{3} & =\frac{1}{R}\left(d z_{1} \otimes e_{1}+d z_{2} \otimes e_{2}\right. \\
& =\left(\frac{1}{R} \theta^{1}\right) \otimes f_{1}+\left(\frac{\alpha^{2}}{r R^{3}} \theta^{2}+\frac{r \alpha}{R^{3}} \theta^{3}\right) \otimes f_{2}+\left(\frac{r \alpha}{R^{3}} \theta^{2}+\frac{r^{2}}{R^{3}} \theta^{3}\right) \otimes f_{3} .
\end{aligned}
$$

Definindo $\theta=i\left(\frac{\alpha}{r R} \theta^{2}+\frac{1}{R} \theta^{3}\right)$ poderemos escrever para as formas de conexão

$$
\begin{array}{cc}
\omega_{1}^{1}=-i \theta & \omega_{2}^{1}=\frac{\alpha}{r R} \theta^{1} \\
\omega_{2}^{2}=-i \frac{\alpha^{2}}{R^{2}} \theta & \omega_{3}^{3}=i \frac{r^{2}}{R^{2}} \theta
\end{array}
$$

podemos escrever

$$
\begin{aligned}
& \tilde{D} f_{1}=i \frac{a f^{2}}{R^{2}} \theta \otimes f_{1}+\left(-\frac{a f}{r R} \overline{\theta^{1}}\right) \otimes f_{2} \\
& \tilde{D} f_{2}=i \frac{a f}{R} \theta^{1} \otimes f_{1}+(-i \theta) \otimes f_{2} .
\end{aligned}
$$

A distribuição holomorfa $\mathcal{D}$ desta imersão da esfera é dada por $\mathcal{D}=\left\{\mu f_{1}: \mu \in \mathbb{C}\right\}$. Daí voltando a notação $\eta=f_{2}$ e $\xi=-J\left(f_{2}\right)$

$$
\begin{aligned}
\tilde{D} \eta & =\frac{\alpha}{r R} \theta^{1} \otimes f_{1}+\theta \otimes \xi \\
\tilde{D} \xi & =-\frac{\alpha}{r R} \theta^{1} \otimes\left(i f_{1}\right)+(-\theta) \otimes \eta
\end{aligned}
$$

e disto tiramos que $D \xi=-\frac{\alpha}{r R} \theta^{1} \otimes\left(i f_{1}\right)$. Temos assim tudo que necessitamos para calcular a representação matricial de $D \xi$

$$
\begin{aligned}
D_{f_{1}} \xi & =-\frac{\alpha}{r R}\left(i f_{1}\right) \\
D_{i f_{1}} \xi & =\frac{\alpha}{r R} f_{1}
\end{aligned}
$$

e daí concluímos que $\tau=0 \mathrm{e}$

$$
h=\left[\begin{array}{cc}
0 & -\frac{\alpha}{r R} \\
\frac{\alpha}{r R} & 0
\end{array}\right]
$$


Além disso $b(\xi, \xi)=-\left\langle\tilde{D}_{\xi} \eta, \xi\right\rangle=-\theta(\xi)=\frac{\alpha}{r R} \neq 0$. Temos então pelo teorema anterior que qualque outra imersão da esfera $S_{r}^{3}$ em $\mathbb{C P P}^{2}\left(\frac{4}{R^{2}}\right)$ tal que $b(\xi, \xi)$ for não nulo diferirá desta imersão por uma isometria de $\mathbb{C P}^{2}\left(\frac{4}{R^{2}}\right)$. Em verdade, $b(\xi, \xi) \neq 0$ implicará no traço de $h$ não-nulo, portanto, podemos aplicar um dos teoremas de rigidez.

Imersão de $S_{r}^{3}$ em $\mathbb{C H}^{2}\left(\frac{4}{R^{2}}\right)$ :

Relembremos que $h(z, w)=z_{1} \bar{w}_{1}+z_{2} \bar{w}_{2}-z_{3} \bar{w}_{3}, Q_{R}^{5}=\left\{z \in \mathbb{C}^{3} \quad: \quad h(z, z)=-R^{2} \quad\right\}$ e que

$$
S^{1} \longrightarrow Q_{R}^{5} \longrightarrow \mathbb{C H}^{2}\left(\frac{4}{R^{2}}\right)
$$

é uma fibração de Hopf. Sendo $z=\left(z_{1}, z_{2}, z_{3}\right)$ as coordenadas usuais do $\mathbb{C}^{3}$ teremos

$$
T_{z} Q_{R}^{5}=\left\{v \in \mathbb{C}^{3}: h(z, v)+h(v, z)=0\right\}
$$

a distribuição $\mathcal{D}_{z}$ é obtida tomando o núcleo da 1 - forma complexa

$$
\omega_{z}=\overline{z_{1}} d z_{1}+\overline{z_{2}} d z_{2}-\overline{z_{3}} d z_{3}
$$

esfera $S_{r}^{3}$ pode ser imersa em $\mathbb{C H}^{2}\left(\frac{4}{R^{2}}\right)$ da seguinte forma: Seja $\widetilde{S}$ a hipervariedade de $Q_{R}^{5}$ definida por

$$
\widetilde{S}=\left\{\left(z_{1}, z_{2}, z_{3}\right) \in Q_{R}^{5}: z_{1} \overline{z_{1}}+z_{2} \overline{z_{2}}=\frac{r^{2}}{R^{2}+r^{2}} z_{3} \overline{z_{3}}\right\}
$$

pode verifar-se que esta hipervariedade em $Q_{r}^{5}$ é invariante pela fibração de hopf. Assim sendo, podemos projetá-la em $\mathbb{C H}^{2}\left(\frac{4}{R^{2}}\right)$ e obter uma hipervariedade $S$ de $\mathbb{C H}^{2}\left(\frac{4}{R^{2}}\right)$

$$
S=\pi(\widetilde{S})=\left\{\left[\left(z_{1}, z_{2}, z_{3}\right)\right] \in \mathbb{C H}^{2}\left(\frac{4}{R^{2}}\right): \overline{z_{1}} z_{1}+\overline{z_{2}} z_{2}=r^{2} \text { e } \overline{z_{3}} z_{3}=R^{2}+r^{2}\right\} .
$$

Portanto, definindo $\tilde{f}: S_{r}^{3} \longrightarrow Q_{R}^{5}$ por

$$
\widetilde{f}\left(z_{1}, z_{2}\right)=\frac{1}{R}\left(z_{1}, z_{2}, \sqrt{R^{2}+r^{2}}\right)
$$

teremos uma imersão isométrica de $S_{r}^{3}$ em $Q_{R}^{5}$. Daí $f=\pi \circ \widetilde{f}$ nos dá uma imersão isométrica de $S_{r}^{3}$ em $\mathbb{C I H}^{2}\left(\frac{4}{R^{2}}\right)$.

Tomemos o referencial unitário do $\mathbb{C}^{3}$ restrito a $S$

$$
\begin{aligned}
\tilde{f}_{1} & =\left(-\bar{z}_{2}, \bar{z}_{1}, 0\right) \\
\tilde{f}_{2} & =\frac{\alpha}{r R}\left(z_{1}, z_{2}, \frac{r^{2}}{\alpha}\right) \\
\tilde{f}_{3} & =\frac{1}{R}\left(z_{1}, z_{2}, \alpha\right)
\end{aligned}
$$


sendo $\alpha$ uma constante dada por $\alpha=\sqrt{R^{2}+r^{2}}$. Sabemos que para o referencial $\left\{\tilde{f}_{1}, \tilde{f}_{2}, \widetilde{f}_{3}\right\}$ temos

$$
\begin{aligned}
& d \tilde{f}_{1}=\omega_{1}^{1} \otimes \tilde{f}_{1}+\omega_{1}^{2} \otimes \tilde{f}_{2}+\omega_{1}^{3} \otimes \tilde{f}_{3} \\
& d \tilde{f}_{2}=\omega_{2}^{1} \otimes \tilde{f}_{1}+\omega_{2}^{2} \otimes \tilde{f}_{2}+\omega_{2}^{3} \otimes \tilde{f}_{3} \\
& d \tilde{f}_{3}=\omega_{3}^{1} \otimes \tilde{f}_{1}+\omega_{3}^{2} \otimes \tilde{f}_{2}+\omega_{3}^{3} \otimes \tilde{f}_{3} .
\end{aligned}
$$

temos então usando os resultados da primeira seção a derivada covariante $\tilde{D}$ do $\mathbb{C H}^{2}\left(\frac{4}{R^{2}}\right)$

$$
\begin{aligned}
& \tilde{D} f_{1}=\left(\omega_{1}^{1}-\omega_{3}^{3}\right) \otimes f_{1}+\omega_{1}^{2} \otimes f_{2} \\
& \tilde{D} f_{2}=\omega_{2}^{1} \otimes f_{1}+\left(\omega_{2}^{2}-\omega_{3}^{3}\right) \otimes f_{2}
\end{aligned}
$$

restrito à quádrica $Q_{R}^{5}$ temos que $d \tilde{f}_{2}=\frac{\alpha}{r} d \tilde{f}_{3}$ e conseqüetemente

$$
\omega_{2}^{3}=\frac{\alpha}{r} \omega_{3}^{3} \quad \omega_{2}^{2}=\frac{\alpha}{r} \omega_{3}^{2} \quad \omega_{2}^{1}=\frac{\alpha}{r} \omega_{3}^{1} .
$$

Denotando por $\left\{\theta^{1} ; \theta^{2} ; \theta^{3}\right\}$ o referencial dual a $\left\{\tilde{f}_{1} ; \tilde{f}_{2} ; \tilde{f}_{3}\right\}$ e por $\left\{e_{1} ; e_{2} ; e_{3}\right\}$ a base canônica do $\mathbb{C}^{3}$ poderemos escrever

$$
\begin{aligned}
d \tilde{f}_{1} & =\frac{1}{r}\left(-d \overline{z_{2}} \otimes e_{1}+d \overline{z_{1}} \otimes e_{2}\right) \\
& =\left(\frac{\alpha}{r R} \overline{\theta^{2}}-\frac{1}{R} \overline{\theta^{3}}\right) \otimes f_{1}-\frac{\alpha}{r R} \overline{\theta^{1}} \otimes f_{2}-\frac{1}{R} \overline{\theta^{1}} \otimes f_{3} \\
d \tilde{f}_{2} & =\frac{\alpha}{r R}\left(d z_{1} \otimes e_{1}+d z_{2} \otimes e_{2}\right. \\
& =\left(\frac{\alpha}{r R} \theta^{1}\right) \otimes f_{1}+\left(\frac{\alpha^{3}}{r R^{3}} \theta^{2}-\frac{\alpha^{2}}{R^{3}} \theta^{3}\right) \otimes f_{2}-\left(\frac{\alpha^{2}}{R^{3}} \theta^{2}-\frac{r \alpha}{R^{3}} \theta^{3}\right) \otimes f_{3} \\
d \tilde{f}_{3} & =\frac{1}{R}\left(d z_{1} \otimes e_{1}+d z_{2} \otimes e_{2}\right. \\
& =\left(\frac{1}{R} \theta^{1}\right) \otimes f_{1}+\left(\frac{\alpha^{2}}{r R^{3}} \theta^{2}-\frac{r \alpha}{R^{3}} \theta^{3}\right) \otimes f_{2}-\left(\frac{r \alpha}{R^{3}} \theta^{2}-\frac{r^{2}}{R^{3}} \theta^{3}\right) \otimes f_{3} .
\end{aligned}
$$

Definindo $\theta=i\left(\frac{\alpha}{r R} \overline{\theta^{2}}-\frac{1}{R} \overline{\theta^{3}}\right)$, poderemos escrever para as formas de conexão

$$
\begin{array}{cc}
\omega_{1}^{1}=-i \theta & \omega_{2}^{1}=\frac{\alpha}{r R} \theta^{1} \\
\omega_{2}^{2}=-i \frac{\alpha^{2}}{R^{2}} \theta & \omega_{3}^{3}=i \frac{r^{2}}{R^{2}} \theta
\end{array}
$$


usando a igualdade acima teremos

$$
\begin{aligned}
& \tilde{D} f_{1}=i \frac{a f^{2}}{R^{2}} \theta \otimes f_{1}+\left(-\frac{a f}{r R} \overline{\theta^{1}}\right) \otimes f_{2} \\
& \tilde{D} f_{2}=i \frac{a f}{R} \theta^{1} \otimes f_{1}+(-i \theta) \otimes f_{2}
\end{aligned}
$$

$\mathcal{D}$ será dada por $\mathcal{D}=\left\{\mu f_{1}: \mu \in \mathbb{C}\right\}$. Daí, por analogia ao caso da imersão da esfera de raio $r$ em $\mathbb{C P}^{2}\left(\frac{4}{R^{2}}\right)$, faremos $\eta=f_{2}$ e $\xi=-J\left(f_{2}\right)$

$$
\begin{aligned}
\tilde{D} \eta & =\frac{\alpha}{r R} \theta^{1} \otimes f_{1}+\theta \otimes \xi \\
\tilde{D} \xi & =-\frac{\alpha}{r R} \theta^{1} \otimes\left(i f_{1}\right)+(-\theta) \otimes \eta
\end{aligned}
$$

e disto tiramos que $D \xi=-\frac{\alpha}{r R} \theta^{1} \otimes\left(i f_{1}\right)$. Temos assim tudo que necessitamos para calcular a representação matricial de $D \xi$

$$
\begin{aligned}
D_{f_{1}} \xi & =-\frac{\alpha}{r R}\left(i f_{1}\right) \\
D_{i f_{1}} \xi & =\frac{\alpha}{r R} f_{1}
\end{aligned}
$$

e daí concluímos que $\tau=0$ e

$$
h=\left[\begin{array}{cc}
0 & \frac{\alpha}{r R} \\
-\frac{\alpha}{r R} & 0
\end{array}\right]
$$

Como $\operatorname{tr}(B)=b(\xi, \xi)=-\left\langle\tilde{D}_{\xi} \eta, \xi\right\rangle=-\theta(\xi)=\frac{\alpha}{R} \neq 0$ outra imersão da esfera $S_{r}^{3}$ em $\mathbb{C} \mathbb{H}^{2}\left(\frac{4}{R^{2}}\right)$ diferirá desta por uma isometria de $\mathbb{C} \mathbb{H}^{2}\left(\frac{4}{R^{2}}\right)$.

\subsection{Imersão isométrica de $Q_{r}^{3}$ em $N(c)$}

Imersão de $Q_{r}^{3}$ em $\mathbb{C H}^{2}\left(\frac{4}{R^{2}}\right)$ :

A imersão da quádrica $Q_{r}^{3}$ pode ser obtida da seguinte forma: Sabemos que

$$
T_{z} Q_{r}^{5}=\left\{v \in \mathbb{C}^{3}: \operatorname{Reb}(z, v)=0\right\}
$$

e que a distribuição $\widetilde{\mathcal{D}}$ é obtida tomando o núcleo da 1- forma complexa

$$
\omega_{z}=-\bar{z}_{0} d z_{0}+\bar{z}_{1} d z_{1}+\bar{z}_{2} d z_{2}
$$

Seja $\widetilde{S}$ a hipervariedade de $Q_{R}^{5}$ definida por

$$
\widetilde{S}=\left\{\left(z_{0}, z_{1}, z_{2}\right) \in Q_{R}^{5}:-\overline{z_{0}} z_{0}+\overline{z_{1}} z_{1}=\frac{r^{2}}{R^{2}-r^{2}} \overline{z_{2}} z_{2}\right\}
$$


podemos projetar, por motivos já explicados, $\widetilde{S}$ em $\mathbb{C H}^{2}\left(\frac{4}{R^{2}}\right)$ e obter uma hipervariedade $S$ de $\mathbb{C H}^{2}\left(\frac{4}{R^{2}}\right)$

$$
S=\pi(\widetilde{S})=\left\{\left[\left(z_{0}, z_{1}, z_{2}\right)\right] \in \mathbb{C H}^{2}\left(\frac{4}{R^{2}}\right): \overline{z_{0}} z_{0}+\overline{z_{1}} z_{1}=r^{2} e \overline{z_{2}} z_{2}=R^{2}-r^{2}\right\} .
$$

Portanto, definindo $\tilde{f}: Q_{r}^{3} \longrightarrow Q_{R}^{5}$ por

$$
\tilde{f}\left(z_{1}, z_{2}\right)=\frac{1}{R}\left(z_{0}, z_{1}, \sqrt{R^{2}-r^{2}}\right)
$$

teremos uma imersão isométrica de $Q_{r}^{3}$ em $Q_{R}^{5}$. Daí $f=\pi \circ \tilde{f}$ nos dá uma imersão isométrica de $Q_{r}^{3}$ em $\mathbb{C H}^{2}\left(\frac{4}{R^{2}}\right)$.

Começaremos o cálculo de $\tau, h$ e $b$ para esta imersão. Consideremos inicialmente o seguinte referencial unitário do $\mathbb{C}^{3}$ restrito a $S$

$$
\begin{aligned}
\tilde{f}_{0} & =\frac{1}{R}\left(z_{0}, z_{1}, \alpha\right) \\
\tilde{f}_{1} & =\frac{1}{r}\left(\overline{z_{1}}, \bar{z}_{0}, 0\right) \\
\tilde{f}_{2} & =\frac{\alpha}{r R}\left(z_{0}, z_{1}, \frac{r^{2}}{\alpha}\right)
\end{aligned}
$$

sendo $\alpha$ uma constante dada por $\alpha=\sqrt{r^{2}-R^{2}}$. Sabemos que o referencial $\left\{\widetilde{f}_{1}, \widetilde{f}_{2}, \widetilde{f}_{3}\right\}$ pode ser estendido a um aberto de $\mathbb{C}^{3}$. Neste caso temos

$$
\begin{aligned}
& d \tilde{f}_{0}=\omega_{0}^{0} \otimes \tilde{f}_{0}+\omega_{0}^{1} \otimes \tilde{f}_{2}+\omega_{0}^{2} \otimes \tilde{f}_{2} \\
& d \tilde{f}_{1}=\omega_{1}^{0} \otimes \tilde{f}_{0}+\omega_{1}^{1} \otimes \tilde{f}_{1}+\omega_{1}^{2} \otimes \tilde{f}_{1} \\
& d \tilde{f}_{2}=\omega_{2}^{0} \otimes \tilde{f}_{0}+\omega_{2}^{1} \otimes \tilde{f}_{1}+\omega_{2}^{2} \otimes \tilde{f}_{1}
\end{aligned}
$$

temos então usando os resultados da primeira seção a derivada covariante $\tilde{D}$ do $\mathbb{C H}^{2}\left(\frac{4}{R^{2}}\right)$

$$
\begin{aligned}
& \tilde{D} f_{1}=\left(\omega_{1}^{1}-\omega_{3}^{3}\right) \otimes f_{1}+\omega_{1}^{2} \otimes f_{2} \\
& \tilde{D} f_{2}=\omega_{2}^{1} \otimes f_{1}+\left(\omega_{2}^{2}-\omega_{3}^{3}\right) \otimes f_{2}
\end{aligned}
$$

restrito à quádrica $Q_{R}^{5}$ temos que $d \tilde{f}_{2}=\frac{\alpha}{r} d \tilde{f}_{0}$ e conseqüetemente

$$
\omega_{2}^{0}=\frac{\alpha}{r} \omega_{0}^{0}, \quad \omega_{2}^{2}=\frac{\alpha}{r} \omega_{0}^{2}, \quad \omega_{2}^{1}=\frac{\alpha}{r} \omega_{0}^{1} .
$$

Denotando por $\left\{\theta^{1} ; \theta^{2} ; \theta^{3}\right\}$ o referencial dual a $\left\{\tilde{f}_{1} ; \tilde{f}_{2} ; \tilde{f}_{3}\right\}$ e por $\left\{e_{1} ; e_{2} ; e_{3}\right\}$ a base canônica do $\mathbb{C}^{3}$ poderemos escrever

$$
d \tilde{f}_{0}=\frac{1}{R}\left(d z_{0} \otimes e_{0}+d z_{1} \otimes e_{1}\right)
$$




$$
\begin{aligned}
& =\left(\frac{r \alpha}{R^{3}} \theta^{1}+\frac{r^{2}}{R^{3}} \theta^{0}\right) \otimes f_{0}+\left(\frac{1}{R} \theta^{0}\right) \otimes f_{1}+\left(\frac{\alpha^{2}}{r R^{3}} \theta^{2}+\frac{r \alpha}{R^{3}} \theta^{0}\right) \otimes f_{2} \\
d \tilde{f}_{1} & =\frac{1}{r}\left(d \bar{z}_{1} \otimes e_{1}+d \bar{z}_{0} \otimes e_{2}\right) \\
& =\frac{1}{R} \bar{\theta}^{1} \otimes f_{0}\left(\frac{\alpha}{r R} \bar{\theta}^{1}+\frac{1}{R} \bar{\theta}^{0}\right) \otimes f_{1}-\frac{\alpha}{r R} \bar{\theta}^{1} \otimes f_{2} \\
d \tilde{f}_{2} & =\frac{\alpha}{r R}\left(d z_{1} \otimes e_{1}+d z_{2} \otimes e_{2}\right) \\
& =\left(\frac{a f^{2}}{R^{3}} \theta^{2}+\frac{r \alpha}{R^{3}} \theta^{3}\right) \otimes f_{0}+\left(\frac{\alpha}{r R} \theta^{1}\right) \otimes f_{1}+\left(\frac{\alpha^{3}}{r R^{3}} \theta^{2}+\frac{\alpha^{2}}{R^{3}} \theta^{0}\right) \otimes f_{2} .
\end{aligned}
$$

Definindo $\theta=i\left(\frac{\alpha}{r R} \theta^{1}+\frac{1}{R} \theta^{0}\right)$ poderemos escrever para as formas de conexão

$$
\begin{array}{cc}
\omega_{1}^{1}=-i \theta & \omega_{1}^{2}=\frac{\alpha}{r R} \theta^{1} \\
\omega_{2}^{2}=-i \frac{\alpha^{2}}{R^{2}} \theta & \omega_{0}^{0}=i \frac{r^{2}}{R^{2}} \theta
\end{array}
$$

e logo temos

$$
\begin{aligned}
& \tilde{D} f_{1}=i \frac{\alpha^{2}}{R^{2}} \theta \otimes f_{1}+\left(-\frac{\alpha}{r R} \overline{\theta^{1}}\right) \otimes f_{2} \\
& \tilde{D} f_{2}=i \frac{\alpha}{R} \theta^{1} \otimes f_{1}+(-i \theta) \otimes f_{2} .
\end{aligned}
$$

A distribuição holomorfa $\mathcal{D}$ desta imersão da qüádrica é dada por $\mathcal{D}=\left\{\mu f_{1}: \mu \in \mathbb{C}\right\}$. Daí voltando a notação $\eta=f_{2}$ e $\xi=-J\left(f_{2}\right)$

$$
\begin{aligned}
\tilde{D} \eta & =\frac{\alpha}{r R} \theta^{1} \otimes f_{1}+\theta \otimes \xi \\
\tilde{D} \xi & =-\frac{\alpha}{r R} \theta^{1} \otimes\left(i f_{1}\right)+(-\theta) \otimes \eta
\end{aligned}
$$

e disto tiramos que $D \xi=-\frac{\alpha}{r R} \theta^{1} \otimes\left(i f_{1}\right)$. Temos assim tudo que necessitamos para calcular a representação matricial de $D \xi$

$$
\begin{aligned}
D_{f_{1}} \xi & =-\frac{\alpha}{r R}\left(i f_{1}\right) \\
D_{i f_{1}} \xi & =\frac{\alpha}{r R} f_{1}
\end{aligned}
$$

e daí concluímos que $\tau=0 \mathrm{e}$

$$
h=\left[\begin{array}{cc}
0 & -\frac{\alpha}{r R} \\
\frac{\alpha}{r R} & 0
\end{array}\right] .
$$


Além disso $b(\xi, \xi)=-\left\langle\tilde{D}_{\xi} \eta, \xi\right\rangle=-\theta(\xi)=\frac{\alpha}{R} \neq 0$. Temos então pelo teorema anterior que qualque outra imersão da esfera $S_{r}^{3} \operatorname{em~} \mathbb{C P}^{2}\left(\frac{4}{R^{2}}\right)$ tal que $b(\xi, \xi)$ for não nulo diferir desta imersão por uma isometria de $\mathbb{C P}^{2}\left(\frac{4}{R^{2}}\right)$.

\subsection{Imersão isométrica de $H^{3}$ em $N(c)$}

Imersão de $H^{3}$ em $\mathbb{C H}^{2}\left(\frac{4}{R^{2}}\right)$ :

Fixada uma base $\left\{f_{0} ; f_{1} ; f_{2}\right\}$ de $\mathbb{C}^{3}$ sejam $b: \mathbb{C}^{3} \times \mathbb{C}^{3} \longrightarrow \mathbb{C}^{3}$ a forma sesquilinear dada por

$$
b(z, w)=z_{1} \bar{w}_{1}+i\left(z_{0} \bar{w}_{2}-z_{2} \bar{w}_{0}\right)
$$

e $S U(2,1)=\left\{T: \mathbb{C}^{3} \longrightarrow \mathbb{C}^{3}: T\right.$ é $\mathbb{C}$-linear, $\operatorname{det}(T)=1$ e $b(T z, T w)=b(z, w)$ para todos $\left.z, w \in \mathbb{C}^{3}\right\}$. Em $S U(2,1)$ o sub-grupo $G$ definido por

$$
G=\left\{T \in S U(2,1): T\left(f_{1}\right)=f_{1}\right\}
$$

Em $\mathbb{C}^{3}$ definamos

$$
H^{3}=\left\{\quad z=\left(1, z_{1}, z_{2}\right) \in \mathbb{C}^{3}: b(z, z)=0 \quad\right\}
$$

$H^{3}$ é uma subvariedade de $\mathbb{C}^{2}$. A distribuição holomorfa de $H^{3}$ é dada pelo espaço complexo gerado por $\frac{\partial}{\partial z_{1}}-i \bar{z}_{1} \frac{\partial}{\partial z_{2}}$. O grupo $G$ age em $H^{3}$ da seguinte forma

$$
\begin{aligned}
G \times H^{3} & \longrightarrow H^{3} \\
(T, z) & \longrightarrow T z .
\end{aligned}
$$

De resultados anteriores sabemos que

$$
G=\left\{\left(\begin{array}{ccc}
1 & 0 & 0 \\
\frac{z}{\sqrt{2}} & 1 & 0 \\
x+\frac{i}{2}|z|^{2} & i \frac{\bar{z}}{\sqrt{2}} & 1
\end{array}\right): \in \mathbb{C}, x \in \mathbb{R}\right\}
$$

e que $H^{3} \cong G$. Mudando abase $f=\left\{f_{0} ; f_{1} ; f_{2}\right\}$ para a base $e$ dada por $e_{0}=\frac{f_{0}+i f_{2}}{\sqrt{2}}$, $e_{1}=f_{1}$ e $e_{2}=\frac{i f_{0}+f_{2}}{\sqrt{2}}$, então o grupo $G$ se escreve na nova base como

$$
G=\left\{\left(\begin{array}{ccc}
\frac{1}{\sqrt{2}} & 0 & \frac{-i}{\sqrt{2}} \\
0 & 1 & 0 \\
\frac{-i}{\sqrt{2}} & 0 & \frac{1}{\sqrt{2}}
\end{array}\right)\left(\begin{array}{ccc}
1 & 0 & 0 \\
\frac{z}{\sqrt{2}} & 1 & 0 \\
x+\frac{i}{2}|z|^{2} & i \frac{\bar{z}}{\sqrt{2}} & 1
\end{array}\right)\left(\begin{array}{ccc}
\frac{1}{\sqrt{2}} & 0 & \frac{i}{\sqrt{2}} \\
0 & 1 & 0 \\
\frac{i}{\sqrt{2}} & 0 & \frac{1}{\sqrt{2}}
\end{array}\right): z \in \mathbb{C} \quad \text { e } \quad x \in \mathbb{R}\right\}
$$




$$
G=\left\{\left(\begin{array}{ccc}
1+\frac{|z|^{2}}{4}-\frac{i}{2} x & \frac{1}{2} \bar{z} & \frac{1}{2}\left(x+\frac{i}{2}|z|^{2}\right) \\
\frac{z}{2} & 1 & \frac{i}{2} z \\
\frac{1}{2}\left(x+\frac{i}{2}|z|^{2}\right) & \frac{i}{2} \bar{z} & 1-\frac{1}{4}|z|^{2}+\frac{i}{2} x
\end{array}\right): z \in \mathbb{C} \quad \text { e } \quad x \in \mathbb{R}\right\}
$$

Pela forma como chegamos ao grupo $G$ é imediato que ele deixa invariante o hiperboloide complexo $Q_{r}^{5}=\left\{z_{o} e_{0}+z_{1} e_{1}+z_{2} e_{2}:-z_{0} \bar{z}_{0}+z_{1} \bar{z}_{1}+z_{2} \bar{z}_{2}=-r^{2}\right\}$. De fato, é suficiente verificar que $-z_{0} \bar{z}_{0}+z_{1} \bar{z}_{1}+z_{2} \bar{z}_{2}$ e $z_{1} \bar{z}_{1}+i\left(z_{0} \bar{z}_{2}-z_{2} \bar{z}_{0}\right)$ diferem apenas pela mudança de base feita acima. Como $r e_{0} \in Q_{r}^{5}$ sua órbita $\mathcal{O}\left(r e_{0}\right)$ pela ação de $G$ estará contida em $Q_{r}^{5}$

$$
\begin{aligned}
& \mathcal{O}\left(r e_{0}\right)=\left\{r T\left(e_{0}\right): \quad T \in G\right\} \\
& \mathcal{O}\left(r e_{0}\right)=\left\{r\left(1+\frac{|z|^{2}}{4}-\frac{i}{2} x, \frac{z}{2}, \frac{1}{2}\left(x+\frac{i}{2}|z|^{2}\right)\right) \in \mathbb{C}^{3} \quad: \quad z \in \mathbb{C}, \quad x \in \mathbb{R}\right\} .
\end{aligned}
$$

Uma imersão de $f$ de $H^{3}$ em $\mathbb{C H}^{2}$, usando a idetificação de $\mathbb{C H}^{2}$ com o disco $D^{2}$, é dada por

$$
\begin{gathered}
f: H^{3} \longrightarrow D^{2} \\
f(z, x)=r\left(\frac{2 z}{4+|z|^{2}-2 i x}, \frac{2\left(x+i|z|^{2}\right)}{4+|z|^{2}-2 i x}\right) .
\end{gathered}
$$




\section{Referências Bibliográficas}

[1] M. F. Atiyah, I. G. Macdonald, Introduction to Commutative Algebra, AddisonWesley, 1969.

[2] M. Diniz, Variedades sub-Riemannianas de contato de dimensão 3, Dissertação de mestrado IME-USP, 1996.

[3] M. P. do Carmo, Geometria Riemanniana, Projeto Euclides, IMPA, Rio de Janeiro, 1988.

[4] E. Falbel, J. A. Verderesi and J. M. Veloso, The equivalence problem in subRiemannian geomentry, Relatório Técnico IME-USP, 1993.

[5] E. Falbel, C. Gorodski, Sub-Riemannian Homoge neous Spaces in dimensions 3 and 4. Geometria Dedicata 63, Vol. 3, 227-252, 1996.

[6] S. Helgason, Differential Geometry and Symmetric Spaces, Academic Press, New York, 1963.

[7] G. R. Jensen, Higher Order Contact of Submanifolds of Homogeneous spaces, Lecture Notes in Math., 610, Springer-Verlarg, New-York, 1977.

[8] J. Milnor, Curvatures of Left Invariant Metrics On Lie Groups, Advances in Math., Vol. 21, 293-329, 1976.

[9] J. C. S. de Miranda., Primeira Variação da Energia e Geodésicas na Geometria SubRiemanniana, Dissertação de Mestrado IME-USP, 1998.

[10] M. Okomura, On some real hypersurfaces of a complex projective space, Transactions of American Math. Soc., Vol. 212, 355-364, 1975. 
[11] P. J. Ryan, Homogeneity and some curvature conditions for hypersurfaces, Tôhoku Math. Journal, Vol. 21, num. 2, 363-388, 1969.

[12] B. Smith, Differential of complex hipersurfaces, Annals of Math., 85, 247-266, 1975.

[13] R. Strichartz, Sub-Riemannian Geometry, Journal of differential geometry, 24 (1986), 221-263.

[14] Y. J. Suh and R. Takagi, A rigidity for real hypersurfaces in a complex projective space, Tôhoku Math. Journal 43 (1991), 501-507.

[15] R. Takagi, On homogeneous real hypersurfaces in a complex projective space, Osaka Journal Math., Vol. 10, 495-507, 1973.

[16] R. Takagi,Real hipersurfaces in a complex projective space with constant principal curvatures, J. Math Soc Japan, 27(1975), 43-53.

[17] R. Takagi,Real hipersurfaces in a complex projective space with constant principal curvatures II, J. Math Soc Japan, 27(1975).

[18] R. Takagi, I. Kim and B. H. Kim, The Rigidity for Real Hypersurfaces in a Complex Projective Spaces, Tôhoku Math. Journal 50 (1998), 532-536.

[19] J. A. Verderesi, Tese de Livre Docência, IME-USP, 1998.

[20] J. A. Verderesi, Variedades sub-Riemannianas em $M^{2}(c)$, Notas relativas à Tese de Livre-docência, IME-USP, 1998.

[21] A. M. Vershik, Y. Ya. Gershkovich, Non-holonomic Dynamical Systems, Geometry of Distributions and Variational Problems, em: V. I. Arnol'd, S. P. Novikov Eds., Dynamical Systems VII, Encyclopedia Mathematical, Sciences Series, Vol. 16, Springer-Verlag, NY, 1994.

[22] K. Yano and M. Kon, CR-submanifolds of Kaehlerian and Sasakian Manifolds, Progress in Math., Vol. 30, Birkhauser, 1983. 